\title{
New genus and new species of spittlebugs (Hemiptera: Cercopidae) from the Philippines
}

\author{
Elorde Jr. S. CRISPOLON (i) 1,*, Eric GUILBERT (i) 2, \\ Sheryl A. YAP ${ }^{3}{ }^{3}$ \& Adeline SOULIER-PERKINS ${ }^{4}{ }^{4}$ \\ 1,2,4 Mécanismes adaptatifs et évolution (MECADEV), Muséum national d'Histoire naturelle, \\ CNRS; CP 50, Entomologie, 57 rue Cuvier, 75005 Paris, France. \\ ${ }^{1}$ Department of Entomology, College of Agriculture, University of Southern Mindanao, \\ Kabacan Cotabato, Philippines. \\ ${ }^{3}$ Institute of Weed Science, Entomology and Plant Pathology, College of Agriculture and Food \\ Science, University of the Philippines Los Baños, College, Laguna, 4031, Philippines \\ and Museum of Natural History, University of the Philippines Los Baños, \\ Laguna, 4031, Philippines. \\ *Corresponding author: elorde.crispolon@edu.mnhn.fr \\ ${ }^{2}$ Email: eric.guilbert@mnhn.fr \\ 32Email: sayap3@up.edu.ph \\ ${ }^{4}$ Email: adeline.soulier@mnhn.fr

\footnotetext{
${ }^{1}$ urn:lsid:zoobank.org:author:9FAB1 A02-BAF4-4E94-8C5D-2A9D6E4D95BD

${ }^{2}$ urn:lsid:zoobank.org:author:A221ADFB-788C-475F-90A6-FFB673931AF6

${ }^{3}$ urn:lsid:zoobank.org:author:8347CEBE-442B-4583-BE00-B4BF0502FFEA

${ }^{4}$ urn:lsid:zoobank.org:author:780D689B-DAD3-4115-9C34-97C27B580EB9
}

\begin{abstract}
The following new taxa are described from the Philippines: Mioscarta nubisa Crispolon \& Soulier-Perkins sp. nov., M. translucida Crispolon \& Yap sp. nov. and Trigonoschema Crispolon \& Soulier-Perkins gen. nov. with three new species: T. manoborum Crispolon \& Soulier-Perkins sp. nov. (as type species), T. negrosensis Crispolon \& Yap sp. nov and T. rubercella Crispolon \& Guilbert sp. nov. Trigonoschema pallida (Lallemand, 1927) comb. nov. is transferred from Mioscarta Breddin, 1901. Descriptions of male genitalia are illustrated and keys to species of Philippine Mioscarta and Trigonoschema gen. nov. are provided. Although phylogenetic results confirm the monophyly of all genera and Trigonoschema being a distinct genus from Mioscarta, relationships between genera remain uncertain. A checklist of the genera and species of Cercopidae found in the Philippines is included.
\end{abstract}

Key words. Auchenorrhyncha, Rhinaulacini, froghopper, Cicadomorpha, molecular phylogeny.

Crispolon E. Jr. S., Guilbert E., Yap S.A. \& Soulier-Perkins A. 2021. New genus and new species of spittlebugs (Hemiptera: Cercopidae) from the Philippines. European Journal of Taxonomy 778: 90-135.

https://doi.org/10.5852/ejt.2021.778.1571 


\section{Introduction}

Currently 18 genera of Cercopidae are known from the Philippines with 65 species and 9 subspecies (Table 1). Three genera and most of these species are endemic to the Philippines and are generally recorded throughout its three main geographical regions: Luzon, Visayas and Mindanao. Thirteen species cannot be precisely located on the archipelago since no exact locations were provided in the original descriptions (Soulier-Perkins 2020). Luzon constitutes a distinct centre of endemism (Vallejo 2014), which could explain the high number of Cercopidae found on the island. The Visayas is composed of many islands and is located in the central part of the archipelago. Out of the 65 species known, only six are recorded from the Visayan Islands, three in Samar, two in Negros and one in Panay. Of the three main islands, Mindanao is said to be the least explored (Crispolon et al. 2019), this is most likely why so few cercopids are recorded from it. With a more extensive exploration especially in the central and south geographical parts of the archipelago, we expect to discover more species. Some recent explorations on the main island Luzon, Negros and Mindanao, led us to find two new species for Mioscarta Breddin, 1901 and a new genus Trigonoschema Crispolon \& Soulier-Perkins gen. nov. for which three species are described here. This brings to a total of four genera endemic to the Philippines and 70 for the number of cercopid species known for this archipelago (Table 1) with 87\% endemicity (Soulier-Perkins 2020).

In order to ease the identification of Mioscarta and Trigonoschema gen. nov. species present in the Philippines, keys are provided and if possible the male terminalia are described and illustrated. Since we hypothesized that Trigonoschema gen. nov is sufficiently morphologically distinct from Mioscarta, to be described as a new genus, we tested the species for which we had molecular samples using molecular characters in Bayesian inference and Maximum likelihood reconstruction analyses. Lallemand (1949) and Lallemand \& Synave (1961) provided a generic key for the family, which remains the only complete key available. Interactive keys, for the Cercopidae genera found in the Philippines and for the Poeciloterpa Stål, 1870 species can be accessed throughout the web page dedicated to keys (https://hemiptera-databases.org/cool/id_keys.php) in the database Cercopoidea Organized On Line (COOL). A key to the species of Poeciloterpa is also available in Crispolon et al. (2019).

Cercopids are generally not confined to one host and not bound to oligophagy (Soulier-Perkins \& Le Cesne 2016). They feed on diverse host-plants such as pastures crops, sugarcane, eucalyptus, rice and maize in Africa, America (Mello et al. 1996; Holmann \& Peck 2002; Thompson 2004; Carvalho \& Webb 2005; Paladini et al. 2008; Bartlett et al. 2018; Thompson et al. 2020), China and Southeast Asia (Chen \& Liang 2015, Su et al. 2018) and they are also associated with nitrogen-fixing angiosperm (Thompson 2004; Deitz et al. 2008). In the Philippines, Crispolon et al. (2019) have recorded new hostplants associated to species of Poeciloterpa. However, collectors often used light traps so, very little information on the natural history is available for the species of Mioscarta and Trigonoschema gen. nov. and their host plants, biology and other ecological data remain very limited.

\section{Material and Methods}

\section{Taxonomic study}

\section{Preparation of specimen for morphological observation}

For type and other material examined, when label citations cannot be reliably interpreted and formatted they are cited verbatim in double quotation marks: "”. When the label is interpreted the data is placed in square brackets: [ ] e.g., coordinates interpreted from a locality, or translations of foreign text.

The abdomen of each specimen examined was cut off and cleared for 20 minutes in hot $\left(85^{\circ} \mathrm{C}\right) 10 \%$ $\mathrm{KOH}$. Dissections and cleaning of genital structures were performed in distilled water. If needed, a few drops of blue paragon for dying the ectodermic genital ducts were added for a few minutes. Observations were done in glycerol using a Leica MZ16 stereo microscope on which a camera lucida is attached in 
Table 1. Checklist of genera, species and subspecies found in the Philippines.

\begin{tabular}{|c|c|c|}
\hline Genera & Species and subspecies & Philippine Islands \\
\hline \multirow[t]{7}{*}{ Cosmoscarta Stål, 1869} & C. consociata Distant, 1900 & Main Island Luzon \\
\hline & C. lateralis Jacobi, 1927 & Samar Island \\
\hline & C. nigroguttata Stål, 1870 & Philippine Islands \\
\hline & C. semimaculata Stål, 1870 & Philippine Islands \\
\hline & C. sexmaculata Stål, 1870 & Philippine Islands \\
\hline & C. sulukensis Distant, 1900 & Sulu Islands \\
\hline & C. whiteheadi Distant, 1900 & Main Island Luzon \\
\hline Ectemnonotum Schmidt, 1909 & E. luzonensis (Lallemand, 1931) & Luzon Islands \\
\hline \multirow[t]{2}{*}{ Eoscarta Breddin, 1902} & E. borealis (Distant, 1878) & Main Island Mindanao \\
\hline & E. philippinica Lallemand, 1949 & Main Island Mindanao \\
\hline Euglobiceps Lallemand, 1923 & E. elongata Lallemand, 1923 & Main Island Mindanao \\
\hline \multirow[t]{11}{*}{ Gynopygoplax Schmidt 1909} & G. bicolor Lallemand, 1956 & Mindoro Island \\
\hline & G. daphne (Stål, 1865) & Luzon Islands \\
\hline & G. inclusa Lallemand, 1922 & Balabac Island \\
\hline & G. inclusiformis Schmidt, 1911 & Palawan Island \\
\hline & G. luzonensis Schmidt, 1909 & Luzon Islands \\
\hline & G. meyeri Schmidt, 1909 & Philippine Islands \\
\hline & G. mounseyi Lallemand, 1927 & Palawan Island \\
\hline & G. proserpina (White, 1845) & Luzon Islands \\
\hline & G. proserpinella Schmidt, 1909 & Luzon Islands \\
\hline & G. theora (White, 1845) & Philippine Islands \\
\hline & G. walkeri Metcalf, 1961 & Luzon Islands \\
\hline \multirow[t]{3}{*}{ Homalostethus Schmidt, 1910} & H. dirce (Breddin, 1901) & Sulu Islands \\
\hline & H. sangaris (Jacobi, 1905) & Luzon Islands \\
\hline & H. spectabilis (Burmeister, 1834) & Luzon Islands \\
\hline Jacobsoniella Melichar, 1914 & J. bakeri Schmidt 1920 & Luzon Islands \\
\hline \multirow[t]{8}{*}{ Leptataspis Schmidt, 1910} & L. bukidnona Lallemand 1923 & Main Island Mindanao \\
\hline & L. butuanensis Lallemand 1923 & Main Island Mindanao \\
\hline & L. insularis Lallemand 1927 & Philippine Islands \\
\hline & L. palawana Schmidt, 1911 & Palawan Island \\
\hline & L. philippinensis Schmidt, 1920 & Luzon Islands \\
\hline & L. proserpinopsis Schmidt, 1911 & Luzon Islands \\
\hline & L. rotundata (Walker, 1858) & Philippine Islands \\
\hline & L. rufipes Stål, 1870 & Philippine Islands \\
\hline \multirow[t]{8}{*}{ Mioscarta Breddin, 1901} & M. basilana Jacobi, 1927 & Basilan Island \\
\hline & M. ferruginea (Walker, 1851) & Samar Island \\
\hline & M. flavosalis Jacobi, 1927 & Samar Island \\
\hline & M. lutea Schmidt, 1925 & Luzon Islands \\
\hline & M. nubisa Crispolon \& Soulier-Perkins sp & Main Island Luzon \\
\hline & M. obscuripennis Schmidt, 1920 & Main Island Luzon, Negros Island \\
\hline & M. semperi Jacobi, 1905 & Luzon Islands, Mindanao Islands \\
\hline & M. translucida Crispolon \& Yap sp. nov. & Main Island Luzon \\
\hline $\begin{array}{l}\text { Opistharsostethus Schmidt, } \\
1911\end{array}$ & O. calypso Lallemand, 1923 & Polillo Island \\
\hline \multirow[t]{2}{*}{ Oxymegaspis Schmidt, 1911} & O. izzardi Lallemand, 1954 & Luzon Islands \\
\hline & O. schultzei (Schmidt, 1931) & Luzon Islands \\
\hline
\end{tabular}


CRISPOLON E. Jr. S. et al., New genus and new species of spittlebugs from the Philippines

\begin{tabular}{|c|c|c|}
\hline \multicolumn{2}{|c|}{$\begin{array}{l}\text { Paratrichoscarta Lallemand \& P. carinata (Stål, 1870) } \\
\text { Synave, } 1953\end{array}$} & Philippine Islands \\
\hline \multirow[t]{11}{*}{ Phymatostetha Stål, 1870} & P. cincta Lallemand, 1923 & Balabac Island, Palawan Island \\
\hline & P. perspicillaris (White, 1845) & Philippine Islands \\
\hline & -P.p. dapitana Lallemand 1923 & $\begin{array}{l}\text { Basilan Island, Main Island } \\
\text { Mindanao }\end{array}$ \\
\hline & -P. p. extensa Lallemand, 1951 & Mindanao Islands \\
\hline & -P.p. flavopicta Distant, 1900 & Luzon Islands \\
\hline & -P. p. iligana Lallemand, 1923 & Main Island Mindanao \\
\hline & -P.p. mactans White, 1845 & Luzon Islands \\
\hline & - P. p. montana Schmidt, 1920 & Main Island Luzon \\
\hline & - P. p. rubens Lallemand, 1923 & Main Island Luzon \\
\hline & - P. p. sibuyana Lallemand, 1951 & Sibuyan Island \\
\hline & - P. p. variegata Lallemand, 1951 & Mindanao Islands \\
\hline \multirow[t]{13}{*}{ Poeciloterpa Stål 1870} & $\begin{array}{l}\text { P. altissima Crispolon \& Soulier-Perkins, } \\
2019\end{array}$ & Main Island Mindanao \\
\hline & P. atra Jacobi, 1927 & Polillo Island, Main Island Luzon \\
\hline & P. conica Crispolon \& Soulier-Perkins, 2019 & Negros Island \\
\hline & P. fusca Lallemand 1927 & Philippine Islands \\
\hline & P. gapudi Crispolon \& Yap, 2019 & Main Island Mindanao \\
\hline & P. latipennis Schmidt, 1920 & $\begin{array}{l}\text { Main Island Luzon, Main Island } \\
\text { Mindanao }\end{array}$ \\
\hline & P. mangkas Crispolon \& Yap, 2019 & Negros Island \\
\hline & P. minuta Lallemand, 1922 & $\begin{array}{l}\text { Main Island Luzon, Siargao } \\
\text { Island }\end{array}$ \\
\hline & P. montana Schmidt, 1927 & Main Island Luzon \\
\hline & P. nigrolimbata Stål 1870 & Main Island Luzon \\
\hline & P. obscura Schmidt, 1927 & $\begin{array}{l}\text { Main Island Luzon, Main Island } \\
\text { Mindanao }\end{array}$ \\
\hline & P. rufolimbata Schmidt, 1927 & Panay Island \\
\hline & P. unicolor Lallemand, 1922 & Main Island Luzon \\
\hline Radioscarta Lallemand, 1923 & R. surigaona Lallemand, 1923 & Main Island Mindanao \\
\hline \multirow[t]{2}{*}{ Serapita Schmidt, 1909} & S. charon (White, 1845 ) & Philippine Islands \\
\hline & S. phillippinensis Lallemand,1922 & Main Island Luzon \\
\hline Suracarta Schmidt, 1909 & S. torquata (Jacobi 1905) & Palawan Island \\
\hline Trichoscarta Breddin, 1902 & T. luteomaculata Lallemand 1922 & Palawan Island \\
\hline \multirow[t]{4}{*}{$\begin{array}{l}\text { Trigonoschema Crispolon \& } \\
\text { Soulier-Perkins gen. nov. }\end{array}$} & $\begin{array}{l}\text { T. manoborum Crispolon \& Soulier- } \\
\text { Perkins sp. nov. }\end{array}$ & Main Island Mindanao \\
\hline & T. negrosensis Crispolon \& Yap sp. nov. & Negros Island, Main Island Luzon \\
\hline & T. pallida (Lallemand, 1927) comb. nov. & Philippine Islands \\
\hline & T. rubercella Crispolon \& Guilbert sp. nov & Negros Island \\
\hline
\end{tabular}

order to draw the terminalia. The photos of the habitus were taken using a stereo microscope Leica MZ16 with IC3D digital camera; final images were produced using Helicon 5.0 software. Schematic illustration of the head in frontal view (Fig. 1) for ocelli size and distance comparison and lateral view of the insect body for the pronotum angle (Fig. 2) were also done. Posterior wings of Mioscarta and Poeciloterpa were also illustrated (Fig. 3). Terminalia terminologies follow Soulier-Perkins \& Kunz (2012) and Le Cesne et al. (2021). Specifically, the sterno-lateral plate often referred as the lateral plate in the previous literature. For each newly described species, two authors are selected for the authorship: the first author followed alternatively by one of the three other authors. 


\section{Molecular study}

\section{Taxon and data sampling}

A phylogenetic analysis based on molecular characters is performed in order to check if its results support our decision to describe Trigonoschema gen. nov. as a new genus distinct from Mioscarta. This study includes 16 exemplars of Cercopidae as ingroup representing genera Eoscarta Breddin, 1902 (3), Mioscarta (3), Trigonoschema (3), Poeciloterpa (5), Jacobsoniella Melichar, 1914(1) and Wawi Soulier-Perkins \& Le Cesne, 2016 (1) and 14 terminals as outgroup selected from Aphrophoridae, Clastopteridae and Machaerotidae. Genera included in the ingroup are supposed to be phylogenetically as close as possible to Mioscarta and Trigonoschema gen. nov. in order to test our hypothesis. We have selected genera that belong to the same tribe, Rhinaulacini: Poeciloterpa, Eoscarta from the Philippines and Wawi from Papua New Guinea. Jacobsoniella is found in the Philippines but in the actual classification it is incertae sedis concerning its tribe placement. However, the male terminalia for this genus present the same characteristic sterno-lateral plates observed in most Rhinaulacini genera. For this reason, we used it in our analysis, included in the ingroup.

Most specimens sampled were stored into $95-100 \%$ ethanol otherwise dry. DNA extractions were conducted using standard protocols for QIAmp DNA microkit (Qiagen) from disarticulated legs. Intact voucher specimens from which the disarticulated legs were extracted were mounted on pins and are deposited in the MNHN. PCR reactions, with negative controls included to detect contamination, were conducted in $25 \mu 1$ volume using Taq DNA Polymerase, from Taq Core kit (Qiagen) under standard thermocycler protocols (for Histone 3, 18S and 28S: initial denaturation for 3 minutes at $94^{\circ} \mathrm{C}$, then 35 cycles of $94^{\circ} \mathrm{C}$ for 30 seconds, $49-56^{\circ} \mathrm{C}$ for 40 seconds and $72^{\circ} \mathrm{C}$ for 1 minute, then a final extension step at $72^{\circ} \mathrm{C}$ for 10 minutes, and finally held at $10^{\circ} \mathrm{C}$ before being removed from the cycler. For $\mathrm{CO} 1$, the thermal cycling protocol are the following: initial denaturation for 3 minutes at $94^{\circ} \mathrm{C}$; five cycles of 30 seconds at $94^{\circ} \mathrm{C}, 40$ seconds at $47^{\circ} \mathrm{C}$, and 1 minute at $72^{\circ} \mathrm{C} ; 30$ cycles of 30 seconds at $94^{\circ} \mathrm{C}$, 40 seconds at $52^{\circ} \mathrm{C}$, and 1 minute at $72^{\circ} \mathrm{C} ; 10$ minutes at $72^{\circ} \mathrm{C}$; and finally held at $10^{\circ} \mathrm{C}$ ) and using Oligonucleotide primers (Table 3) targeting four loci: CO1, Histone 3, 18S, and 28S.

Amplified DNA was visualized using 1-2\% agarose gel electrophoresis with midori green staining. Sequence fragments were imported into codoncode aligner V.5.1.4. (CodonCode Corporation, Dedham, Massachusetts, USA) and trimmed to remove primer sequence. After sequence inspection, contiguous sequences were assembled and edited based on chromatograms to ensure the accuracy of base calls. In addition, insertions/deletions and checking of contaminations were confirmed for accuracy by using a reference sequence for comparison in BLAST (https://blast.ncbi.nlm.nih.gov/Blast.cgi). All sequence data are accessioned into GenBank (Table 4).

\section{Sequence alignment and Phylogenetic reconstruction}

Consensus sequences were imported to Phylosuite v1.2.2 (Zhang et al. 2020) and aligned using MAFFT (Katoh \& Standley 2013) and MACSE (Ranwez et al. 2011, 2018). Nuclear protein coding genes (H3) were aligned with MAFFT program, using the FFT-NS-i algorithm. Ribosomal genes (18S and 28S) were aligned using MAFFT with algorithm E-INS-I (suitable for sequences with long unalignable regions). Mitochondrial protein coding genes (CO1) were aligned using MACSE.

For the combined analyses all sequence alignments were concatenated into a single data set using Phylosuite v1.2.2 (Zhang et al. 2020). The resulting data matrix consisted of $3575 \mathrm{bp}$ of DNA nucleotide sequence data for 30 Cercopiodea specimens used as terminals. Phylogenetic reconstructions were conducted using maximum likelihood (ML) criteria (Guindon et al. 2010) and Bayesian inference (BI). Under all reconstructions method, gaps were treated as missing data. Partitioned analyses were conducted with Partition Finder (Lanfear et al. 2012, 2016). Best fitting model was searched using partition finder with following configuration: branchlengths $=$ linked, models $=$ all, model_selection 
$\mathrm{AICc}$ and the search = greedy. Codon code mode was activated for $\mathrm{CO} 1$ before running the analysis. Results obtained with the corrected Akaike Information Criterion (AICc) (Guindon et al. 2010; Lanfear et al. 2012, 2016) indicated that GTR $+\mathrm{G}$ was best fitting model for Histone 3; TRNEF+I+G for 18S; $\mathrm{GTR}+\mathrm{I}+\mathrm{G}, \mathrm{TRN}+\mathrm{I}$ and $\mathrm{HKY}+\mathrm{I}+\mathrm{G}$ for each codon base for $\mathrm{CO} 1$ and $\mathrm{GTR}+\mathrm{I}+\mathrm{G}$ for $28 \mathrm{~S}$.

For ML analysis the results were then imported to IQ tree (Guindon et al. 2010; Minh et al. 2013; Nguyen et al. 2015; Hoang et al. 2018) for fast and effective stochastic algorithm to reconstruct phylogenetic trees. Partition mode was selected,"Models" argument will be ignored and models and thread were automatically set to auto. Maximum likelihood phylogeny were inferred under edge-linked partition model for 1000 standard bootstraps, as well as the Shimodaira-Hasegawa-like approximate likelihoodratio test (SH_aLRT branch test) with 1000 replicates (Guindon et al. 2010; Minh et al. 2013; Nguyen et al. 2015; Hoang et al. 2018).

BI analysis was conducted with MrBayes version 3.1.2 (Huelsenbeck \& Ronquist 2001; Ronquist \& Huelsenbeck 2003) as implemented in CIPRES (Miller et al. 2010). Because MrBayes only allows a relatively small collection of models, models in the analyses were approximated using $\mathrm{GTR}+\mathrm{I}+\mathrm{G}$ $(\mathrm{nst}=6)$ for DNA, or 'wag' for Protein (Guindon et al. 2010; Lanfear et al. 2012, 2016). The analysis coupled with Metropolis Coupled Markov Chain Monte Carlo (MCMCMC) was implemented. These analyses were conducted with six-gene partition implemented across two independent runs each with four chains run (one cold and three heated) for 20 million generations with 1000 sampling frequency. Burn in fraction value was set to 0.25 in which the initial $25 \%$ of sampled data were discarded as burn-in. The final posterior probability tree was calculated as a 50\% majority-rule consensus tree (Huelsenbeck \& Imennov 2002; Huelsenbeck et al. 2002). Clade support was evaluated by their posterior probabilities (BPP). The IQ Tree analysis tree and the Bayesian inference analysis tree were visualized with FIGTREE v1.1.3 (Rambaut 2016).

\begin{tabular}{lll}
\multicolumn{2}{l}{ Abbreviations used in text } \\
ae $=$ & apical extension \\
$\mathrm{Cua}=$ & cubital anterior vein \\
$\mathrm{Cup}$ & $=$ & cubital posterior vein \\
$\mathrm{IP}$ & $=$ & intermediate plate \\
$\mathrm{m}-\mathrm{cu}$ & $=$ & cross vein between Median and Cubital veins \\
$\mathrm{PCR}$ & $=$ & polymerase Chain Reaction \\
$\mathrm{pdp}$ & $=$ & postero-dorsal protrusion \\
$\mathrm{pp}$ & $=$ & posterior protrusion \\
$\mathrm{R}$ & $=$ & radial vein \\
$\mathrm{Ra}$ & $=$ & radial anterior vein \\
$\mathrm{Rp}$ & $=$ & Radial posterior vein \\
$\mathrm{SLP}$ & $=$ & sterno-lateral plate \\
$\mathrm{Sc}$ & $=$ & sub-costal vein
\end{tabular}

\section{Acronyms}

$\begin{array}{lll}\text { ECpc } & = & \text { Elorde Crispolon personal collection } \\ \text { MIZ } & = & \text { Museum and Institute of Zoology, Warszawa, Poland } \\ \text { MNHN } & = & \text { Muséum national d'Histoire naturelle, Paris, France } \\ \text { NHM } & = & \text { Natural History Museum, London, UK } \\ \text { SMTD } & = & \text { Staatliches Museum für Tierkunde, Dresden, Germany } \\ \text { UPLBMNH } & = & \begin{array}{l}\text { University of the Philippines Los Baños Museum of Natural History, } \\ \text { Philippines }\end{array}\end{array}$




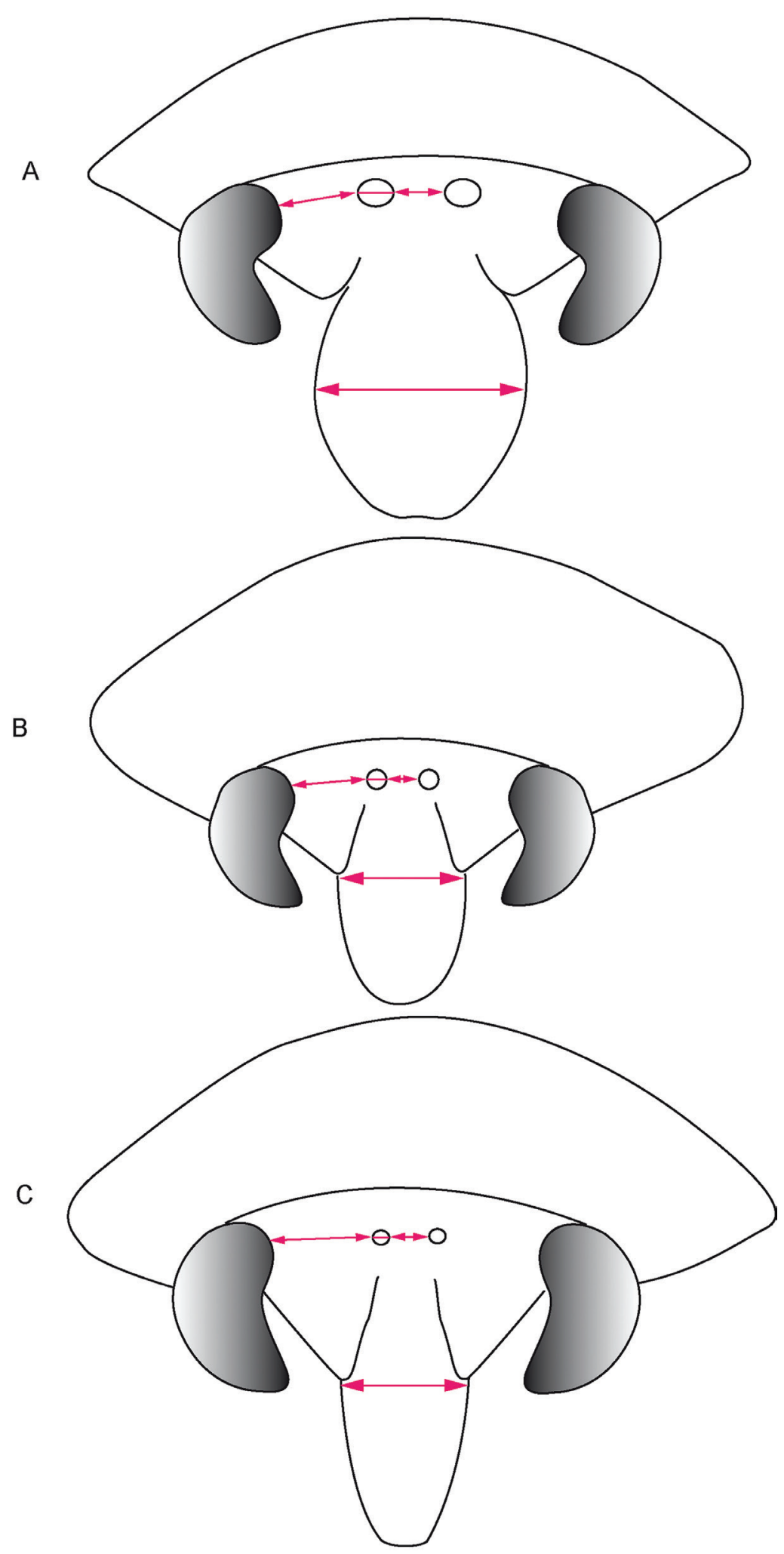

Fig. 1. Head schematic representation with diameter of ocelli compared to distance between them and between ocellus and compound eye. A. Mioscarta Breddin, 1901. B. Trigonoschema Crispolon \& Soulier-Perkins gen. nov. C. Poeciloterpa Stål, 1870. 

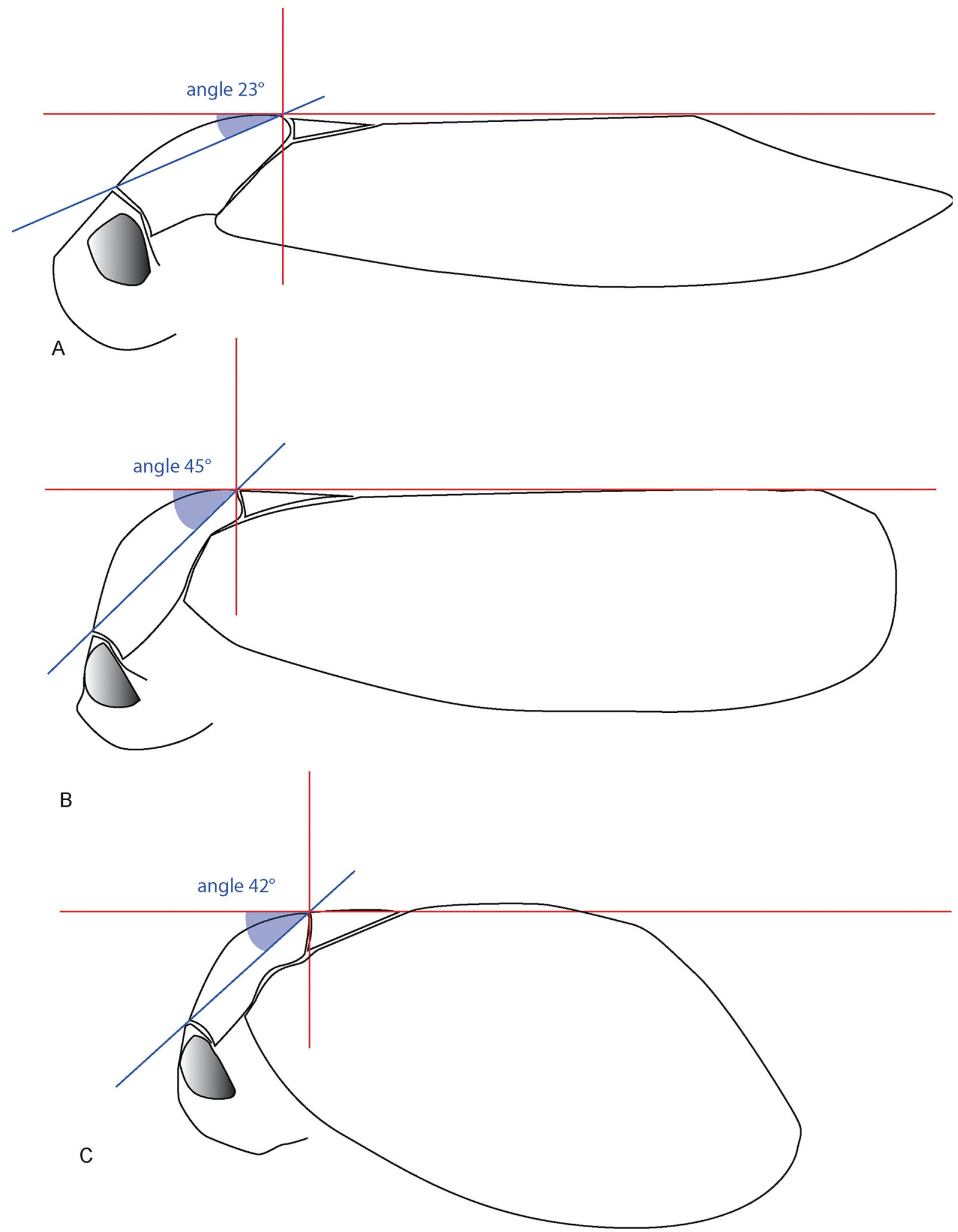

Fig. 2. Schematic representation of the pronotum angle. A. Mioscarta Breddin, 1901. B. Trigonoschema Crispolon \& Soulier-Perkins gen. nov. C. Poeciloterpa Stål, 1870. 


\title{
Results
}

\section{Taxonomy}

\author{
Class Insecta Linnaeus, 1758 \\ Order Hemiptera Linnaeus, 1758 \\ Suborder Auchenorrhyncha Duméril, 1806 \\ Infraorder Cicadomorpha Evans, 1946 \\ Family Cercopidae Leach, 1815 \\ Subfamily Cercopinae Oshanin, 1916 \\ Tribe Rhinaulacini Kirkaldy 1906 \\ Subtribe Poeciloterpina Schmidt, 1920
}

Genus Mioscarta Breddin, 1901

Mioscarta Breddin, 1901: 123 (new genus), 183 (Zoogeography).

\section{Type species}

Mioscarta forcipata Breddin, 1901.

\section{Diagnosis}

The genus can be identified by the following combination of characters:

Habitus general shape dorso-ventrally flattened, in lateral view total length nearly 4 times height (Figs 2, 4-11A). Pronotum angle not more than $25^{\circ}$ (Figs 2, 4-11A). Distance between ocellus and compound eye 2 times ocellus diameter (Fig. 1A). Ocelli large, distance between eyes less than 8 times ocellus diameter (Fig. 1A). Apical reticulation of the tegmen generally developed and reduced in few cases (Figs 4-11A-C). Widest part of postclypeus in frontal view is at midheight (Fig. 1A). Apical curve of tegmen visible in dorsal view (Figs 4-11C). Widest part of habitus in dorsal view at midlength of tegmen (Figs 4-11C). Male subgenital plates is at least 1.5 times longer than pygofer height. Male subgenital plates appendage always present, longer than main plate (Figs 5, 7-8, 10-11F).

Among the Rhinaulacini, Mioscarta closely resembles Trigonoschema Crispolon \& Soulier-Perkins gen. nov. with respect to the distance between ocelli, postclypeus shape in frontal view, postclypeus
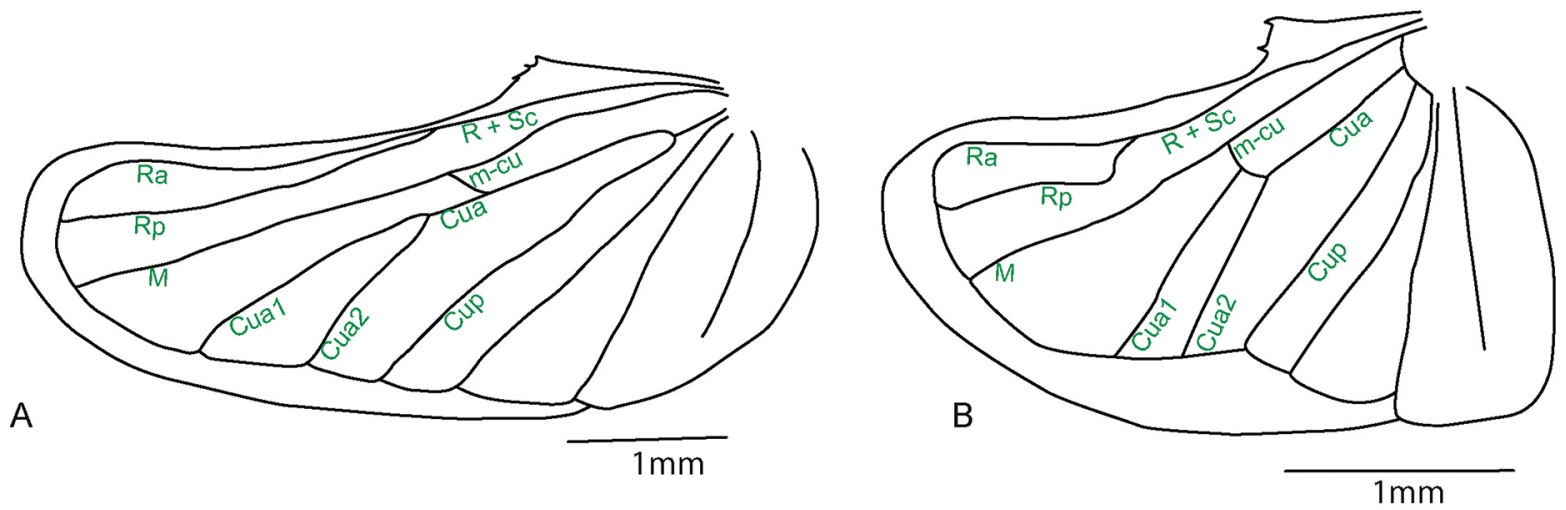

Fig. 3. Posterior wings. A. Mioscarta sp. B. Poeciloterpa sp. 
longitudinal groove, apical cells of the tegmen, Rp posterior wing, absence of r-m crossvein, and presence of sterno-lateral plate between subgenital plate and pygofer while Peociloterpa with respect to postclypeus longitudinal groove, absence of r-m crossvein, presence of sterno-lateral plate between subgenital plate and pygofer and paramere general shape, but they differ by the following characters presented in Table 2 below.

\section{Distribution}

India, Indonesia (Borneo, Flores and Sulawesi), Malaysia (Borneo), and Philippines.

\section{Key to the species of Philippine Mioscarta Breddin, 1901}

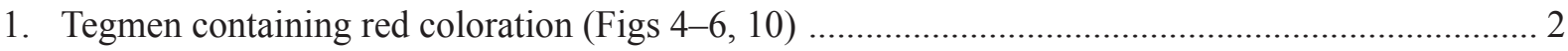

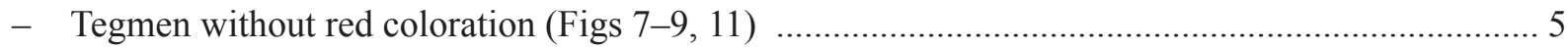

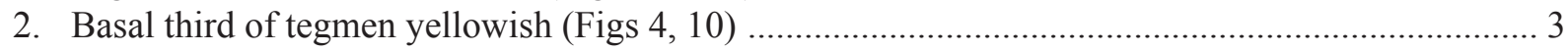

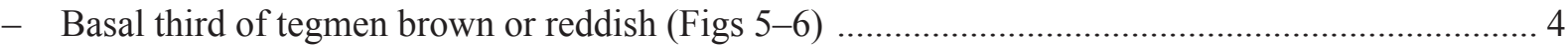

3. Narrow brown transverse band following basal third then tegmen getting darker and redder toward apex (Fig. 10)

M. semperi Jacobi, 1905

- No narrow transverse band but a brown patch within basal area, a large reddish band underlines costal and apical margins (Fig. 4)

M. basilana Jacobi, 1927

4. Basal third of tegmen reddish, rest of the tegmen darker brown with red underlining veins (Fig. 5)

- Tegmen with basal third brown getting lighter in second third and slightly darker in last apical third with red underlying veins (Fig. 6)

M. lutea Schmidt, 1925

5. Tegmen coloration containing some black (Figs 8-9)

- Tegmen coloration from yellowish to brown only (Figs 7, 11) ................................................. 7

6. Tegmen entirely black except for the very orange base (Figs 8-9) M. obscuripennis Schmidt, 1920

- Tegmen basally yellowish brown and apically black M. flavobasalis Jacobi, 1927

7. Tegmen brownish and opaque, darker toward apex, pronotum brownish (Fig. 7)

M. nubisa Crispolon \& Soulier-Perkins sp. nov.

- Tegmen yellow with some brown and translucent parts, pronotum yellowish with darker irregular patch in middle (Fig. 11) M. translucida Crispolon \& Yap sp. nov.

Mioscarta basilana Jacobi, 1927

Fig. 4

Mioscarta basilana Jacobi, 1927: 550.

Mioscarta basilana - Lallemand 1949: 84-85.

\section{Material examined}

PHILIPPINES • 3 우; “Island of Basilan Baker”; "1926”; “Typus”; “coll. A. Jacobi”, "basilana Jac.”, "Museum für Tierkunde Dresden (MTD)"; SMTD.

\section{Distribution}

Philippines: Mindanao: Basilan.

\section{Remarks}

The description provided by Jacobi (1927) was based on four females. Here we provide photographs of a female syntype and its labels. Male remains unknown. 


\section{Mioscarta ferruginea (Walker, 1851)}

Fig. 5

Triecphora ferruginea Walker, 1851: 672.

Cercopis ferruginea - Stål 1870: 721.

Eoscarta ferruginea - Distant 1908: 132.

Mioscarta ferruginea - Lallemand 1912: 299.

\section{Material examined}

PHILIPPINES • O; "Leyte Mt. Pangasugan Elev. 300 m; 6 May 1952; C. R. Baltazar", "Eoscarta sp. Bal 53" "UPLBMNH HEM-04043" • ô; "Mt. Maquiling Elev. 200 m.; 23 Jan. 1954; J.N. Gibe", "UPLBMNH HEM-04044” • đo+ ; “Odell Pltn. Tagum, Davao; 16 Jan. 1956; G.B. Viado", "UPLBMNH HEM-04045, 04047" • o; "Odell Pltn. Tagum, Davao; 17 Jan. 1956; S.M. Cendaña”; "UPLBMNH HEM-04046" • +; "Puting Lupa, Cal.; 3 Aug. 1986; Chantana B. coll.”; "UPLBMNH HEM-04997" • "Ex. grasses CES, Laguna; 2 Jan. 1981; M.A. Tabasa", "Cercopidae", "UPLBMNH HEM-04062" • O; "PH. MT. APO, Lake Agco, Kidapawan, Cotabato, Min; 30 Jul. 2015; E.S. Crispolon", "Muséum Paris; MNHN (EH) 24853" • ${ }^{\top}$; ; "PH. Lake Sebu, South Cotabato, Mindanao; 20 Jul. 2016; E.S. Crispolon"; "Muséum Paris; MNHN (EH) 24854 to 24855" - 2 q ; "PH. Paniki Falls, Balabag, Kidapawan,

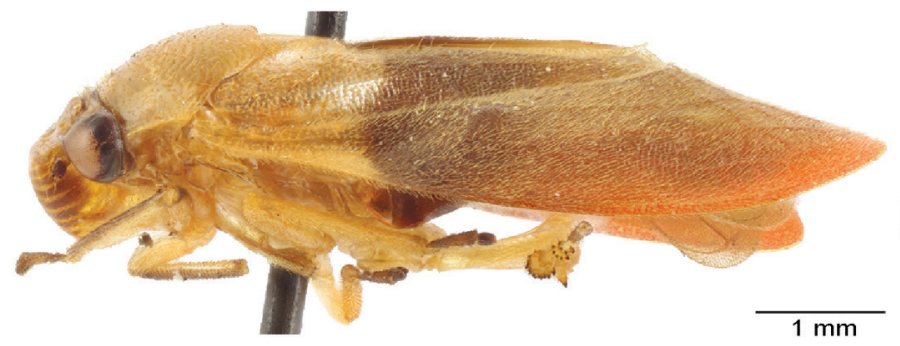

A

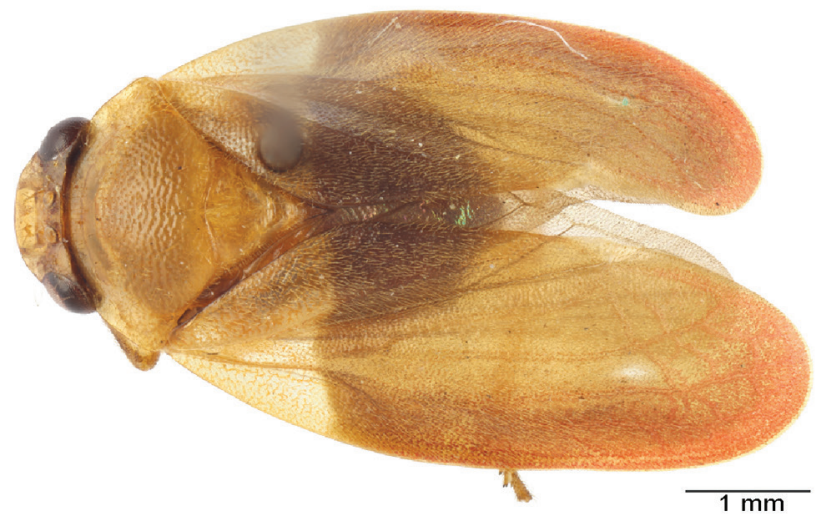

C

Fig. 4. Mioscarta basilana Jacobi, 1927 q syntype habitus. A. Lateral view. B. Frontal view. C. Dorsal view. D. Labels.

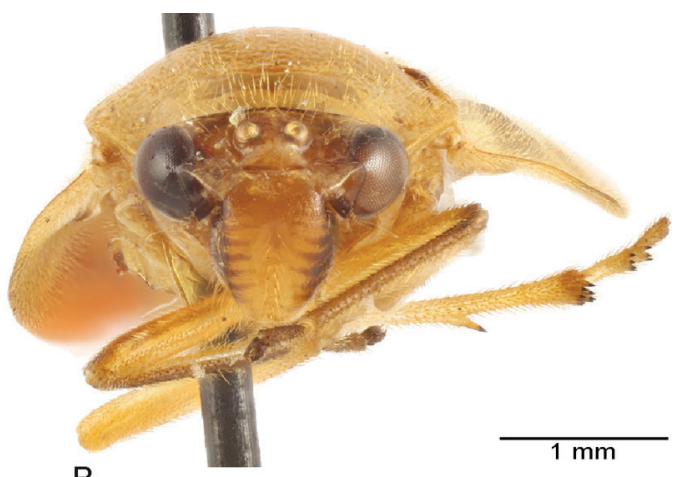

B

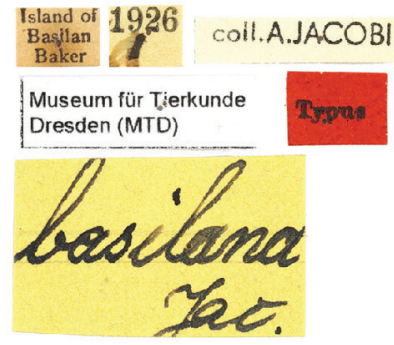

D 

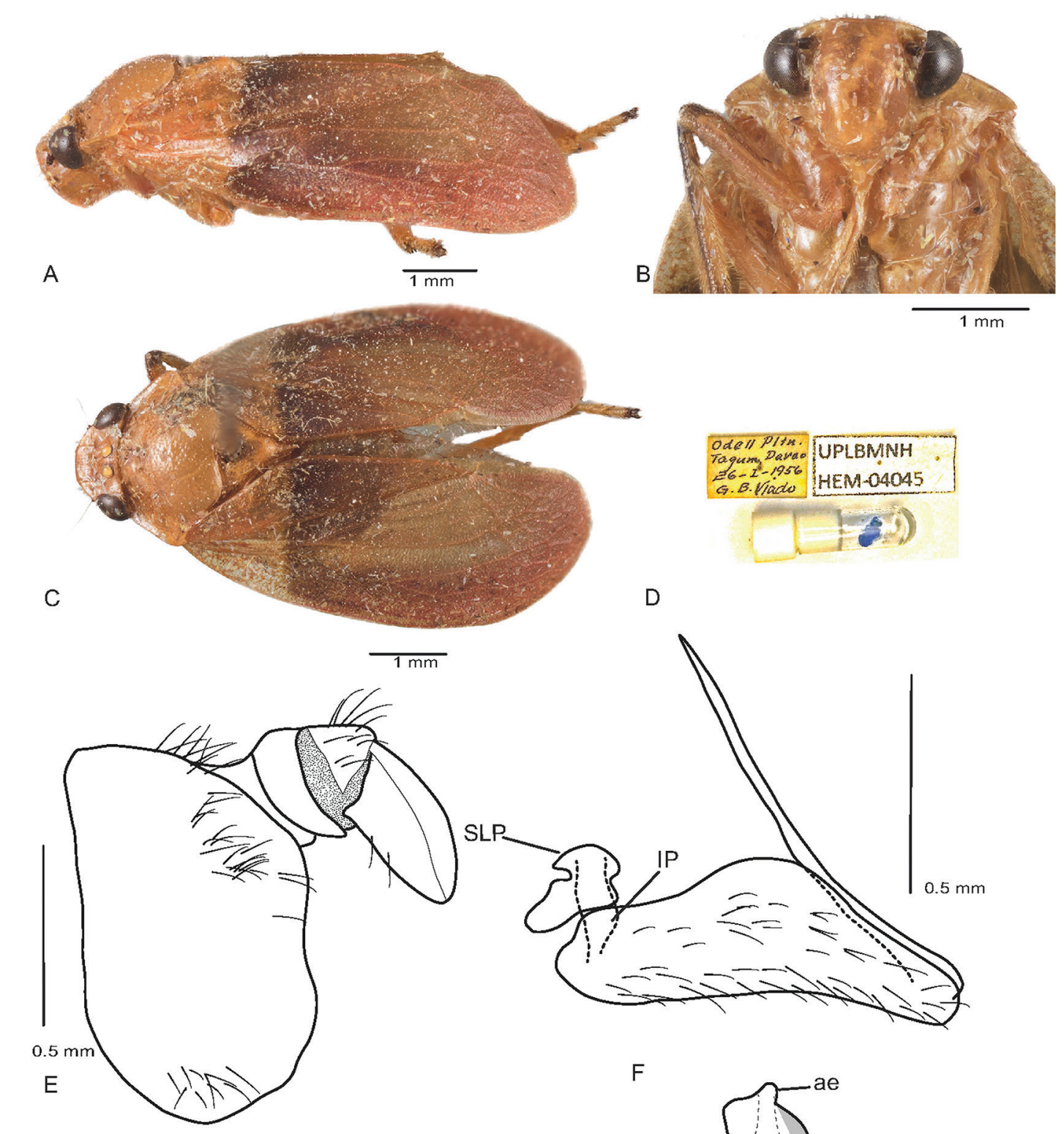

$\mathrm{D}$

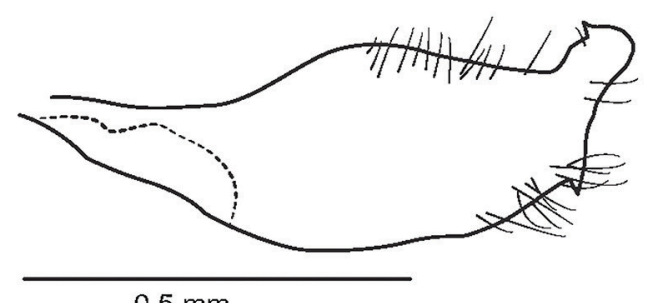

G

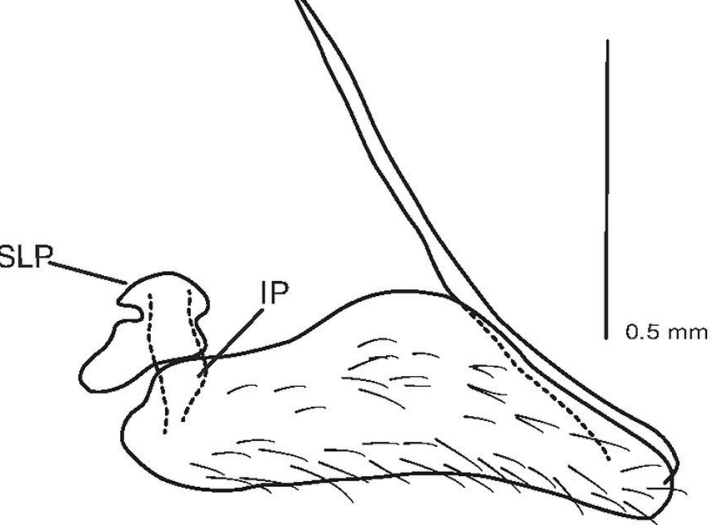

$\mathrm{F}$

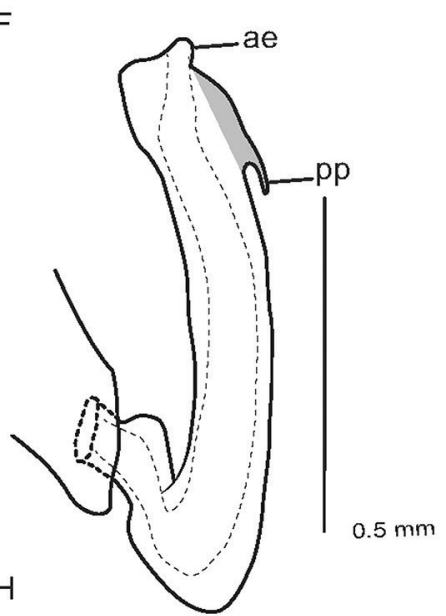

Fig. 5. Mioscarta ferruginea (Walker, 1851) habitus and $\widehat{\partial}$ terminalia in lateral view. A. Lateral view. B. Frontal view. C. Dorsal view. D. Labels. E. Pygofer and anal tube. F. Sterno-lateral, intermediate and subgenital plates. G. Paramere. H. Aedeagus. 
Table 2. Character diagnosis for the three closely related genera from the Philippines.

\begin{tabular}{|c|c|c|c|c|}
\hline \multirow{2}{*}{\multicolumn{2}{|c|}{ No. Characters }} & \multicolumn{3}{|c|}{ Genera } \\
\hline & & Mioscarta & Poeciloterpa & Trigonoschema gen. nov. \\
\hline 1 & $\begin{array}{l}\text { Habitus general shape in } \\
\text { lateral view }\end{array}$ & $\begin{array}{l}\text { dorso-ventrally flattened, } \\
\text { total length nearly } 4 \text { times } \\
\text { height (Figs 2, 4-11A) }\end{array}$ & $\begin{array}{l}\text { globulous, total length } \\
\text { around } 2.5 \text { times height } \\
\text { (Fig. 2C) }\end{array}$ & $\begin{array}{l}\text { not dorso-ventrally } \\
\text { flattened, in lateral view } \\
\text { total length around } 3 \\
\text { times height } \\
\text { (Figs 2, 12-16B) }\end{array}$ \\
\hline 2 & $\begin{array}{l}\text { Total lenght of the } \\
\text { specimen }\end{array}$ & $8 \mathrm{~mm}-11 \mathrm{~mm}$ & not exceeding $8 \mathrm{~mm}$ & $9.5 \mathrm{~mm}-12.5 \mathrm{~mm}$ \\
\hline 3 & Pronotum angle & $\begin{array}{l}\text { not more than } 25^{\circ} \\
\text { (Figs } 2,4-11 \mathrm{~A} \text { ). }\end{array}$ & $42^{\circ}$ (Fig. 2C) & $\begin{array}{l}\text { around } 45^{\circ} \\
\text { (Figs } 2 \mathrm{~B}, 12-16 \mathrm{~A})\end{array}$ \\
\hline 4 & $\begin{array}{l}\text { Distance between ocellus } \\
\text { and compound eye }\end{array}$ & $\begin{array}{l}2 \text { times ocellus diameter } \\
\text { (Fig. 1A) }\end{array}$ & $\begin{array}{l}6 \text { times ocellus diameter } \\
\text { (Fig. 1C) }\end{array}$ & $\begin{array}{l}\text { less than } 4 \text { times ocellus } \\
\text { diameter (Fig. 1B). }\end{array}$ \\
\hline 5 & Distance between ocelli & $\begin{array}{l}1.5 \text { ocellus diameter } \\
\text { (Fig. 1A) }\end{array}$ & $\begin{array}{l}\text { at least } 2 \text { times ocellus } \\
\text { diameter (Fig. 1C) }\end{array}$ & $\begin{array}{l}1.5 \text { ocellus diameter } \\
\text { (Fig. 1B) }\end{array}$ \\
\hline 6 & $\begin{array}{l}\text { Size of ocelli evaluated } \\
\text { according to the distance } \\
\text { between eyes using ocellus } \\
\text { diameter }\end{array}$ & $\begin{array}{l}\text { large, distance between eyes } \\
\text { less than } 8 \text { times ocellus } \\
\text { diameter (Fig. 1A) }\end{array}$ & $\begin{array}{l}\text { very small, distance } \\
\text { between eyes } 16 \\
\text { times ocellus diameter } \\
\text { (Fig. 1C) }\end{array}$ & $\begin{array}{l}\text { small, distance between } \\
\text { eyes 9-10.5 times ocellus } \\
\text { diameter (Fig. 1B) }\end{array}$ \\
\hline 7 & $\begin{array}{l}\text { Postclypeus shape } \\
\text { in frontal view }\end{array}$ & ovoid (Fig. 1A) & $\begin{array}{l}\text { compressed laterally } \\
\text { with lateral sides almost } \\
\text { straight (Fig. 1C) }\end{array}$ & ovoid (Fig. 1B) \\
\hline 8 & $\begin{array}{l}\text { Widest part of postclypeus } \\
\text { in frontal view }\end{array}$ & mid height (Fig. 1A) & close to frons (Fig. 1C) & close to frons (Fig. 1B) \\
\hline 9 & $\begin{array}{l}\text { Apical reticulation } \\
\text { of the tegmen }\end{array}$ & $\begin{array}{l}\text { generally developed and } \\
\text { reduced in few cases } \\
\text { (Figs } 4-11 \mathrm{~A}, \mathrm{C} \text { ) }\end{array}$ & reduced & reduced (Figs 12-16A, C) \\
\hline 10 & $\begin{array}{l}\text { Apical curve of tegmen in } \\
\text { dorsal view }\end{array}$ & visible (Figs 4-11C) & rarely visible & $\begin{array}{l}\text { rarely visible } \\
\text { (Figs 12-16C) }\end{array}$ \\
\hline 11 & Widest part of habitus & $\begin{array}{l}\text { midlength of tegmen } \\
\text { (Figs 4-11C) }\end{array}$ & $\begin{array}{l}\text { before midlength of } \\
\text { tegmen }\end{array}$ & $\begin{array}{l}\text { before midlength of } \\
\text { tegmen (Figs 12-16C) }\end{array}$ \\
\hline 12 & Apical cells of the tegmen & not concave (Figs 4-11A, C) & concave & $\begin{array}{l}\text { not concave } \\
\text { (Figs } 12-16 \mathrm{~A}, \mathrm{C})\end{array}$ \\
\hline 13 & Rp posterior wing & $\begin{array}{l}\text { Rp separating from } \mathrm{SC}+\mathrm{Ra} \\
\text { nearly at midlength } \\
\text { (Fig. 3A) }\end{array}$ & $\begin{array}{l}\text { Rp separating from } \\
\text { SC+Ra after midlength } \\
\text { and making a strong } \\
\text { bend after separating } \\
\text { from SC+ Ra (Fig. 3B) }\end{array}$ & $\begin{array}{l}\text { Rp separating from } \\
\text { SC+Ra nearly at } \\
\text { midlength, } \\
\text { (Fig. 3A (Fig. 13E)) }\end{array}$ \\
\hline 14 & Subgenital plates length & $\begin{array}{l}\text { at least } 1.5 \text { times longer than } \\
\text { pygofer height } \\
\text { (Figs } 5,7-8,10-11 \mathrm{~F} \text { ). }\end{array}$ & $\begin{array}{l}\text { slightly longer than } \\
\text { pygofer height }\end{array}$ & $\begin{array}{l}\text { clearly shorter than } 1.5 \\
\text { times longer than pygofer } \\
\text { height (Figs } 12,14,16 \mathrm{~F} \text { ) }\end{array}$ \\
\hline 15 & $\begin{array}{l}\text { Subgenital plates fine } \\
\text { appendage }\end{array}$ & $\begin{array}{l}\text { always present, longer than } \\
\text { the main plate } \\
\text { (Figs } 5,7-8,10-11 \mathrm{~F} \text { ). }\end{array}$ & $\begin{array}{l}\text { generally absent, if } \\
\text { present shorter than the } \\
\text { main plate }\end{array}$ & $\begin{array}{l}\text { generally present but } \\
\text { shorter than the main } \\
\text { plate (Figs } 12,14 \& 16 \mathrm{~F} \text { ) }\end{array}$ \\
\hline 16 & Paramere general shape & $\begin{array}{l}\text { not globose and without } \\
\text { protrusion on lateral side } \\
\text { (Figs } 5,7-8,10-11 \mathrm{G} \text { ). }\end{array}$ & $\begin{array}{l}\text { not globose and without } \\
\text { protrusion on lateral side }\end{array}$ & $\begin{array}{l}\text { globose with protrusion } \\
\text { e on lateral side } \\
\text { (Figs } 12,14,16 \mathrm{G}) \text {. }\end{array}$ \\
\hline
\end{tabular}


CRISPOLON E. Jr. S. et al., New genus and new species of spittlebugs from the Philippines

Cotabato, Min; 18 March 2017; N. Melano", "Muséum Paris, MNHN (EH) 24856 to 24857” • 9 ; "PH.

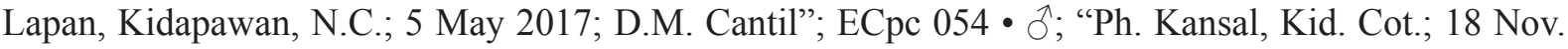
2017; Pasion C.A."; ECpc 056 • O'; "PH. Lake AGCO; 21 Dec. 2017; ESC"; Philippines; ECpc 057 • O'; "Philippines, Mt. Apo, Bongolanon, Magpet N. Cotabato"; "27 to 28 Jun. 2019; JS Ramos"; "séquençage par Elorde Crispolon C-00027"; "Muséum Paris, MNHN (EH) 24858".

\section{Description}

\section{Male Terminalia}

In lateral view, posterior margin of pygofer slightly undulating (Fig. 5E). Subgenital plate (Fig. 5F) very long relative to height of pygofer with fine tapering appendage slightly longer than main plate, dorsal margin of plate largely rounded, sterno-lateral plate present, largely concave subapically on dorsal and ventral margins. Intermediate plate present, rectangular, linking internal side of lateral and subgenital plates. Paramere (Fig. 5G) not globose, dorsal margin rounded and regularly curving, apex with a sharp spine pointing dorsally, ventral margin regularly rounded with subapical part bearing a spiniform process pointing ventrally. Aedeagus (Fig. $5 \mathrm{H}$ ) with basal third of dorsal margin angled and sharply bent in acute angle and last $2 / 3$ vertical and roughly C-shaped, slightly humped in apical part of dorsal margin before apical extension, apical extension pointing postero-dorsally, posterior protrusion thin and hook-shaped, postero-dorsal protrusion absent.

\section{Type Locality}

Philippines: Visayas: Samar.

\section{Distribution}

Philippines: Luzon Island, Visayas: Samar and Leyte, Mindanao Island.

\section{Remarks}

Walker (1851) based his description on two male specimens "a" and "b" from the "Philippine Islands" coming from the collections of Mr Cuming and Mr Wood. Both specimens are currently kept in the NHM. According to Walker' description and Lallemand' key (1949), we identified specimens, for which we provide photographs and male terminalia description and drawings.

Mioscarta lutea Schmidt, 1925

Fig. 6

Mioscarta lutea Schmidt, 1925: 36.

Mioscarta lutea - Lallemand 1949: 84-85.

\section{Type Locality}

Philippines: Luzon: Baguio, Benguet province.

\section{Distribution}

Philippines: Luzon Island.

\section{Remarks}

Schmidt (1925) described this species based on a single female specimen. Therefore, the description does not contain any information on the male terminalia. Here, we provide photographs of the female holotype and labels. Male remains unknown. 
Mioscarta nubisa Crispolon \& Soulier-Perkins sp. nov. urn:1sid:zoobank.org:act:17979C2C-D071-44EA-9D16-974A742808A1

Fig. 7

\section{Diagnosis}

General shape of $M$. nubisa is similar to M. obscuripennis but are distinctly different in color. M. nubisa in dorsal view presents a brownish and opaque tegmen, darker toward apex, pronotum brownish and yellowish or brown legs while M. obscuripennis has a dark brown or black tegmen, brown or orange pronotum and orange legs.

\section{Etymology}

Species name refers to the light to darker coloration forming a cloudy pattern on the tegmen and is the female superlative of the latin word "nubis" which means cloudy.

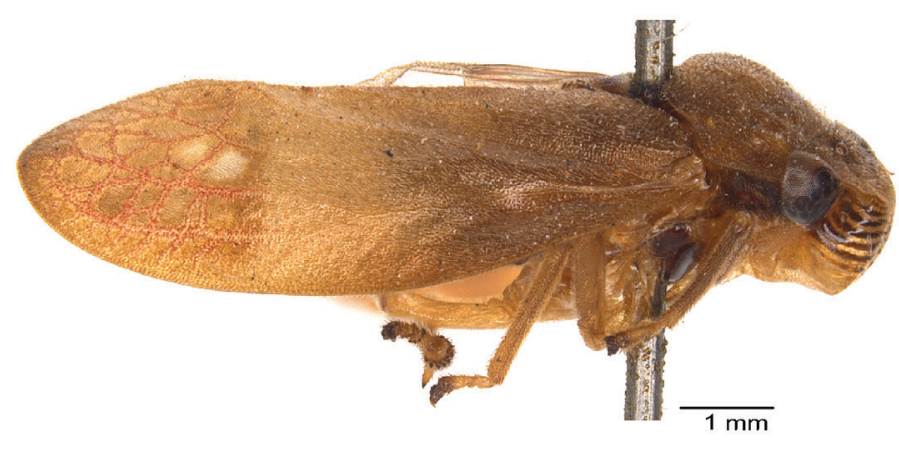

A

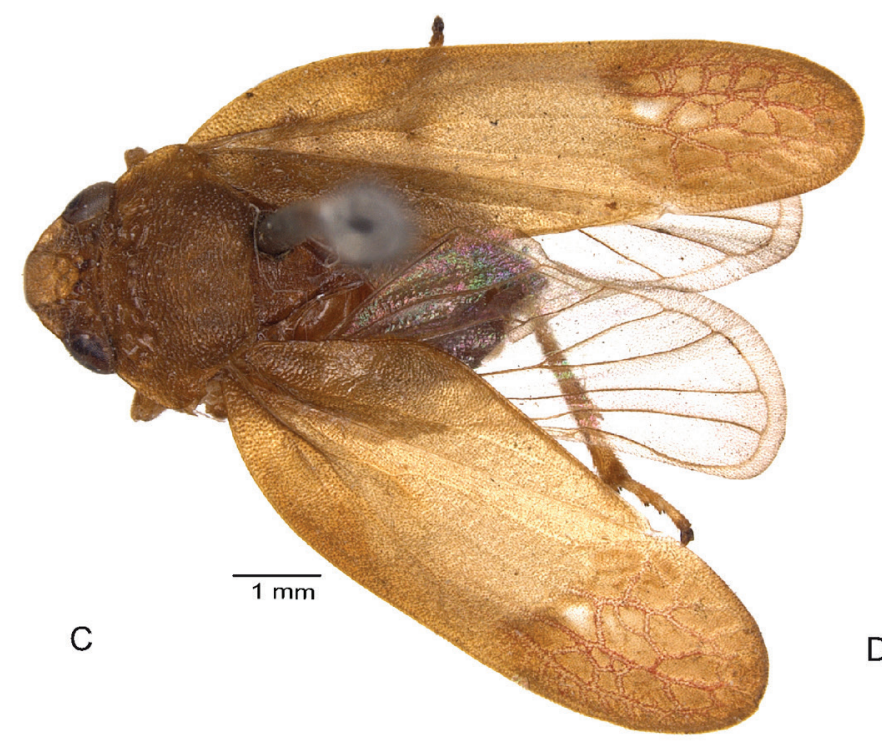

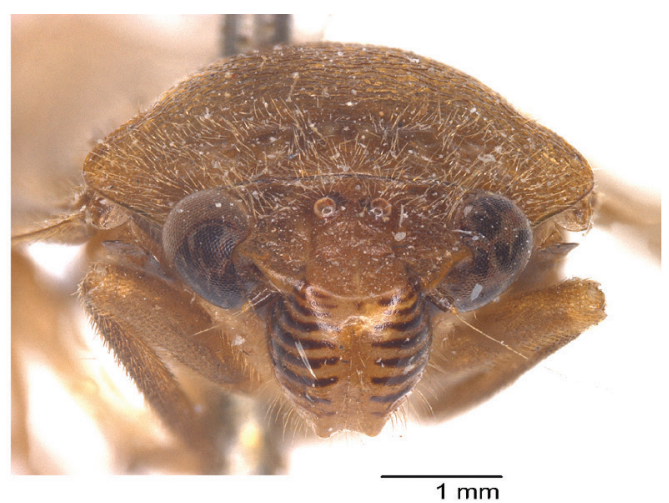

B

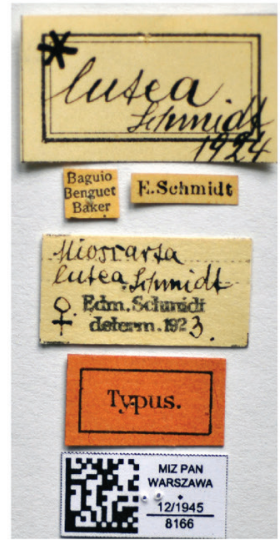

D

Fig. 6. Mioscarta lutea Schmidt, 1925, $q$ holotype habitus. A. Lateral view. B. Frontal view. C. Dorsal view. D. Labels. 
CRISPOLON E. Jr. S. et al., New genus and new species of spittlebugs from the Philippines

\section{Material examined}

\section{Holotype}

PHILIPPINES • \} \text { ; "Philippines, Camarines Sur, Luzon isl. Mt Isarog Natural Park, Panicuason Naga"; } "Muséum Paris; 1 May 2011; S. A. Yap, M.V. Yngente, O.L. Eusebio rec.”; UPLBMNH HEM-05052"; UPLBMNH.

\section{Paratypes}

PHILIPPINES • O; " "Trinidad; 22 June 1951; C.R. Baltazar"; "UPLBMNH HEM 04064”; UPLBMNH - O; $^{\text {; }}$ "Philippines, Camarines Sur, Luzon isl. Mt Isarog Natural Park, Panicuason Naga"; "Muséum Paris, 1 May 2011; S.A. Yap, M.V. Yngente, O.L. Eusebio rec."; UPLBMNH HEM-05053"; UPLBMNH - 16 ô.; "Philippines, Luzon, mount Isarog, Light trap"; "Muséum Paris; 31 May 2011; S. Yap Rec."; "Muséum Paris, MNHN (EH) 23645 to 23646, 23648, 24748, 24859 to 24870"; MNHN • O'; "Philippines, Luzon isl, Laguna, Los Banos, Mount Makiling; 14082' 29" N, 121²12.242" E; "Muséum Paris; 7 Nov. 2008; 605 m, Primary forest, at sight, A. Soulier-Perkins, S. Yap Rec."; "Muséum Paris, MNHN (EH) 23644"; MNHN.

\section{Description}

Body. Length $10 \mathrm{~mm}$ (tegmina included), width $5 \mathrm{~mm}$.

Head (Figs 1A, 7B). In dorsal view, large ocelli, distance between eyes less than 8 times ocellus diameter, distance between ocelli equals one ocellus diameter, distance between ocellus and compound eye 2 times ocellus diameter, ocelli closer to each other than from compound eyes. Eyes not prominent, length 1.44 times than wide. Vertex and frons longitudinal median carina absent. Vertex slightly longer than wide with 3 times ocellus diameter in between two vertex grooves outside ocelli and 3.5 times ocellus diameter between anterior and posterior vertex margins. Postclypeus with longitudinal furrow, slightly swollen and ovoid shape in frontal view, widest part at mid height (Figs 1A, 7B), not receding and prior to anteclypeus where it bends forming obtuse angle in lateral view (Fig. 7A). Rostrum long, reaching but not surpassing mesocoxae. Thorax (Figs 2A, 7A-C). In dorsal view, pronotum with anterior concavities on each side, anterior margin of pronotum as wide as posterior margin of head including eyes, anterolateral margin curved, posterior margin grooved, postero-lateral margin slightly concave, longer than anterolateral margin, humeral angle rounded. In lateral view, pronotum curving not more than $25^{\circ}$ (Figs 2, 7A). Scutellum as long as wide with large median dimple (Fig. 7C). Tegmen (Fig. 7A-C). R bifurcates on apical half, $\mathrm{M}$ bifurcate on basal third, apical reticulation well developed without concave apical cells. Posterior wing (Fig. 3A), Rp separating from SC+Ra nearly at midlength, $\mathrm{M}$ reaches ambient vein, Cua and Cup fused at base and $\mathrm{m}-\mathrm{cu}$ links $\mathrm{M}$ to Cua before Cua bifurcation, common base for Cup and Cua originate at base of wing, posterior wing with 7 longitudinal veins and 5 apical cells between $\mathrm{SC}+\mathrm{Ra}$ and $\mathrm{Cup}$, angular protrusion of costal margin near its base present. Metafemur with apical spine in inner margin, metatibiae bearing 1 lateral spine.

MaLE TERMinalia. In lateral view, posterior margin of pygofer largely convex in middle with slight curved on last third (Fig. 7E). Subgenital plates (Fig. 7F) very long relative to height of pygofer with fine tapering appendage longer than main plate, dorsal and ventral margin of main plate straight, sterno-lateral plate present, slightly elongated. Intermediate plate present, elongated, roughly oblong shaped, linking internal sides of lateral and subgenital plates. Paramere (Fig. 7G) not globose, dorsal margin convex and regularly curving finishing by a sharp process pointing dorsally, ventral margin convex and largely angled subapically, apex with spiniform process pointing postero-ventrally. Aedeagus (Fig. $7 \mathrm{H}$ ) with basal third of dorsal margin regularly bent without angle before the bent part, last $2 / 3$ vertical and S-shaped, ventral margin regularly curved, apical extension pointing posteriorly, posterior protrusion axe-shaped with edge prolonged ventrally by a straight, long and thin extension, postero-dorsal protrusion absent. 


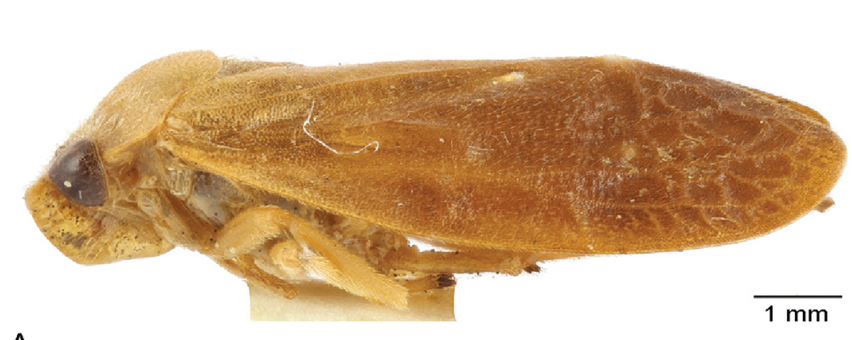

A

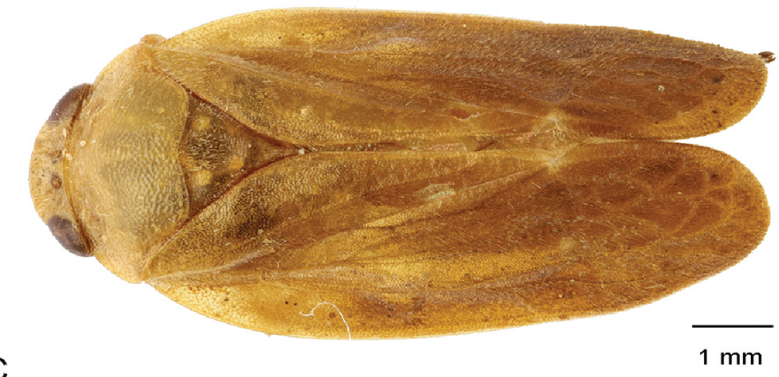

C
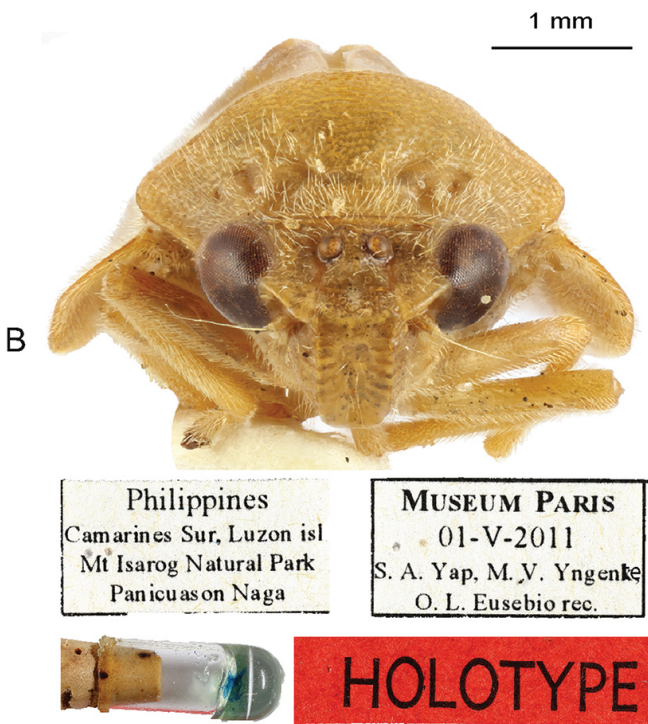

D
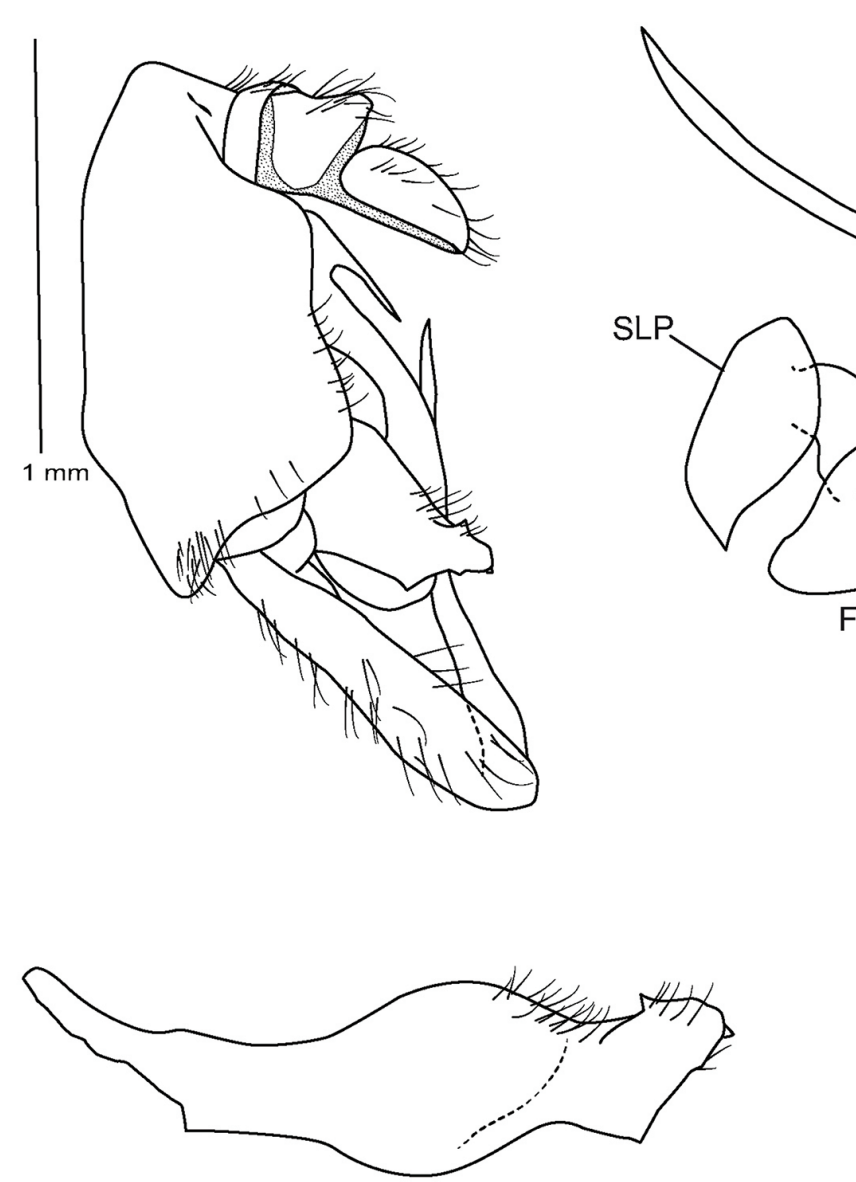

G

$0.5 \mathrm{~mm}$

$\mathrm{H}$

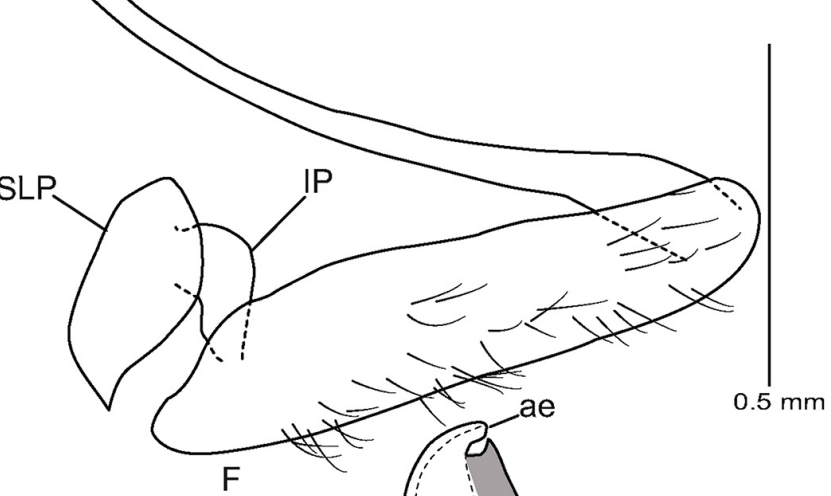

$\mathrm{F}$

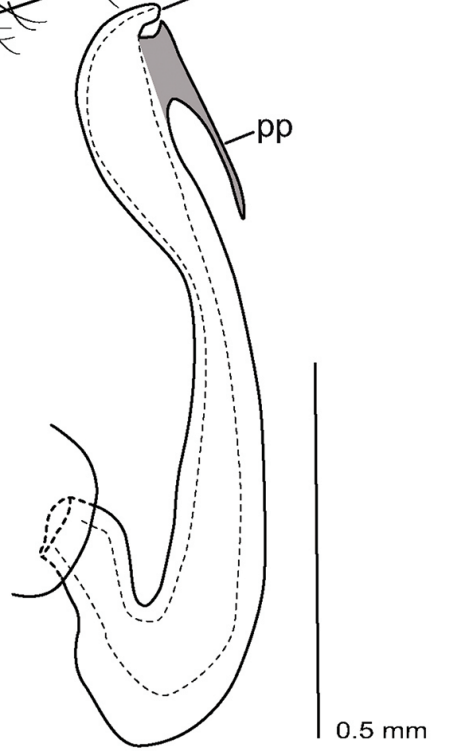

Fig. 7. Mioscarta nubisa Crispolon \& Soulier-Perkins sp. nov. holotype, habitus and $\partial$ terminalia in lateral view. A. Lateral view. B. Frontal view. C. Dorsal view. D. Labels. E. Terminalia. F. Sternolateral, intermediate and subgenital plates. G. Paramere. H. Aedeagus. 
CRISPOLON E. Jr. S. et al., New genus and new species of spittlebugs from the Philippines

Table 3. Primers used for amplifying and sequencing the molecular markers.

\begin{tabular}{|c|c|c|}
\hline Primers & Sequence & References \\
\hline \multicolumn{3}{|c|}{ Mitochondrial coding genes } \\
\hline \multicolumn{3}{|l|}{ COI } \\
\hline LCO1490-JJ & CHACWAAYCATAAAGATATYGG & Astrin \& Stûben 2008 \\
\hline HCO2198-JJ & AWACTTCVGGRTGVCCAAARAATCA & Astrin \& Stûben 2008 \\
\hline \multicolumn{3}{|c|}{ Nuclear Ribosomal genes } \\
\hline \multicolumn{3}{|l|}{$18 \mathrm{~S}$} \\
\hline a2.0 (F) & ATGGTTGCAAAGCTGAAAC & Whiting et al. 1997 \\
\hline 9R (R) & GATCCTTCCGCAGGTTCACCTAC & Whiting 2002 \\
\hline \multicolumn{3}{|l|}{$28 \mathrm{~S}$} \\
\hline $\mathrm{EE}(\mathrm{F})$ & CCGCTAAGGAGTGTGTAA & Hillis \& Dixon 1991; Cryan et al. 2000 \\
\hline $\mathrm{MM}(\mathrm{R})$ & GAAGTTACGGATCTARTTTG & Hillis \& Dixon 1991; Cryan et al. 2000 \\
\hline Lalt (F) & CCTCGGACCTTGAAAATCC & Dietrich et al. 2001 \\
\hline Galt (R) & TGTCTCCTTACAGTGCCAGA & Dietrich et al. 2001 \\
\hline $\mathrm{V}(\mathrm{F})$ & GTAGCCAAATGCCTCGTCA & \\
\hline $\mathrm{X}(\mathrm{R})$ & CACAATGATAGGAAGAGCC & Hillis \& Dixon 1991; Cryan et al. 2000 \\
\hline \multicolumn{3}{|c|}{ Nuclear protein coding genes } \\
\hline Histone 3 & & \\
\hline $\operatorname{HexAF}(\mathrm{F})$ & ATGGCTCGTACCAAGCAGACGGC & Colgan et al. (1998) \\
\hline HexAR (R) & ATATCCTTGGGCATGATGGTGAC & Colgan et al. (1998) \\
\hline
\end{tabular}

CoLOR. Head, antennae, pronotum, abdomen, legs scutellum light brown, rostrum brownish highlighted with white. Tegmen opaque light brown to brown.

\section{Type locality}

Philippines: Camarines Sur, Luzon isl. Mt Isarog Natural Park, Panicuason Naga.

\section{Distribution}

Philippines: Luzon Island.

Mioscarta obscuripennis Schmidt, 1920

Figs 8-9

Mioscarta obscuripennis Schmidt, 1920: 47.

Mioscarta obscuripennis - Lallemand 1949: 84-85.

\section{Material examined}

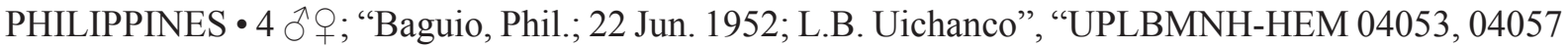

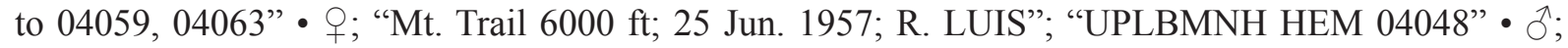
"EX. grasses; 27 Jan. 1980; NBG REAL, QUEZON J.B. BALATIBAT "UPLBMNH-HEM 04060" - Õ; "MSAC; 12 Aug. 1984; A.U. MANCIA", "UPLBMNH-HEM 04990" • 19 Aug. 1989; VP Gapud"; "UPLBMNH-HEM 04991" • ○; "MSAC; 12 Aug. 1984; A.U. MANCIA"; "UPLBMNH-HEM 04992" • 2 ふふふ; "Mt. Polis, Baubo Mtn. Province 22 Dec. 2000; Shem Padua"; "UPLBMNH-HEM 04993 to 04994" - ô; "QUEZON REAL Nat. Bot. Garden; 1-2 Apr. 1977; V.P.GAPUD"; "UPLBMNH-HEM 04995" • क; "Philippines, Negros volcan Canlaon, champ de coqs, $10^{\circ} 25^{\prime} 29^{\prime \prime}$ N, 12305'36" E"; "Muséum Paris, 1050 m; 28 Oct. 2010; D. Ouvrard Rec.", "Muséum 


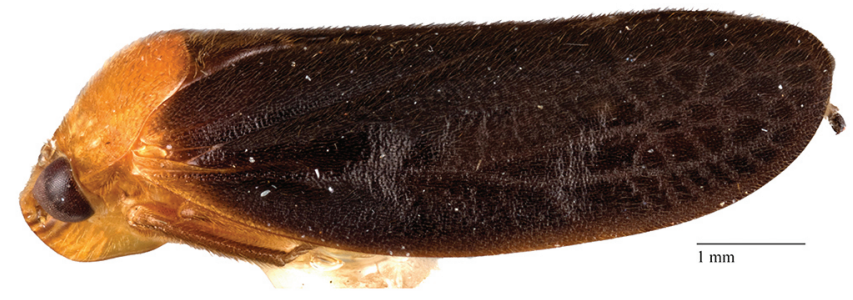

A

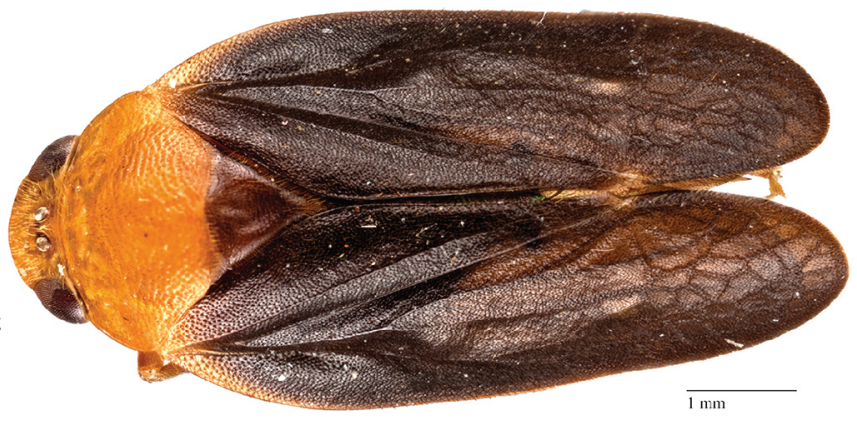

B
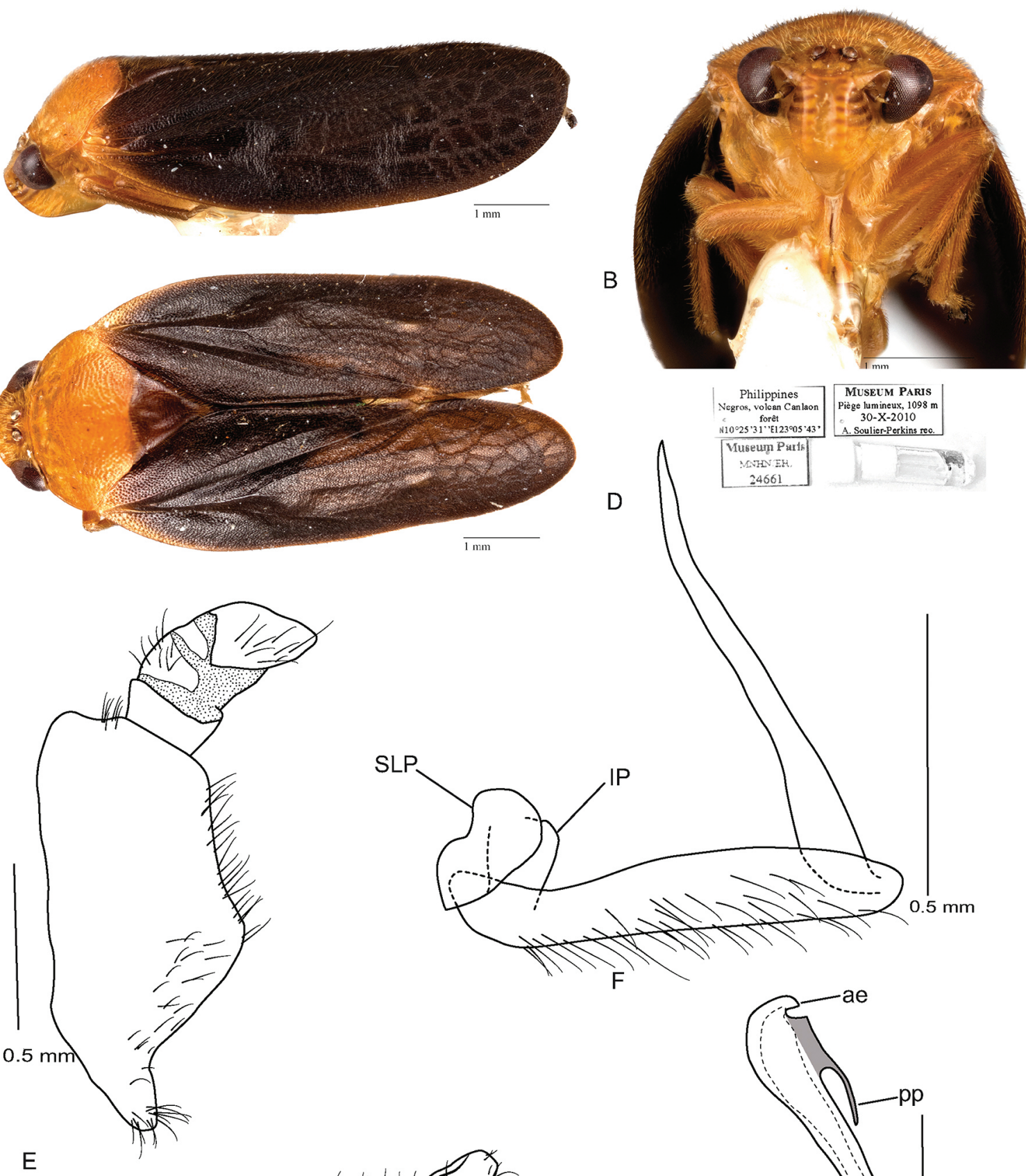

$\mathrm{D}$

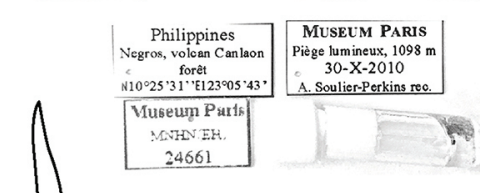

E

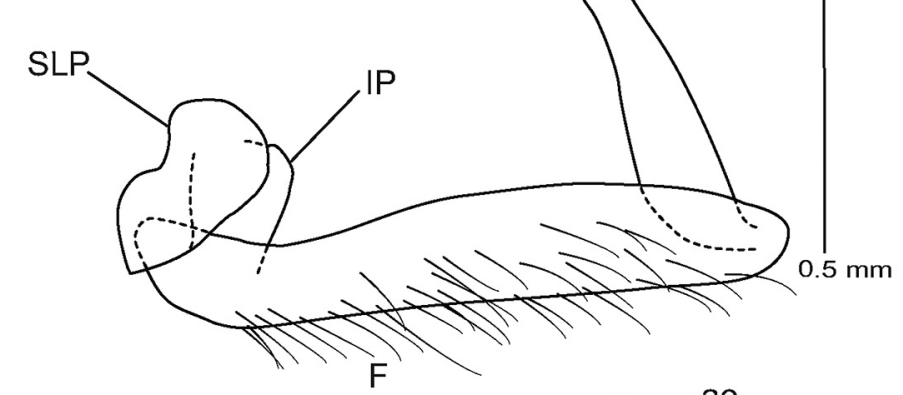

$\mathrm{F}$

$\mathrm{H}$

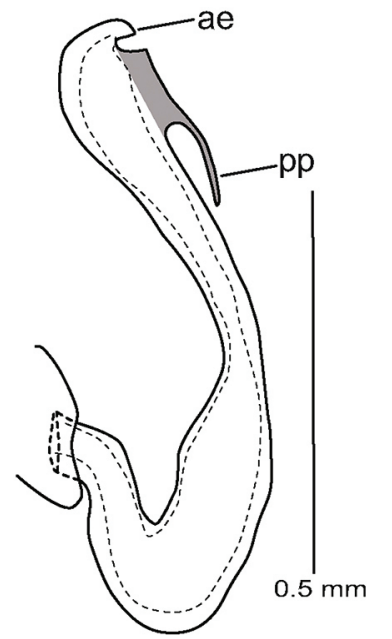

Fig. 8. Mioscarta obscuripennis Schmidt, 1920, habitus and $ð$ terminalia in lateral view. A. Lateral view. B. Frontal view. C. Dorsal view. D. Labels. E. Pygofer and anal tube. F. Sterno-lateral, intermediate and subgenital plates. G. Paramere. H. Aedeagus. 
Paris, MNHN (EH) 24809" • + ; "Philippines, Negros volcan Canlaon, champ de coqs, 10²5'29" N, 12305'36" E"; "Muséum Paris, 1050 m; 28 Oct. 2010; A Soulier-Perkins Rec."; "Muséum Paris, MNHN

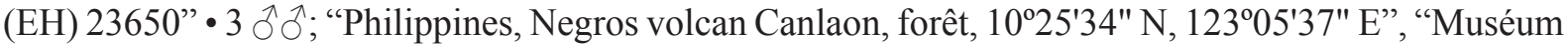

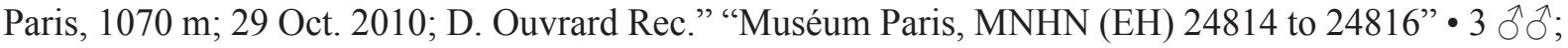
"Philippines, Negros volcan Canlaon, forêt; 10²5'31" N, 1230.'43" E"; "Muséum Paris, 1098 m; 30 Oct. 2010; D. Ouvrard Rec." "Muséum Paris, MNHN (EH) 24805 to 24807” • 17 ठ̊; "Philippines,

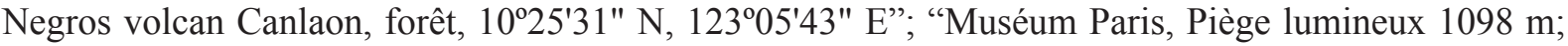
30 Oct. 2010; A. Soulier-Perkins rec.", "Muséum Paris, MNHN (EH) 24661, 24872 to 24886, 24808" •

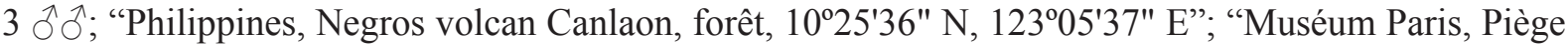
lumineux 1057 m; 31 Oct. 2010; D. Ouvrard Rec." "Muséum Paris, MNHN (EH) 24900 to 24902"

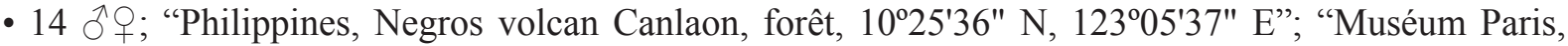
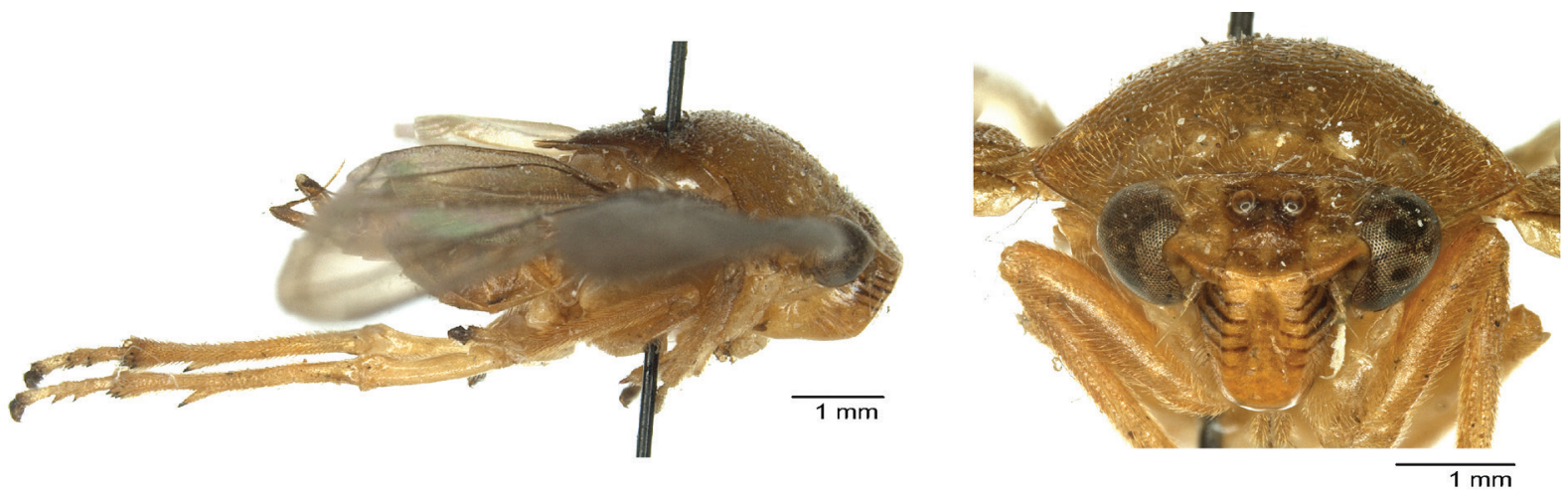

A

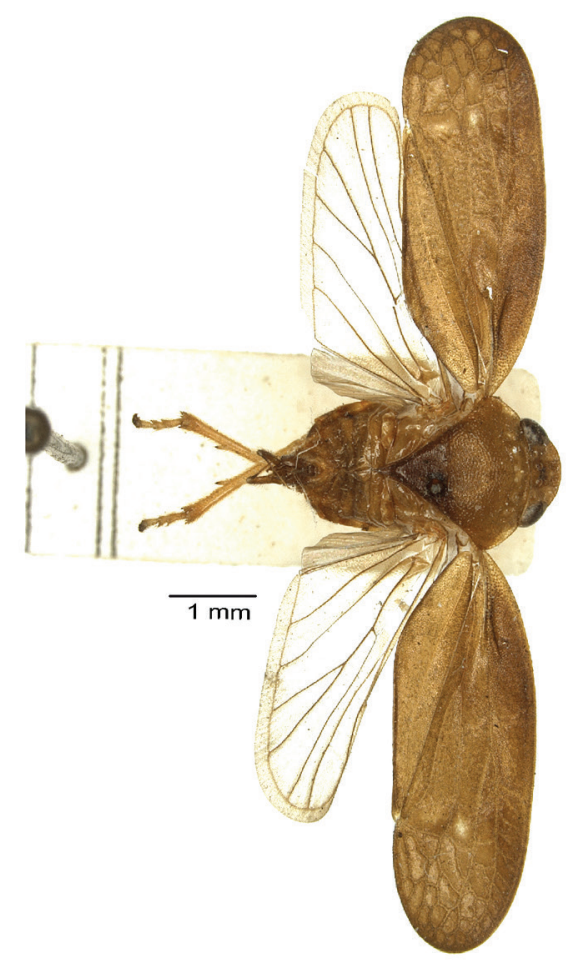

B

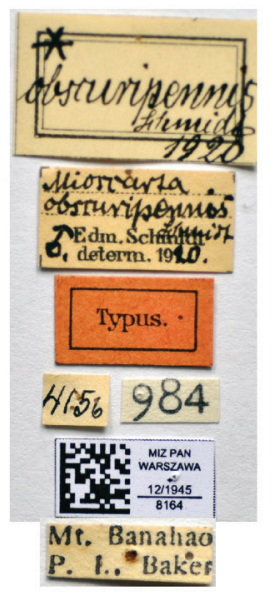

D

Fig. 9. Mioscarta obscuripennis Schmidt, 1920, ô holotype habitus. A. Lateral view. B. Frontal view. C. Dorsal view. D. Labels E. 
Table 4. Taxa included in the phylogeny with their voucher code, geographical origin and GenBank accession number for each marker used. ${ }^{a}$ Isolates; ${ }^{b}$ From GenBank; - Indicating absence of sequence.

\begin{tabular}{|c|c|c|c|c|c|c|}
\hline \multirow[b]{2}{*}{ Taxa } & \multirow{2}{*}{$\begin{array}{l}\text { Voucher } \\
\text { Code }\end{array}$} & \multirow{2}{*}{$\begin{array}{l}\text { Geographical } \\
\text { origin }\end{array}$} & \multicolumn{4}{|c|}{ GenBank Acession Number } \\
\hline & & & H3 & $\mathrm{CO}$ & & $18 \mathrm{~S}$ \\
\hline \multicolumn{7}{|c|}{ Ingroup (Cercopidae) } \\
\hline $\begin{array}{l}\text { Eoscarta } \\
\text { philippinica }\end{array}$ & $\mathrm{C}-00060^{\mathrm{a}}$ & Philippines & OK266891 & OK094471 & - & OK111059 \\
\hline Eoscarta borealis & C- $-00105^{\mathrm{a}}$ & Thailand & OK266892 & - & OK111085 & OK111060 \\
\hline Eoscarta borealis & C- $-00087^{\mathrm{a}}$ & Vietnam & OK266893 & OK094467 & - & OK111062 \\
\hline Eoscarta & C- $-00106^{a}$ & Thailand & - & OK094472 & OK111086 & OK111061 \\
\hline $\begin{array}{l}\text { liternoides } \\
\text { Jacobsoniella } \\
\text { bakeri }\end{array}$ & $C-00008^{a}$ & Philippines & OK266894 & OK094466 & OK111087 & OK111063 \\
\hline $\begin{array}{l}\text { Mioscarta } \\
\text { ferruginea }\end{array}$ & $\mathrm{C}-00027^{\mathrm{a}}$ & Philippines & - & - & OK111088 & OK111064 \\
\hline Mioscarta semperi & $\mathrm{C}-00017^{\mathrm{a}}$ & Philippines & - & - & OK111090 & OK111066 \\
\hline Mioscarta semperi & $\mathrm{C}-00022^{\mathrm{a}}$ & Philippines & - & OK094469 & OK111091 & OK111067 \\
\hline Mioscarta & $\mathrm{C}-00166^{\mathrm{a}}$ & Philippines & - & - & OK111089 & OK111065 \\
\hline $\begin{array}{l}\text { obscuripennis } \\
\text { Poeciloterpa } \\
\text { altissima }\end{array}$ & C- $-00042^{\mathrm{a}}$ & Philippines & OK266895 & - & OK111092 & OK111068 \\
\hline Poeciloterpa atra & C- $-00023^{a}$ & Philippines & - & OK094465 & OK111093 & OK111069 \\
\hline Poeciloterpa & $\mathrm{C}-00026^{\mathrm{a}}$ & Philippines & - & OK120386 & OK111094 & OK111070 \\
\hline $\begin{array}{l}\text { latipennis } \\
\text { Poeciloterpa } \\
\text { mangkas }\end{array}$ & $\mathrm{C}-00012^{\mathrm{a}}$ & Philippines & - & OK094470 & OK111095 & OK111071 \\
\hline $\begin{array}{l}\text { Poeciloterpa } \\
\text { minuta }\end{array}$ & C- $-00038^{\mathrm{a}}$ & Philippines & - & - & OK111096 & OK111072 \\
\hline $\begin{array}{l}\text { minuta } \\
\text { Trigonoschema } \\
\text { manoborum }\end{array}$ & $\mathrm{C}-00148^{\mathrm{a}}$ & Philippines & - & OK094468 & OK111097 & OK111073 \\
\hline Trigonoschema & $C-00167^{\mathrm{a}}$ & Philippines & - & OK094463 & OK111098 & OK111074 \\
\hline $\begin{array}{l}\text { Trigonoschema } \\
\text { rubercella }\end{array}$ & $\mathrm{C}-00075^{\mathrm{a}}$ & Philippines & - & OK094464 & OK111099 & OK111075 \\
\hline Wawi mehi & $\mathrm{C}-00095^{\mathrm{a}}$ & Papua New Guinea & OK266896 & - & OK111100 & OK111076 \\
\hline \multicolumn{7}{|c|}{ Outgroups Aphroporidae } \\
\hline Aphrophora alni & C-00190 a & $\mathrm{F}_{1}$ & 266889 & OK094476 & OK111082 & OK111056 \\
\hline Aphropl & $\mathrm{C}-00161^{\mathrm{a}}$ & & 266890 & - & OK111 & OK111057 \\
\hline Clovia $\mathrm{s}$ & C- $-00004^{\mathrm{a}}$ & Philippines & OK266887 & OK094478 & OK111080 & OK111054 \\
\hline Clovia $\mathrm{s}$ & $\mathrm{C}-00005^{\mathrm{a}}$ & Philippir & OK266888 & OK094477 & OK111 & OK111055 \\
\hline Perinoia sp. & $\mathrm{C}-000$ & & OK266884 & OK094474 & OK111 & OK111051 \\
\hline Perinoia sp. & $\mathrm{C}-00002^{\mathrm{a}}$ & Philippines & OK266885 & OK094475 & OK111078 & OK111052 \\
\hline Perinoia sp. & $C-00003^{\mathrm{a}}$ & Philippines & OK266886 & OK094473 & OK111079 & OK111053 \\
\hline \multicolumn{7}{|l|}{ Clastopteridae } \\
\hline Clastoptera & $01-07-18-58^{b}$ & USA & AY744862 & - & AY744790 & AY744824 \\
\hline $\begin{array}{l}\text { brunnea } \\
\text { Clastoptera }\end{array}$ & $01-07-15-06^{b}$ & USA & GU447121 & - & GU446795 & GU446885 \\
\hline $\begin{array}{l}\text { xanthocephala } \\
\text { Iba sp. }\end{array}$ & $C-00013^{a}$ & Philippines & - & OK094479 & OK111084 & OK111058 \\
\hline \multicolumn{7}{|l|}{ Machaerotidae } \\
\hline $\begin{array}{l}\text { Apomachaerota } \\
\text { reticulata }\end{array}$ & $06-02-15-17^{b}$ & Malaysia & GU447197 & - & GU446882 & GU446977 \\
\hline $\begin{array}{l}\text { Grypomachaerota } \\
\text { tyrhinata }\end{array}$ & $06-02-15-19^{b}$ & Malaysia & GU447198 & - & GU446883 & GU446978 \\
\hline Machaerota & $04-05-11-45^{\mathrm{b}}$ & Australia & - & - & GU446815 & GU446906 \\
\hline $\begin{array}{l}\text { pugionata } \\
\text { Machaerota } \\
\text { takeuchii }\end{array}$ & $04-10-15-80^{b}$ & Japan & - & - & GU446828 & GU446918 \\
\hline
\end{tabular}


CRISPOLON E. Jr. S. et al., New genus and new species of spittlebugs from the Philippines

Piège lumineux 1057 m; 31 Oct. 2010; A. Soulier-Perkins rec.", "Muséum Paris, MNHN (EH) 23651,

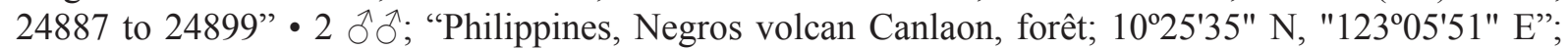
"Muséum Paris, 1117 m; 2 Nov. 2010; D. Ouvrard Rec.", "Muséum Paris, MNHN (EH) 24810 to 24811" - đ̂; "Philippines, Negros volcan Canlaon, forêt; 10²5'35" N, "12305'51" E"; "Muséum Paris, 1117 m; 2 Nov. 2010; A. Soulier-Perkins rec.", "Muséum Paris, MNHN (EH) 24812" • ○’; "Philippines, Negros volcan Canlaon, milieu anthropisé; $10^{\circ} 25^{\prime} 23^{\prime \prime} \mathrm{N}, 123^{\circ} 04^{\prime} 46^{\prime}$ E"; "Muséum Paris, Piège lumineux $745 \mathrm{~m}$;

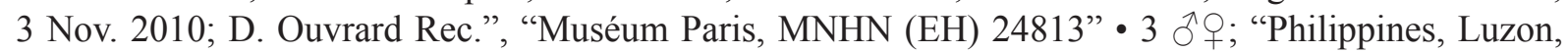
mount Isarog, Light trap"”, "Muséum Paris; 31 May 2011; S. Yap Rec.”, "Muséum Paris, MNHN (EH) 23649,24803 to $24804 ”$.

\section{Description}

MALE TERMINALIA. In lateral view, posterior margin of pygofer hump shaped in middle, last third abruptly truncated (Fig. 8E). Subgenital plates (Fig. 8F) very long relative to height of pygofer with fine tapering appendage longer than main plate, dorsal margin regularly convex and slightly curving on basal part, ventral margin roughly straight. Sterno-lateral plate present and roughly bean-shaped. Intermediate plate present, square shaped with a corner truncated, linking internal sides of lateral plate and subgenital plate. Paramere (Fig. 8G) dorsal margin rounded then regularly curving up to apex. Lateral margin with spine located subapically pointing dorsally, ventral margin rounded then sharply angled subapically pointing ventrally, apex without any spines. Aedeagus (Fig. 8H) with basal third of dorsal margin regularly bent, basal half enlarged then largely narrowed in the middle and gradually enlarging up to apex, roughly C-shaped, ventral margin regularly curved, apical extension pointing posteriorly, posterior protrusion axe-shaped with long thin extension prolonging the edge ventrally and slightly curving, postero-dorsal protrusion absent.

\section{Type Locality}

Philippines: Luzon: Mount Banahao, Quezon Province.

\section{Distribution}

Philippines: Luzon Island, Baguio, Quezon province, Mountain province; Visayas, Negros Island.

\section{Remarks}

Schmidt (1920) described this species from a single male specimen with few details on the male terminalia. Here, we provide, a detailed illustration and description of male terminalia from identified specimens using Schmidt description (Fig. 8), along with photographs of the male holotype and its labels (Fig. 9). The coloration of the specimen we identified and the holotype are different but the specimen from which we illustrated the male terminalia (Fig. 8) has a brighter coloration and is the closest to the description given by Schmidt while the holotype has a faded coloration.

Mioscarta semperi Jacobi, 1905

Fig. 10

Mioscarta semperi Jacobi, 1905: 21.

Mioscarta semperi - Schmidt 1909: 239-240. — Lallemand 1912: 117; 1949: 84-85. — Jacobi 1927: 549.

\section{Material examined}

PHILIPPINES • P; "Samar Calbayog Elev. - Sea level; 8 May 1950; R. Araneta Jr.", "Eoscarta sp. Bal '53" "UPLBMNH HEM-04054" • đ̃; " Calambog Ala Valley Elev.; 8 June 1950 S;. M Cendaña" 


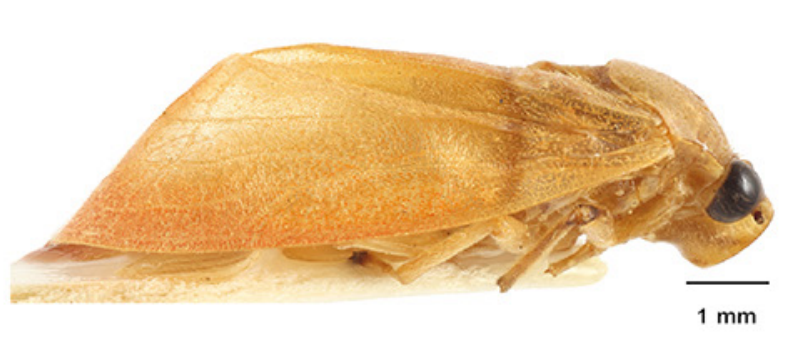

A
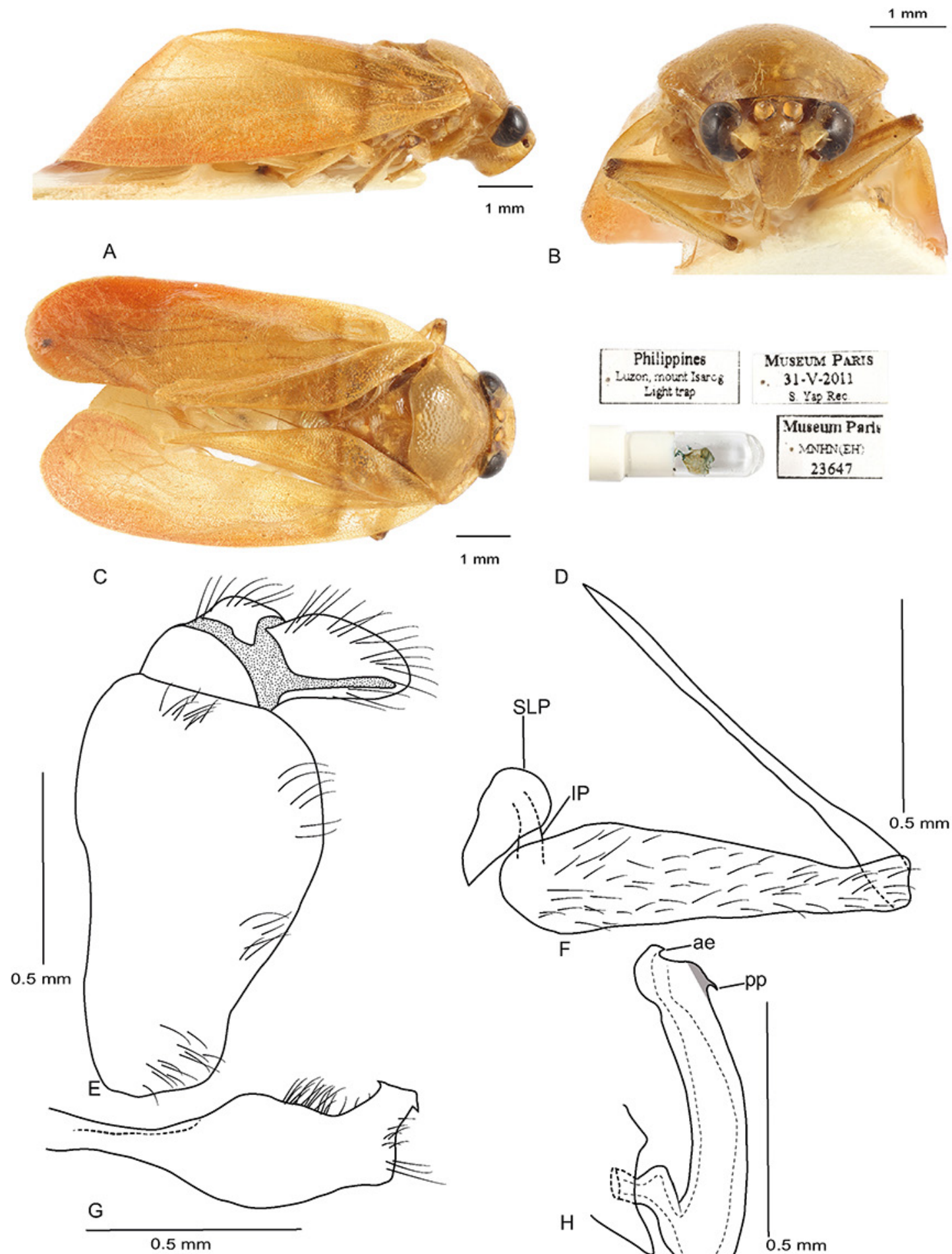

$1 \mathrm{~mm} \quad \mathrm{D}$

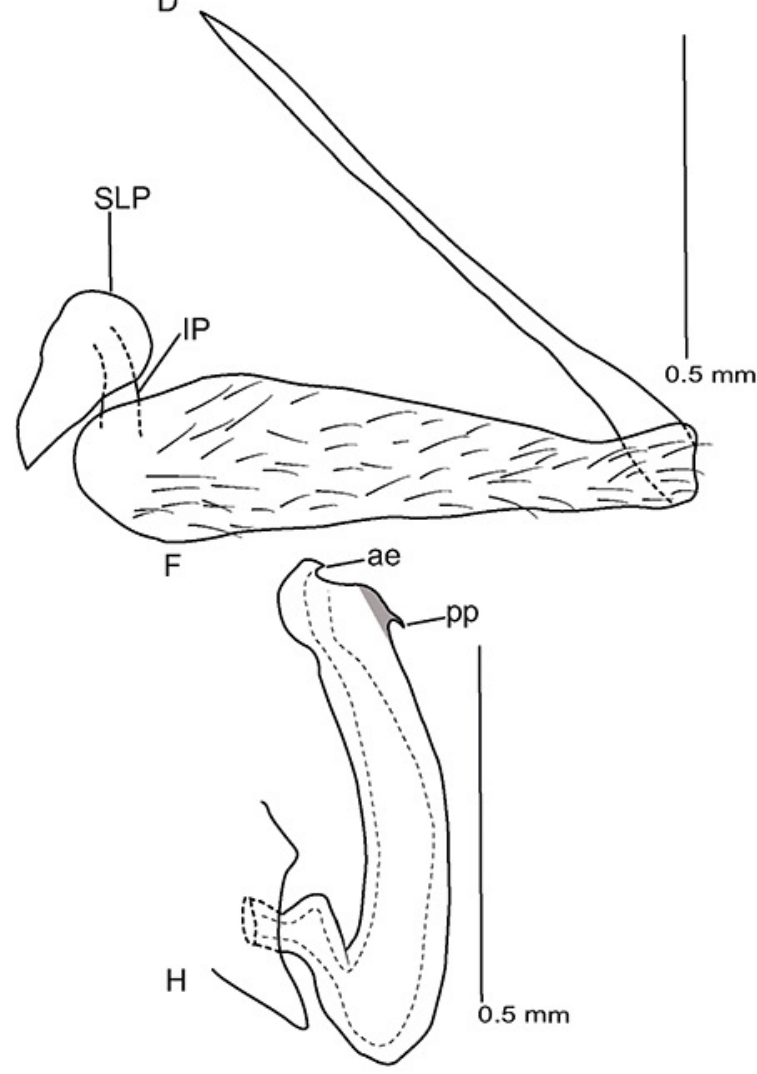

Fig. 10. Mioscarta semperi Jacobi, 1905 habitus and $\widehat{\partial}$ terminalia in lateral view. A. Lateral view. B. Frontal view. C. Dorsal view. D. Labels. E. Pygofer and anal tube. F. Sterno-lateral, intermediate and subgenital plate. G. View paramere. H. View aedeagus. 
CRISPOLON E. Jr. S. et al., New genus and new species of spittlebugs from the Philippines

“Eoscarta sp.1”" “UPLBMNH HEM-04061" • 우; "BUKIDNON Impalitaw BFD; 19 Nov. 1988; VPG"

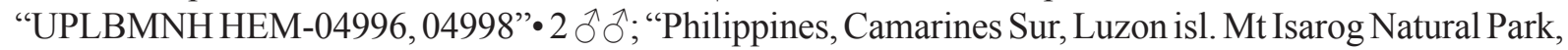
Panicuason Naga", "Muséum Paris; 1 May 2011; S.A. Yap, M.V. Yngente, O.L. Eusebio rec.", "Muséum

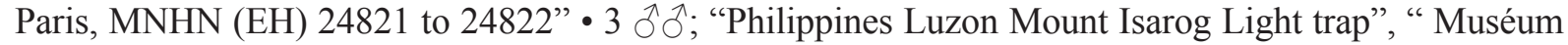
Paris; 31 May 2011; S. Yap Rec", "Muséum Paris, MNHN (EH) 23647, 24823 to 24824” • ; " "PH. MT. Makiling Los Baños, Laguna Luz; 21 Nov. 2016; WS HWANG", "séquençage par Elorde Crispolon C-00085", "Muséum Paris, MNHN (EH) 24817" • o; "Brgy. Irosin, Sorsogon; 2 Feb. 2017; Coll. AK AMARGA"; "Muséum Paris, MNHN (EH) 24818" • +; "Ph. Brgy Perez, Kidapawan City; 5 May 2017; C.S. Satohito", "ECpc 057", "Ph. Mt. Akir Akir, Pigcawayan, North Cotabato; 8-9 Sept. 2018; G. Biacolo, K.C. Crispolon \& R. Guillermo"; "séquençage par Elorde Crispolon C-00017", "Muséum Paris, MNHN (EH) 24819", "Ph. Brgy Kidama, Matalam, North Cotabato; 14 Mar. 2019; K.C. Crispolon", "séquençage par Elorde Crispolon C-00022", "Muséum Paris, MNHN (EH) 24820”.

\section{Description}

Male terminalia. In lateral view, posterior margin of pygofer regularly convex last third slightly curved (Fig. 10E). Subgenital plates (Fig. 10F) very long relative to height of pygofer with fine tapering appendage slightly longer than main plate, dorsal margin of plate regularly rounded, ventral margin straight. Sterno-lateral plate present, slightly elongated. Intermediate plate present, cylindrical, linking internal side of lateral and subgenital plates. Paramere (Fig. 10G) not globose, dorsal margin rounded and regularly curving, apex with a sharp spine pointing antero-dorsally, ventral margin regularly rounded then subapical part largely rounded, protruding and finishing with a sharp spine pointing ventrally. Aedeagus (Fig. 10H) with basal third of dorsal margin angled and sharply bent in acute angle and last $2 / 3$ vertical and roughly C-shaped, slightly humped in apical part of dorsal margin before apical extension, apical extension pointing posteriorly, posterior protrusion thin very short and spine-shaped, postero-dorsal protrusion absent.

\section{Type Locality}

Philippines: Philippine Islands.

\section{Distribution}

Philippines: Luzon, Visayas and Mindanao Islands.

\section{Remarks}

According to what Jacobi (1905) mentioned at the end of the description "Mus. Berol. Nr. 9107: Semper coll., 2 Ex.". He examined two specimens and described this species from atleast one female without a description of the male terminalia. We illustrate and describe them here based on a specimen identified using Lallemand's (1949) key.

Mioscarta translucida Crispolon \& Yap sp. nov. urn:lsid:zoobank.org:act:7D16117A-699D-4C7E-B037-E290B274EA8C

Fig. 11

\section{Diagnosis}

M. translucida is the only species with tegmen translucid without any other coloration.

\section{Etymology}

The species name refers to the translucid tegmen and is based on the latin word "translucidus" which means allowing light to pass through. 


\section{Material examined}

Holotype

PHILIPPINES • đ; "Philippines, Camarines Sur, Luzon isl. Mt Isarog Natural Park, Panicuason Naga", "Muséum Paris; 1 May 2011; S.A. Yap, M.V. Yngente \& O.L. Eusebio rec."; "Muséum Paris, MNHN (EH) 24749"; MNHN.

\section{Description}

BoDy. Length $10 \mathrm{~mm}$ (tegmina included), width $4 \mathrm{~mm}$.

HEAD (Figs 1A, 11B). In dorsal view, large ocelli, distance between eyes less than 8 times ocellus diameter, distance between ocelli equals one ocellus diameter, distance between ocellus and the compound eye 2 times ocellus diameter, ocelli closer to each other than from compound eyes. Eyes not prominent, length 1.44 times than wide. Vertex and frons longitudinal median carina absent. Vertex as long as wide with 3 times ocellus diameter in between two vertex grooves outside ocelli and 3 times ocellus diameter between anterior and posterior vertex margins. Postclypeus with longitudinal furrow, slightly swollen and ovoid shape in frontal view, widest part at mid height (Figs 1A, 11B), not receding and prior to anteclypeus where it bends forming right angle in lateral view (Fig. 11A). Rostrum long, surpassing mesocoxae. Thorax (Figs 2A, 11A-C). In dorsal view, pronotum with anterior concavities on each side, anterior margin as wide as posterior margin of head including eyes, anterolateral margins curved, posterior margin grooved, postero-lateral margins slightly concave, longer than anterolateral margins, humeral angle rounded. In lateral view, pronotum curving not more than $25^{\circ}$ (Figs 2, 11A) Scutellum as long as wide with large median dimple (Fig. 11C). Tegmen (Fig. 11A-C). R bifurcates on apical half, $\mathrm{M}$ bifurcate on basal third, apical reticulation not well developed without concave apical cells. Posterior wing (Fig. 3A). Rp separating from SC+Ra nearly at midlength, $\mathrm{M}$ reaches ambient vein, Cua and Cup fused at base and $\mathrm{m}$-cu links $\mathrm{M}$ to Cua before Cua bifurcation, common base for Cup and Cua originate at base of wing, 7 longitudinal veins and 5 apical cells between SC+Ra and Cup, angular protrusion of costal margin near its base. Metafemur with apical spine in inner margin, metatibiae bearing 1 lateral spine.

MALE TERMINALIA. In lateral view, posterior margin of pygofer slightly undulating in the middle with slight curved on the last third (Fig. 11E). Subgenital plates (Fig. 11F) dorsal and ventral margin of main plate roughly straight, sterno-lateral plate present, slightly elongated, intermediate plate present, elongated slightly triangular shaped, linking internal sides of lateral and subgenital plates. Paramere (Fig. 11G) not globose, dorsal margin convex and regularly curving finishing with rounded apex with very minute groove, ventral margin convex, apex with spiniform process pointing antero-ventrally. Dorsal and ventral margins of aedeagus undulating. Aedeagus (Fig. 11H) with basal third of dorsal margin regularly bent without angle before the bent part and last $2 / 3$ vertical and S-shaped, ventral margin regularly curved then slightly concaved before the base, apical extension sharp pointing postero-ventrally, posterior protrusion sharp at the apex hook-shaped, postero-dorsal protrusion absent.

Color. Head and pronotum brown with yellow patches, rostrum yellowish white, pedicel of antenna brown, legs yellowish and abdomen light brown. Tegmen partially translucid, opaque parts being yellowish with darker patches.

\section{Type Locality}

Philippines: Luzon, Camarines Sur, Mount Isarog Natural Park.

\section{Distribution}

Philippines: Luzon Island. 


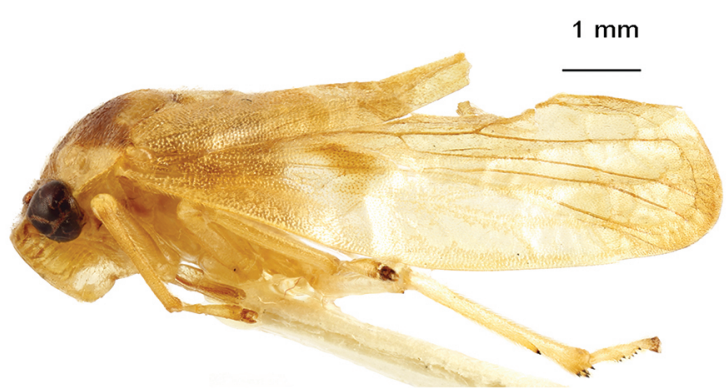

A

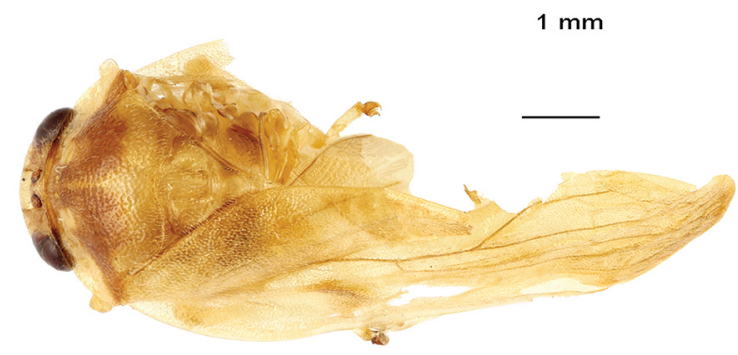

B
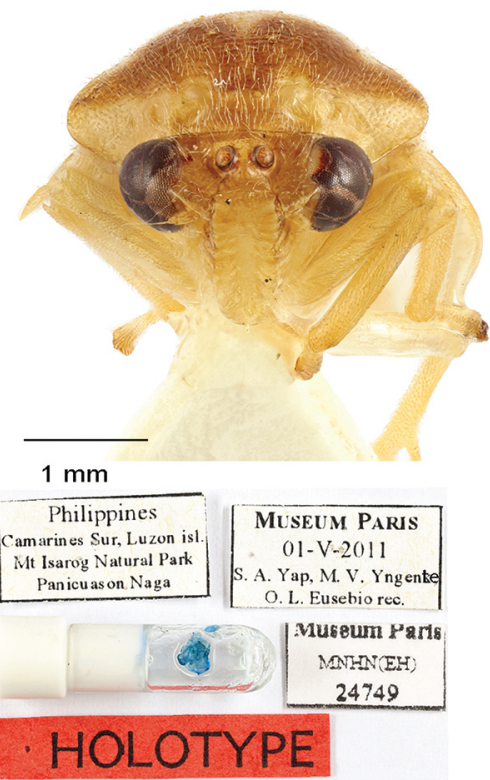

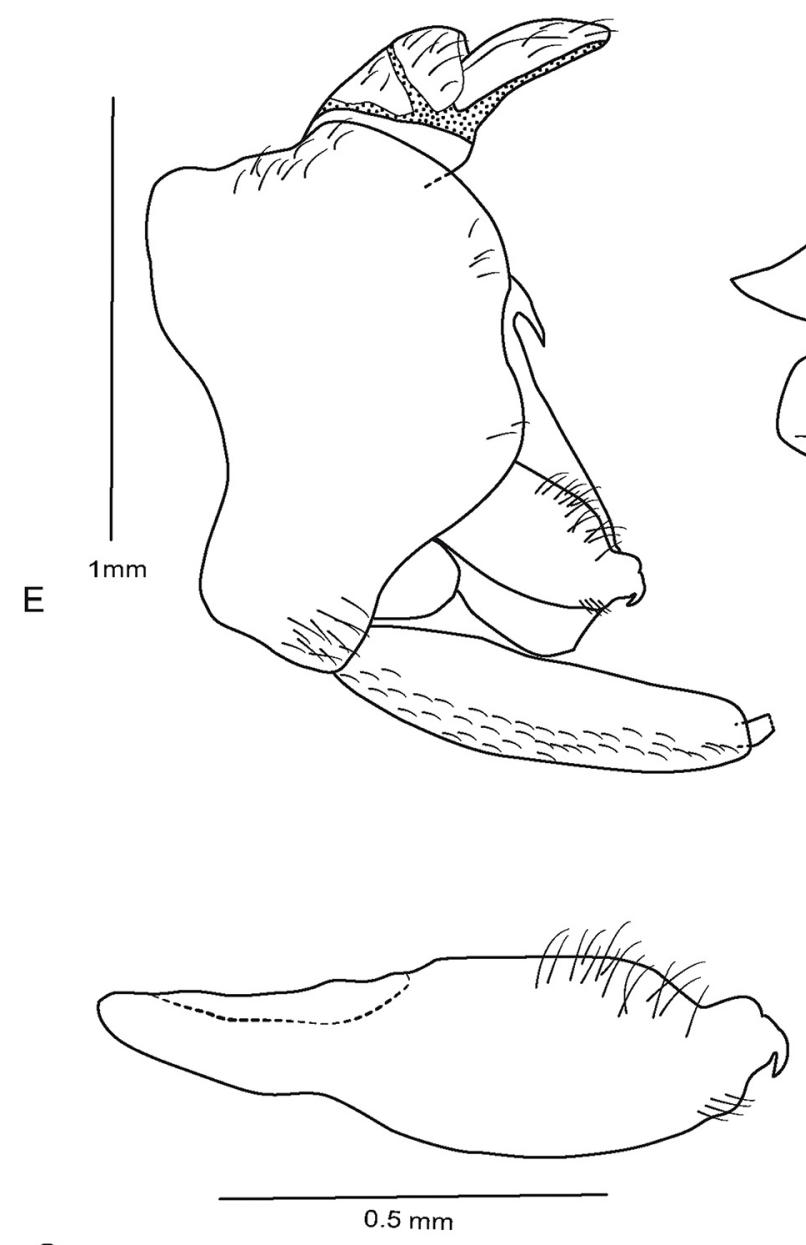

G

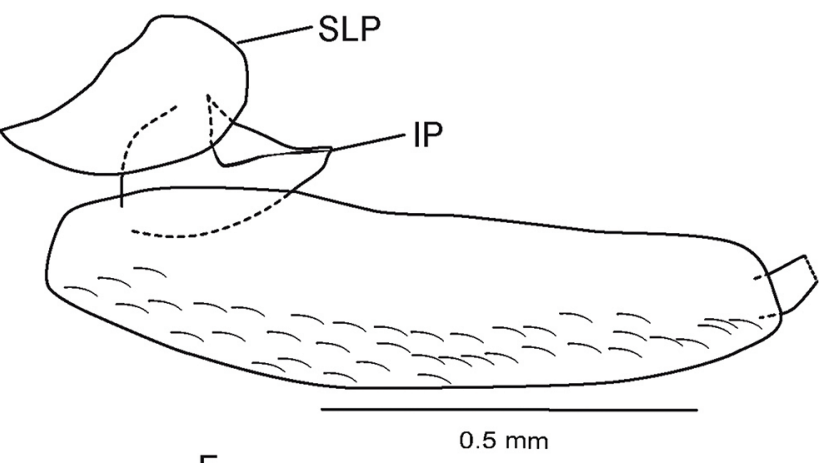

$\mathrm{F}$

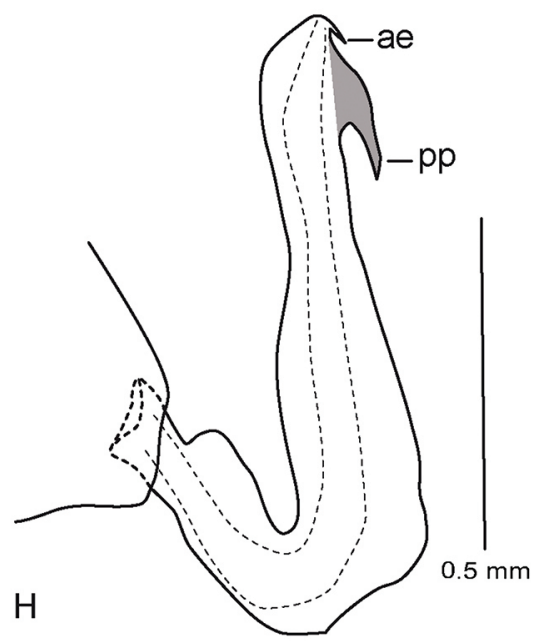

Fig. 11. Mioscarta translucida Crispolon \& Yap sp. nov. holotype, habitus and $\widehat{\partial}$ terminalia in lateral view. A. Lateral view. B. Frontal view. C. Dorsal view. D. Labels. E. Terminalia. F. Sterno-lateral, intermediate and subgenital plates. G. Paramere. H. Aedeagus. 


\section{Remarks}

The exact length of subgenital plate relative to height of pygofer is not provided because part of the appendage is damaged.

Trigonoschema Crispolon \& Soulier-Perkins gen. nov. urn:1sid:zoobank.org:act:C8299091-60D3-49B4-852F-2145956D7FE9

Figs 12-16

\section{Type species}

Trigonoschema manoborum sp. nov. here designated.

\section{Diagnosis}

In dorsal view, teardrop shaped and apex of tegmen not visible due to folding. In lateral view, very steeply declivous pronotum and crown of the head, nearly form a right angle with the rest of the dorsum in profile.

\section{Etymology}

When observed in dorsal view, the general shape of the habitus fits in a triangle even if a closer observation would lead to describe it as teardrop shaped. The name is built using two greek words "trigonos" for triangular and "schema" for shape. It is neutral.

\section{Description}

Body. Length 9.5-12.5 mm (tegmen included), width 4-5 mm.

GENERAL SHAPE. Not dorso-ventrally flattened, in lateral view total length around 3 times height (Figs 2B, 12-16A). Ocelli small, distance between eyes 9-10.5 times ocellus diameter (Figs 1, 12-16B). Distance between ocellus and compound eye less than 4 times ocellus diameter ((Figs 1, 12-16B). Distance between ocelli 1.5 ocellus diameter (Figs 1, 12-16B). Postclypeus with longitudinal furrow, swollen laterally and slightly ovoid shape in frontal view, widest part close to frons, not receding and prior to anteclypeus where it bends rounded in lateral view, with wide longitudinal furrow (Figs 1, 12-16B). Pronotum curving around $45^{\circ}$ angle (Figs 2B, 12-16A). Tegmen translucid without concave apical cells. Apical curve of tegmen rarely visible in dorsal view, tegmen folded in such a way that widest part of habitus is before midlength of tegmen (Figs 12-16C).

MALE TERMinAlia. Subgenital plates without fine appendage (Fig. 12F), if present shorter than main plate, not forming an acute angle shape with the main plate (Figs 14, 16F). Paramere globose, lateral margin of paramere with protrusion, on the ventral margin on the sub apex with angular protrusion ornamented with set of setae (Figs 12, 14, 16G). Pp of aedeagus thick and beak-like shape (Figs 12, 14, 16H).

\section{Distribution}

Philippines.

\section{Key to the species of Philippine Trigonoschema gen. nov.}

1. Most of vertex and frons bright yellow and postclypeus orange (Figs 12-13)

T. manoborum Crispolon \& Soulier-Perkins sp. nov.

- Vertex, frons and postclypeus of the same color that can be brown, orange or red (Figs 14-16) ..... 2

2. Red band running across base of tegmen and scutellum (Fig. 16) T. rubercella Crispolon \& Guilbert sp. nov. 
CRISPOLON E. Jr. S. et al., New genus and new species of spittlebugs from the Philippines

- $\quad$ No red band visible in dorsal view (Figs 12-13)...................................................................... 3

3. Scutellum yellow (Fig. 14) ................................................. negrosensis Crispolon \& Yap sp. nov.

- Scutellum brown (Fig. 15) ................................................... pallida (Lallemand, 1927) comb. nov.

Trigonoschema manoborum Crispolon \& Soulier-Perkins gen. et sp. nov. urn:lsid:zoobank.org:act:EDF12AD9-920E-46AA-937F-D66CE3ACD6B1

Figs $12-13$

\section{Diagnosis}

Tegmen, white in basal $1 / 4$ with orange marking on the basal half of the margin of the clavus. Scutellum orange with yellowish white coloration in middle. Most of vertex and frons bright yellow and postclypeus dull orange for male and bright orange for female.

\section{Etymology}

Manoborum, genitive plural made from Manobos. The Manobo tribe is found in Bongolanon, Magpet, North Cotabato.

\section{Material examined}

Holotype

PHILIPPINES • đ̊; "Philippines, Mt. Apo, Bongolanon, Magpet N. Cotabato"; "5 May 2018; Daniela Alcalde"; "holotype", "séquençage par Elorde Crispolon C-00148", "Muséum Paris, MNHN (EH) 24871"; MNHN.

\section{Paratype}

PHILIPPINES • १; "PH. MT. APO, Bongolanon, Magpet, Cot, Mindanao, 17 Dec. 2015, ES Crispolon" "séquençage par Elorde Crispolon C-00078" "UPLBMNH HEM-05054"; UPLBMNH.

\section{Description}

Body Length. $10.5 \mathrm{~mm}$ (tegmina included), width $4 \mathrm{~mm}$.

HeAD (Figs 1, 12-13B). In dorsal view, ocelli small, distance between eyes 10.5 times ocellus diameter (Figs 1, 12-13B). Distance between ocellus and compound eye less than 3.5 times ocellus diameter ((Figs 1, 12-13B). Distance between ocelli 1.5 ocellus diameter (Figs 1, 12-13B). Eyes not prominent, length 1.15 times than wide. Vertex with longitudinal short median carina, slightly longer than wide. Frons without any carina. Postclypeus with dimple below margin of frons and a longitudinal furrow clearly marked in male, swollen laterally and slightly ovoid shape in frontal view, widest part close to frons, not receding and prior to anteclypeus where it bends rounded in lateral view (Figs 1B, 12-13A). Rostrum long, reaching but not surpassing mesocoxae. Thorax (Figs 2B, 12-13A-C). In dorsal view, pronotum with anterior deep concavities on each side, much clearly marked in female, longitudinal median carina absent. Anterior margin of pronotum as wide as posterior margin of head including eyes, anterolateral margins curved, posterior margin grooved, postero-lateral margins slightly concave, slightly longer than anterolateral margins, humeral angle rounded. In lateral view, pronotum angle around $45^{\circ}$ (Figs $2 \mathrm{~B}, 12-13 \mathrm{~A}$ ), scutellum as long as wide with large median dimple (Figs 12-13C). Tegmen (Figs 12-13A-C). R bifurcates on apical half, M bifurcate on basal third, apical reticulation not well developed without concave apical cells. Posterior wing (Fig. 13E). $2 / 3$ of $\mathrm{M}$ length alone then fuses with Rp before reaching ambient vein, 6 longitudinal veins on posterior wing reaching apex, four apical cells between $\mathrm{SC}+\mathrm{Ra}$ and $\mathrm{Cup}$, angular projection near base of costal margin present. Legs. Metafemur with apical spine in inner margin, metatibiae bearing 1 lateral spine. 


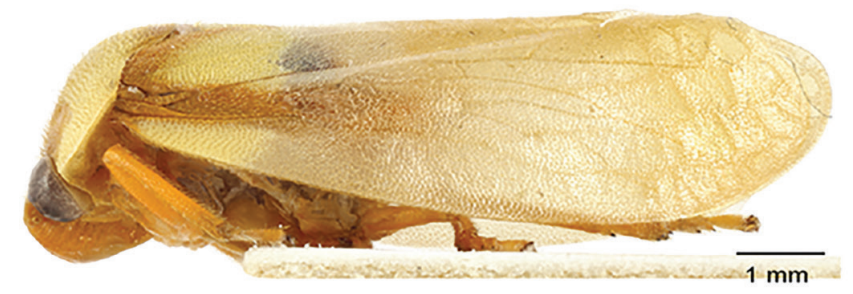

A
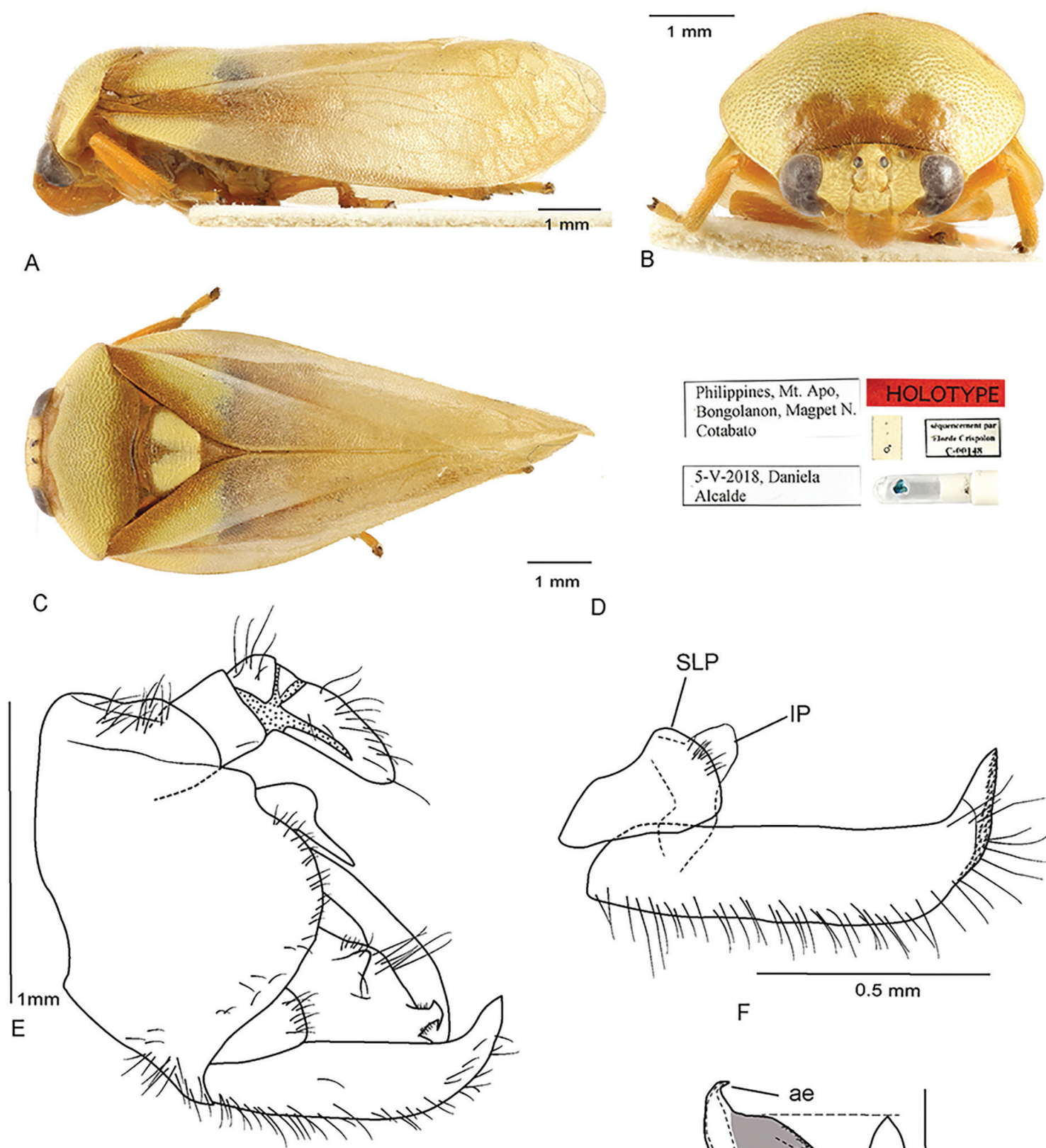

F

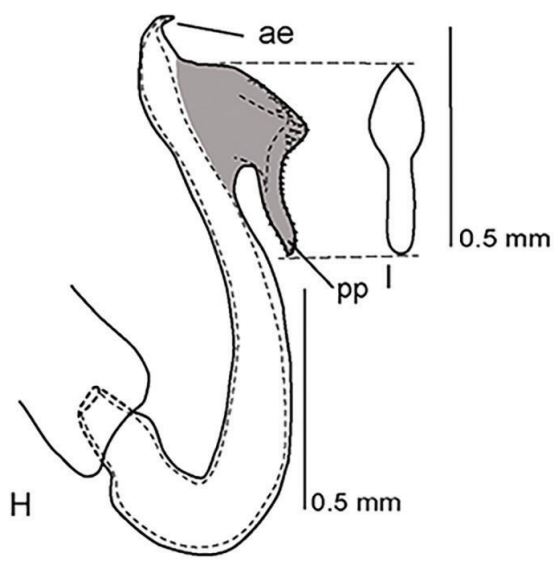

G

Fig. 12. Trigonoschema manoborum Crispolon \& Soulier-Perkins sp. nov. holotype, habitus and $\widehat{\partial}$ terminalia in lateral view. A. Lateral view. B. Frontal view. C. Dorsal view. D. Labels. E. Terminalia. F. Sterno-lateral, intermediate and subgenital plates. G. Paramere. H. Aedeagus. I. Shape of pp in posterior view. 
Male terminalia. In lateral view, posterior margin of pygofer (Fig. 12E) largely convex in middle getting concave in last third and presenting a bump in first third. Subgenital plates (Fig. 12F) with equal length relative to height of pygofer without any appendage, regularly tapering towards apex, dorsal margin straight then curving up towards apex, ventral margin straight half its length then regularly curving dorsally, minute denticuli covering last quarter toward apex. Sterno-lateral plate present,
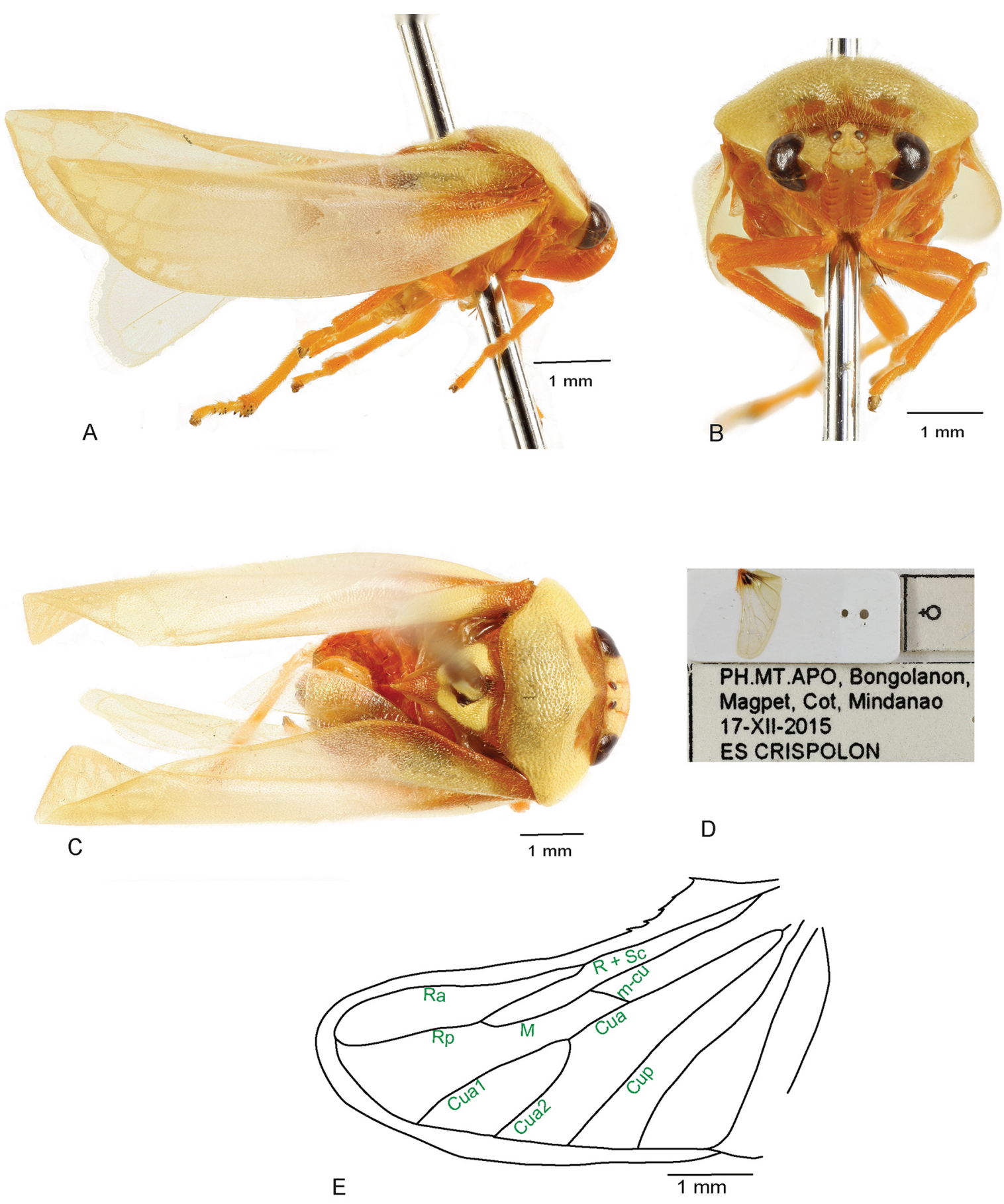

Fig. 13. Trigonoschema manoborum Crispolon \& Soulier-Perkins sp. nov., $\subsetneq$ p paratype (UPLBMNH HEM-05054). Habitus and posterior wing. A. Lateral view. B. Frontal view. C. Dorsal view. D. Labels. E. Posterior wing. 
slightly elongated. Intermediate plates present, elongated and linked with internal sides of lateral plate and subgenital plate. Paramere (Fig. 12G) globose, dorsal margin convex then curving regularly and finishing with a sharp spine pointed antero dorsally, lateral margin with process in middle part pointing postero-dorsally, ventral margin regularly rounded with subapical part largely protruding posteriorly then finishing with a sharp spine pointing ventrally. Aedeagus (Fig. 12H) with dorsal margin slightly humped at base then concave most its length, curving regularly half its length vertically and finishing $1 / 4$ its length convex in a C-shaped, apical extension pointing posteriorly, posterior protrusion thick, duck head shaped with minute denticuli along posterior margin, in posterior view basal half lobular (Fig. 12I), postero-dorsal protrusion absent.

CoLor. Head, and pronotum white-yellowish with orange markings, postclypeus, rostrum, abdomen and legs orange, pedicel of antennae yellowish brown, scutellum orange with yellowish white markings medially. Tegmen, translucid yellowish white, white in basal $1 / 4$ with orange marking in between white and on the basal half of the margin of the clavus, last $3 / 4$ yellowish getting yellowish brown in apical fourth.

\section{Type Locality}

Philippines, Mt. Apo, Bongolanon, Magpet North Cotabato.

\section{Distribution}

Philippines: Mindanao Island.

Trigonoschema negrosensis Crispolon \& Yap gen. et sp. nov. urn:lsid:zoobank.org:act:BFF10DEF-C172-4861-A9AE-81AA8B88657B

Fig. 14

\section{Diagnosis}

In general shape, T. negrosensis is similar to T. rubercella but their coloration is distinctly different. T. negrosensis in dorsal view presents a dirty yellow pronotum followed by a light orange scutellum framed by yellow irregular patches on clavus of tegmen while T. rubercella presents a bright yellow pronotum followed by a red band running on basal parts of tegmen and across scutellum.

\section{Etymology}

The species is named after the island where it was found: Negros.

\section{Material examined}

\section{Holotype}

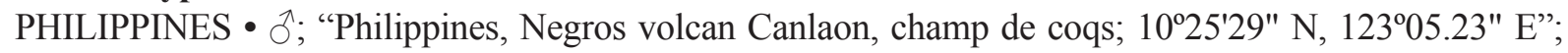
"Muséum Paris, Piège lumineux, 932 m; 28 Oct. 2010; A. Soulier-Perkins rec.", "UPLBMNH HEM05055"; UPLBMNH.

\section{Paratypes}

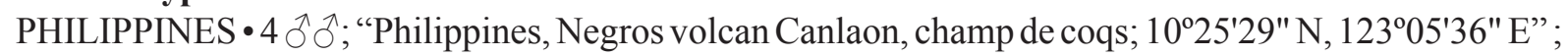
"Muséum Paris, 1050 m; 28 Oct. 2010; D. Ouvrard Rec.", "Muséum Paris, MNHN (EH) 23639,

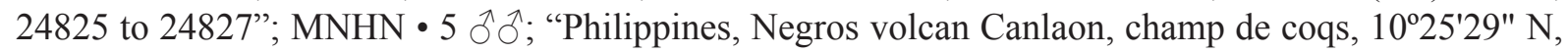
1230․ㄹ" E"; "Muséum Paris, Piège lumineux 932 m; 28 Oct. 2010; A. Soulier-Perkins rec.", "Muséum Paris, MNHN (EH) 24828", "Muséum Paris, MNHN (EH) 24829 to 24830", "séquençage par Elorde Crispolon, C-00076, C-00077", "Muséum Paris, MNHN (EH) 24831"; MNHN. "UPLBMNH HEM-

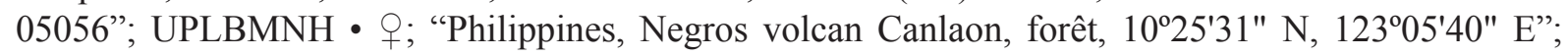




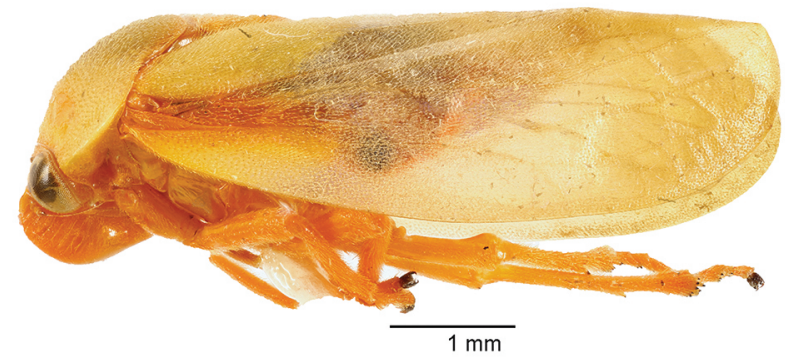

A

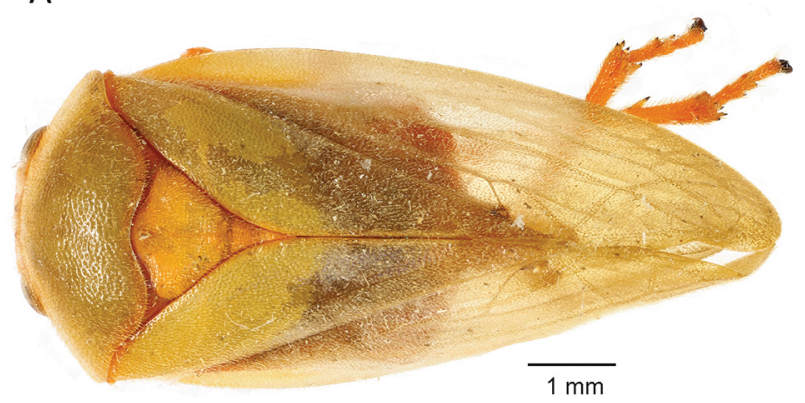

C
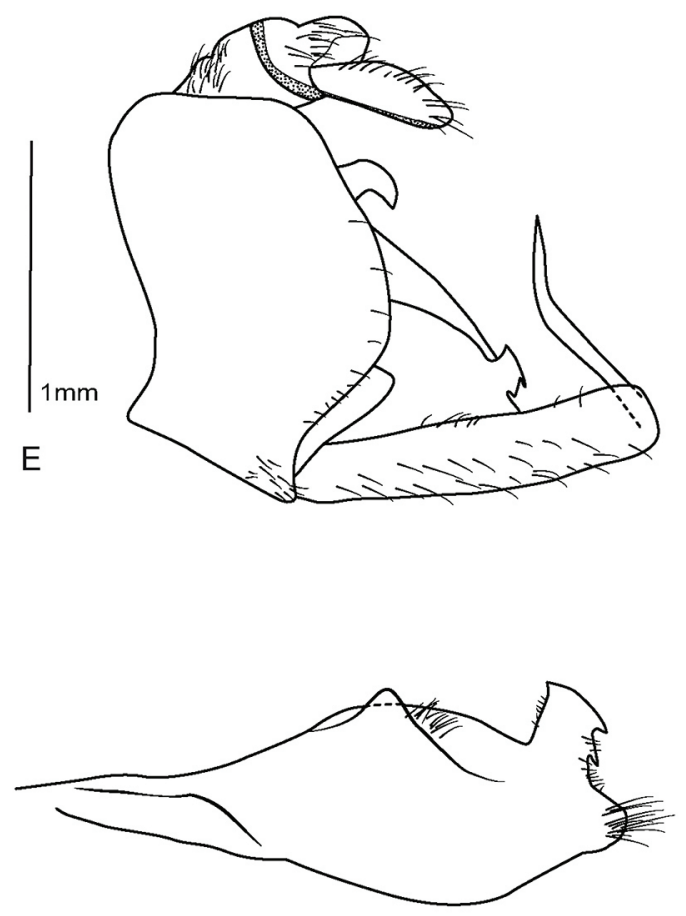

G

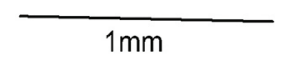

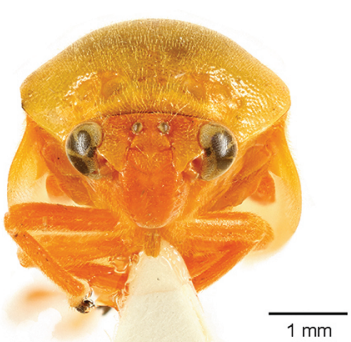

B

\begin{tabular}{|c|c|}
\hline 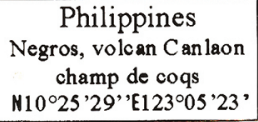 & $\begin{array}{c}\text { MUSEUM PARIS } \\
\text { Piège lumineux, } 932 \mathrm{~m} \\
28-\mathrm{X}-2010 \\
\text { A. Soulier-Perkins rec. }\end{array}$ \\
\hline
\end{tabular}

HOLOTYPE

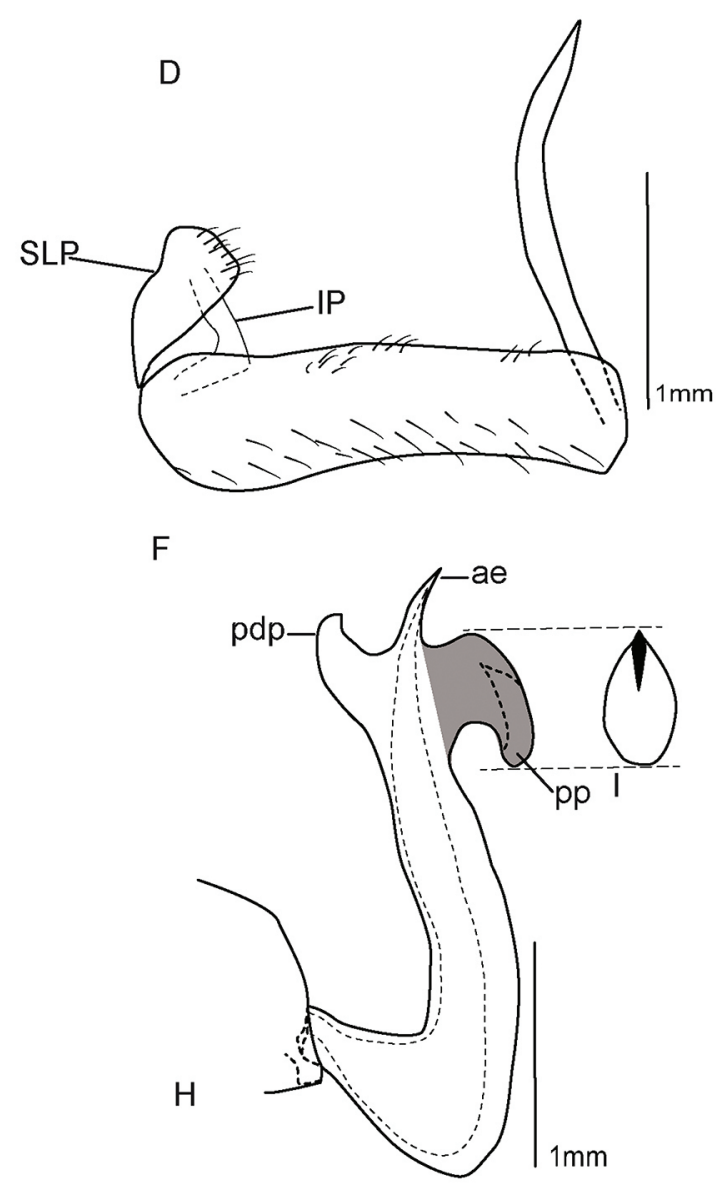

Fig. 14. Trigonoschema negrosensis Crispolon \& Yap sp. nov., holotype, habitus and $\widehat{\partial}$ terminalia in lateral view. A. Lateral view. B. Frontal view. C. Dorsal view. D. Labels. E. Terminalia. F. Sternolateral, intermediate and subgenital plates. G. Paramere. H. Aedeagus. I. Shape of pp in posterior view. 
"Muséum Paris, Piège lumineux 1098 m; 29 Oct. 2010; A. Soulier-Perkins rec.”, "Muséum Paris, MNHN (EH) 24832"; MNHN • O; "Philippines, Negros volcan Canlaon, forêt, $10^{\circ} 25^{\prime} 31$ " N, 1230.5'43" E" "Muséum Paris, 1098 m, 30 Oct. 2010, D. Ouvrard Rec.", "Muséum Paris, MNHN (EH) 24833"; MNHN

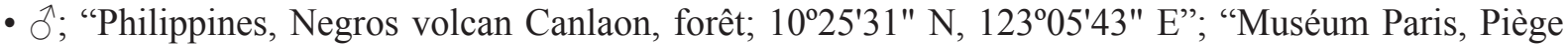
lumineux 1098 m; 30 Oct. 2010; A. Soulier-Perkins rec.", "Muséum Paris, MNHN (EH) 24834"; MNHN

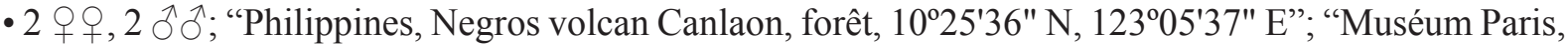
Piège lumineux 1057 m, 31 Oct. 2010; A. Soulier-Perkins rec.", "Muséum Paris, MNHN (EH) 24835 to 24837" "séquençage par Elorde Crispolon C-00167", "Muséum Paris, MNHN (EH) 24838"; MNHN

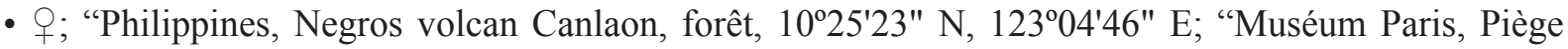
lumineux 745 m; 3 Nov. 2010; A. Soulier-Perkins rec.", "Muséum Paris, MNHN (EH) 24839"; MNHN.

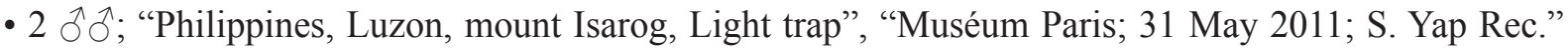
"Muséum Paris, MNHN (EH) 24840 to 24841"; MNHN.

\section{Description}

BoDy. Length 11-12.5 mm (tegmina included), width 4-5 mm.

HEAD (Figs 1, 14B). In dorsal view, ocelli small, distance between eyes 9.5 times ocellus diameter (Figs 1, 14B). Distance between ocellus and compound eye less than 3 times ocellus diameter (Figs 1, 14B). Distance between ocelli 1.5 ocellus diameter (Figs 1, 14B). Eyes not prominent, length 1.33 times than wide. Vertex with longitudinal median carina, as long as wide. Frons without median carina. Postclypeus with dimple below margin of frons and a longitudinal furrow clearly marked in males, swollen laterally and slightly ovoid shape in frontal view, widest part close to frons, not receding and prior to anteclypeus where it bends rounded in lateral view (Figs 1B, 14A). Rostrum long, reaching but not surpassing mesocoxae. Thorax (Figs 2B, 14A-C). In dorsal view, pronotum with anterior deep concavities on each side, much clearly marked in female, longitudinal median carina absent. Anterior margin of pronotum as wide as posterior margin of head including eyes, anterolateral margins curved, posterior margin grooved, postero-lateral margins slightly concave, slightly longer than anterolateral margins, humeral angle rounded. In lateral view, pronotum angle around $45^{\circ}$ (Figs $2 \mathrm{~B}, 14 \mathrm{~A}$ ). Scutellum as long as wide with large median dimple (Fig. 14C). Tegmen (Fig. 14A, C). R bifurcates on apical half, $\mathrm{M}$ bifurcate on basal third, apical reticulation not well developed without concave apical cells. Posterior wing (Fig. 3A). Rp separating from SC+Ra nearly at midlength, $\mathrm{M}$ reaches ambient vein, Cua and Cup fused at base and $\mathrm{m}$-cu links $\mathrm{M}$ to Cua before Cua bifurcation, 7 longitudinal veins and 5 apical cells between $\mathrm{SC}+\mathrm{Ra}$ and Cup, angular protrusion of costal margin near its base. Metafemur with apical spine in inner margin, metatibiae bearing 1 lateral spine.

MaLe terminalia. In lateral view, posterior margin of pygofer (Fig. 14E) convex in middle and clearly concave on last third. Subgenital plates (Fig. 14F) slightly longer relative to height of pygofer with fine appendage shorter forming acute angle with main plate, dorsal and ventral margin of main plate roughly straight. Sterno-lateral plate present and slightly elongated. Intermediate plates present, boomerangshaped, linking internal sides of lateral plate and subgenital plate. Paramere (Fig. 14G) globose, dorsal margin convex then curving regularly and finishing with a sharp spine pointed antero dorsally, lateral margin with process in middle part pointing dorsally, ventral margin regularly rounded with subapical part largely protruding posteriorly then finishing with two sharp spines pointing postero-ventrally. Aedeagus (Fig. 14H) with basal fourth almost straight then bending vertically and slightly wave-shape up to postero-dorsal protrusion, apical extension pointing dorsally, posterior protrusion thick, beakshaped, in posterior view, very slim at the top and petal shaped and foliaceous toward the base (Fig. 14I), postero-dorsal protrusion present.

CoLOR. Head including postclypeus and rostrum, legs and abdomen orange, pronotum orange white anterior half, greenish on apical part, scutellum greenish white with orange coloration, antennal scape and pedicel 
orange, flagellum with brown coloration. Tegmen translucid yellow, basal third with white coloration in costal margin and clavus and with orange coloration in between white coloration, last $2 / 3$ yellowish brown.

\section{Type Locality}

Philippines: Visayas, Negros Occidental, Mount Kanlaon.

\section{Distribution}

Philippines, Visayas, Negros Island; Luzon Island.

Trigonoschema pallida (Lallemand, 1927) comb. nov. Fig. 15

Mioscarta pallida Lallemand, 1927: 113.

Mioscarta pallida - Lallemand 1949: 84-85.

\section{Distribution}

Philippines: Philippine Islands.

\section{Remarks}

Lallemand in 1927 described this species based on the pattern of coloration and placed it in Mioscarta. He mentioned only a single male specimen (holotype), however, in the collection it is labelled as female (Fig. 15D). This species does not conform to Breddin's definition of Mioscarta but possesses characters of Trigonoschema gen. nov. For this reason we transfer it to this new genus. We could not borrow the holotype, however some photographs of the habitus and labels were kindly provided by Mick Webb (NHM). They are integrated here (Fig. 15) and are sufficient to see the most obvious characters of Trigonoschema gen. nov.

Trigonoschema rubercella Crispolon \& Guilbert gen. et sp. nov. urn:1sid:zoobank.org:act:02DFAF48-FC8F-45CE-BE52-FB1DD05626DF

Fig. 16

\section{Diagnosis}

In general shape, T. rubercella is similar to T. negrosensis but is distinctly different in color. T. rubercella presents a bright yellow pronotum followed by red band running on the basal parts of the tegmen and scutellum when T. negrosensis in dorsal view presents a dirty yellow pronotum followed by a light orange scutelum framed by yellow patches sprawling on the clavus of the tegmen.

\section{Etymology}

This species has a distinct red coloration in the network of cells at the apex of tegmen. The species name is a combination of two latin words "ruber" and "cella" respectively meaning red and cell. The name is placed in apposition.

\section{Material examined}

\section{Holotype}

PHILIPPINES • 7 ; "Philippines, Negros volcan Canlaon, forêt, 10²5'29" N, 12305.36" E"; "Muséum Paris, 1050 m; 28 Oct. 2010; D. Ouvrard Rec", "Muséum Paris, MNHN (EH) 23638”, "séquençage par Elorde Crispolon C-00074"; MNHN. 


\section{Paratypes}

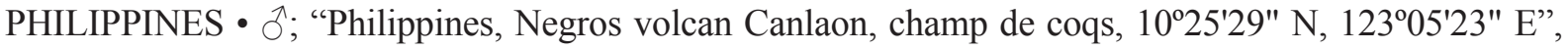
"Muséum Paris, Piège lumineux 932 m; 28 Oct. 2010; A. Soulier-Perkins rec.", "Muséum Paris,

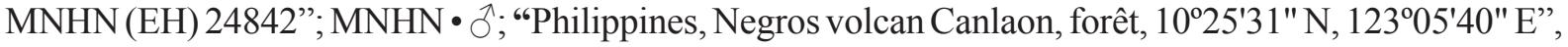
"Muséum Paris, Piège lumineux 1098 m; 29 Oct. 2010; A. Soulier-Perkins rec.", "Muséum Paris, MNHN
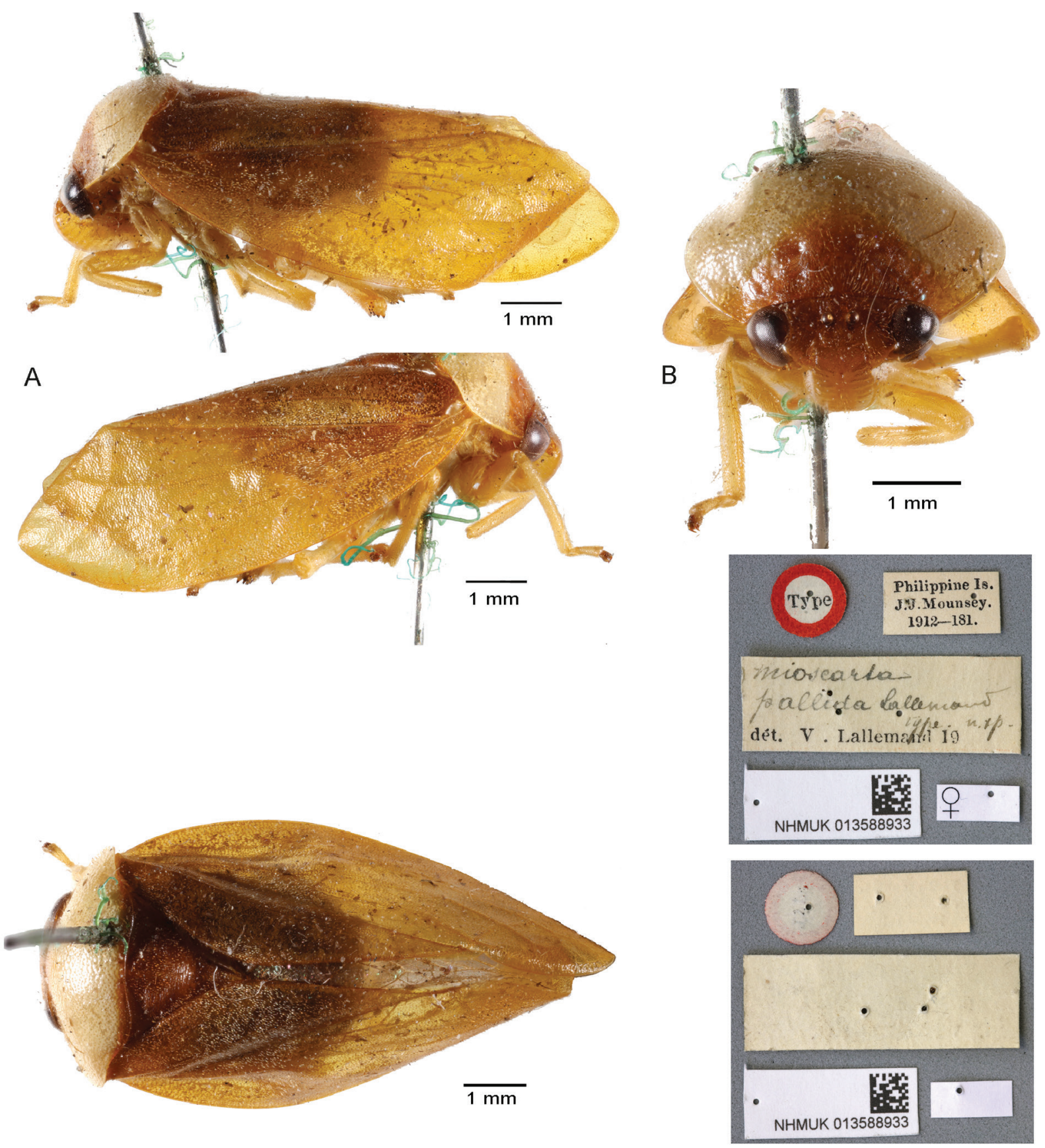

C

D

Fig. 15. Trigonoschema pallida (Lallemand, 1927) comb. nov., holotype (NHMUK 013588933), habitus. A. Lateral view. B. Frontal view. C. Dorsal view. D. Labels. 
CRISPOLON E. Jr. S. et al., New genus and new species of spittlebugs from the Philippines

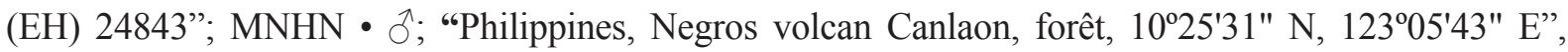
"Muséum Paris, 1098 m; 30. Oct. 2010; D. Ouvrard Rec", "Muséum Paris, MNHN (EH) 24844"; MNHN

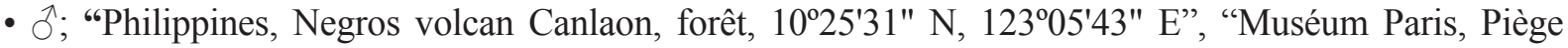
lumineux 1098 m; 30 Oct. 2010; A. Soulier-Perkins rec.", "UPLBMNH HEM-05058"; UPLBMNH.

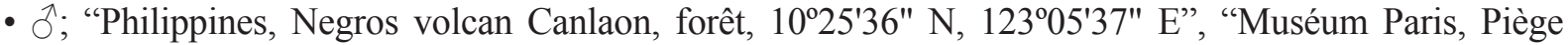

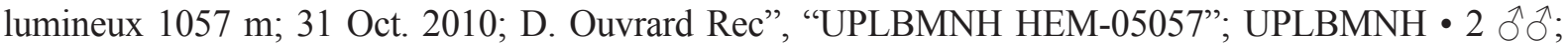
"Philippines, Negros volcan Canlaon, forêt, $10^{\circ} 25^{\prime} 36^{\prime \prime} \mathrm{N}, 123^{\circ} 05^{\prime} 37^{\prime}$ E", "Muséum Paris, Piège lumineux 1057 m; 31 Oct. 2010; A. Soulier-Perkins rec.", "Muséum Paris, MNHN (EH) 24845", "séquençage par Elorde Crispolon C-00075", "Muséum Paris, MNHN (EH) 24846"; MNHN • O’; "Philippines, Negros volcan Canlaon, forêt, 10²5'35" N, 12305'51" E"; "Muséum Paris, 1117 m; 2 Nov. 2010; D. Ouvrard rec.", "Muséum Paris, MNHN (EH) 23640"; MNHN.

\section{Description}

Body LENGTH. 9.5-10.5 mm (tegmina included), width $4.5 \mathrm{~mm}$.

HEAD (Figs 1, 16B). In dorsal view, ocelli small, distance between eyes 9 times ocellus diameter (Figs 1, 16B). Distance between ocellus and compound eye less than 4 times ocellus diameter (Figs 1, 16B). Distance between ocelli 1.5 ocellus diameter (Figs 1, 16B). Eyes not prominent, length 1.33 times than wide. Vertex slightly longer than wide. bearing a median longitudinal carina. Frons without carina. Postclypeus with dimple below margin of frons and a longitudinal furrow, swollen laterally and slightly ovoid shape in frontal view, widest part close to frons, not receding, prior to anteclypeus where it bends forming obtuse angle in lateral view (Figs 1B, 16A). Rostrum long, reaching but not surpassing mesocoxae.

Thorax (Figs 2B, 16A-C). In dorsal view, pronotum with anterior deep concavities on each side, much clearly marked in female, longitudinal median carina absent. Anterior margin of pronotum as wide as posterior margin of head including eyes, anterolateral margins curved, posterior margin grooved, postero-lateral margins slightly concave, slightly longer than anterolateral margins, humeral angle rounded. In lateral view, pronotum angle around $45^{\circ}$ (Figs 2B, 16A). Scutellum as long as wide with large median dimple (Fig. 16C).

TEGMEN (Fig. 16A-C). R bifurcates on apical half, M bifurcate on basal third, apical reticulation not well developed without concave apical cells. Posterior wing (Fig. 3A). Rp separating from SC+Ra nearly at midlength, $\mathrm{M}$ reaches ambient vein, Cua and Cup fused at base and $\mathrm{m}$-cu links $\mathrm{M}$ to Cua before Cua bifurcation, 7 longitudinal veins and 5 apical cells between $\mathrm{SC}+\mathrm{Ra}$ and $\mathrm{Cup}$, angular protrusion of costal margin near its base present. Metafemur with apical spine in inner margin and metatibiae bearing 1 lateral spine.

MaLe terminalia. In lateral view, posterior margin of pygofer (Fig. 16E) convex in middle with slight concavity toward ventral margin. Subgenital plates (Fig. 16F) with equal length relative to height of pygofer with fine appendage shorter than main plate directed posteriorly not forming acute angle with main plate, dorsal and ventral margin of main plate regularly curved. Sterno-lateral plates present, triangular shaped. Intermediate plates present, roughly boomerang-shaped linking internal sides of lateral plate and subgenital plate. Paramere (Fig. 16G) globose, dorsal margin convex then curving regularly and finishing with a sharp spine pointed dorsally, lateral margin with slightly and rounded, ventral margin roughly straight with subapical part angled largely protruding posteriorly then finishing with two sharp spines pointing postero-ventrally. Aedeagus (Fig. 16H) with dorsal margin making a right angle at its base, straight on a small portion before curving up regularly, apical part bending posteriorly and straight, apical extension pointing dorsally, posterior protrusion thick and beak-shaped, postero-dorsal protrusion absent. 


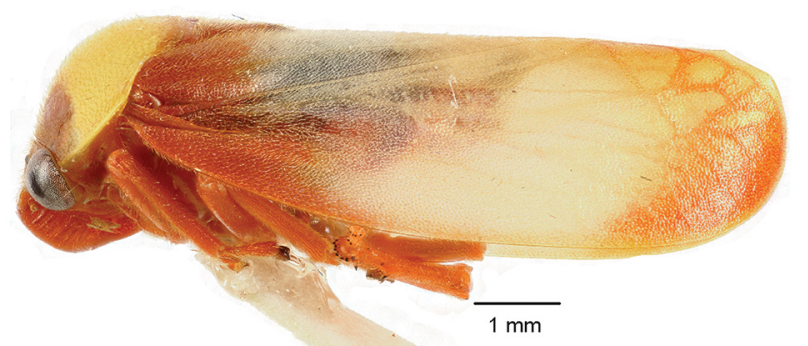

A

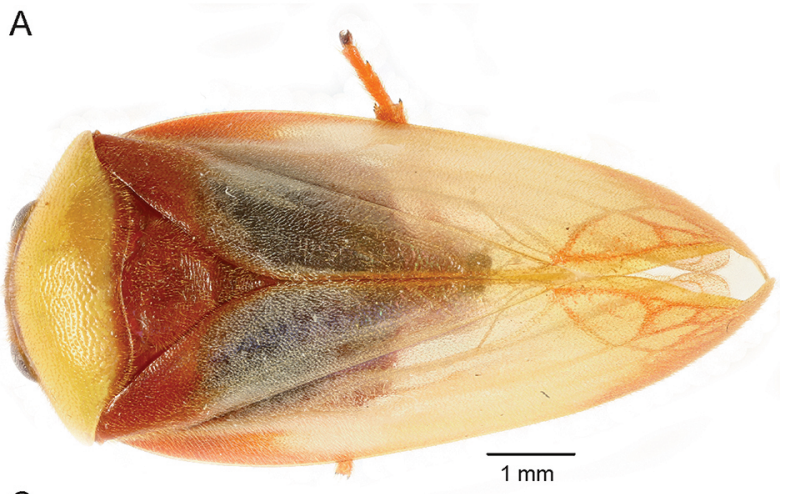

C
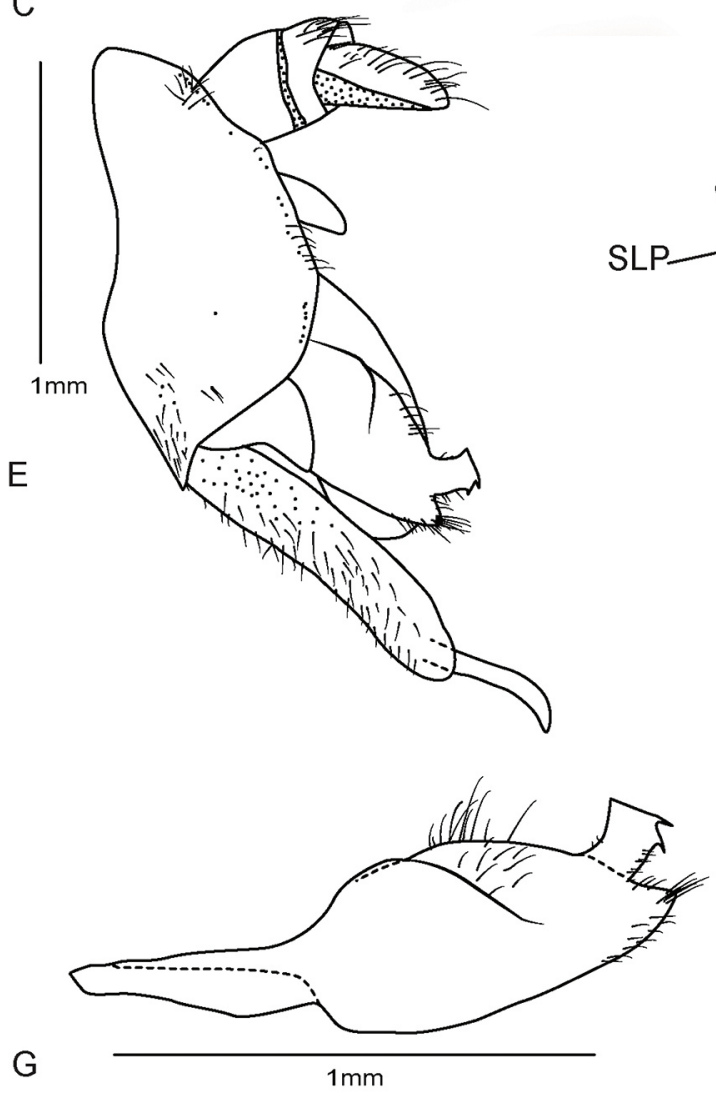

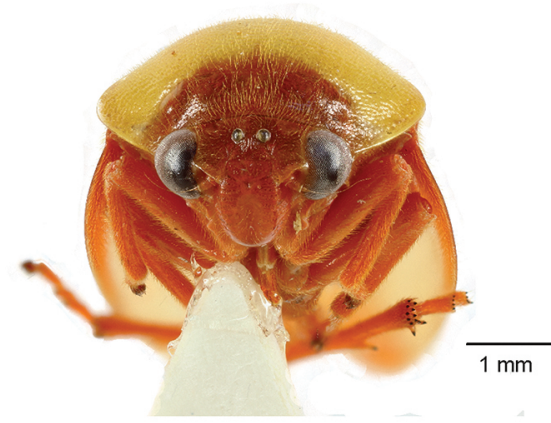

B

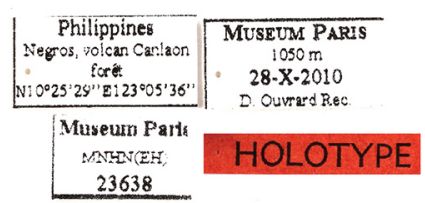

D
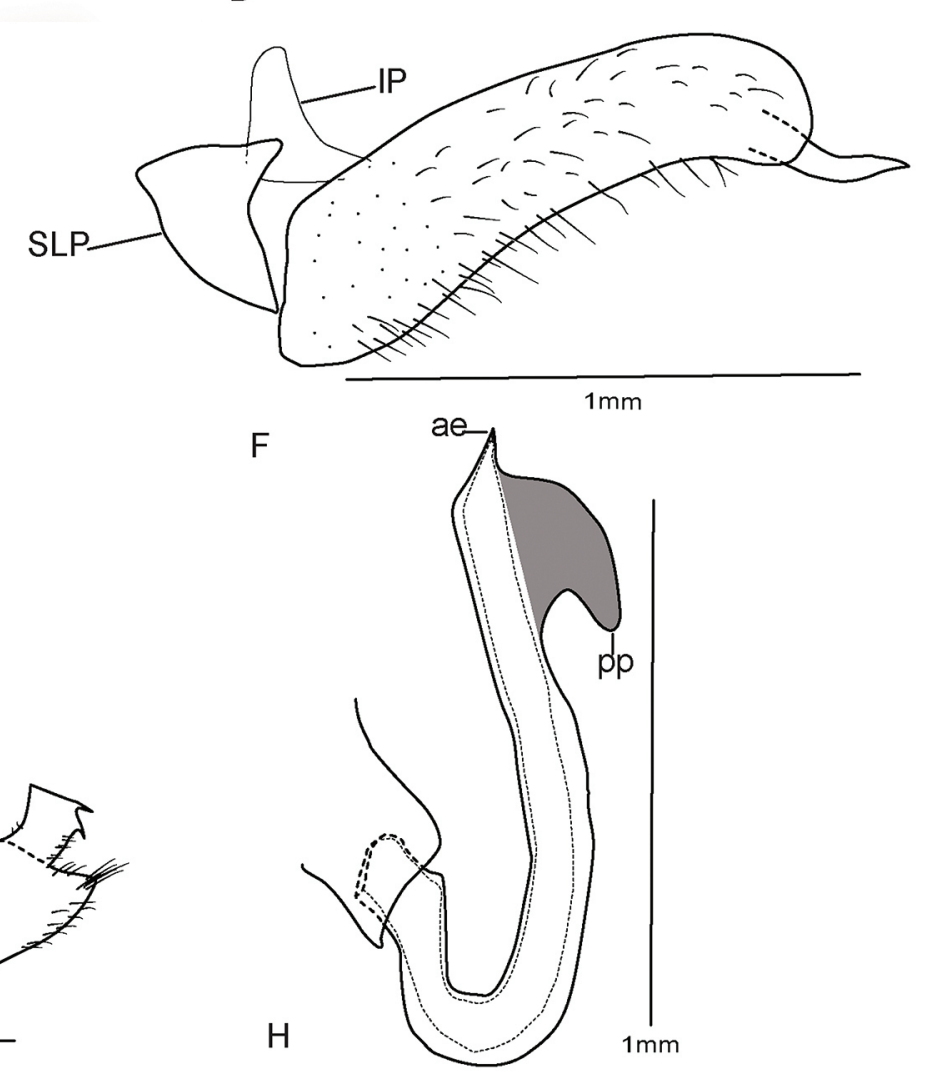

Fig. 16. Trigonoschema rubercella Crispolon \& Guilbert gen. et sp. nov. Holotype. Habitus and $\lesssim$ terminalia in lateral view. A. Lateral view. B. Frontal view. C. Dorsal view. D. Labels. E. Terminalia. F. Sterno-lateral, intermediate and subgenital plates. G. Paramere. H. Aedeagus. 
Color. Head, legs, abdomen, scutellum and anterior part of pronotum red, rest of pronotum yellow, rostrum red, antennal scape and pedicel reddish orange, flagellum yellowish. Tegmen translucid yellow, basal and apical third including network of cells red.

\section{Type Locality}

Philippines: Visayas, Negros Occidental, Mount Kanlaon.

\section{Distribution}

Philippines: Visayas, Negros Island.

\section{Molecular Phylogeny}

Results of the Bayesian 50\% consensus tree and ML analyses with likelihood score of -13870.493 are shown in Figures 17 and 18 respectively. The resulting topologies are not similar with respect to the placement of Jacobsoniella and Wawi. In the Bayesian topology (Fig. 17), Jacobsoniella is recovered within a clade containing Eoscarta, Mioscarta, Poeciloterpa and Trigonoschema gen. nov. clades, each

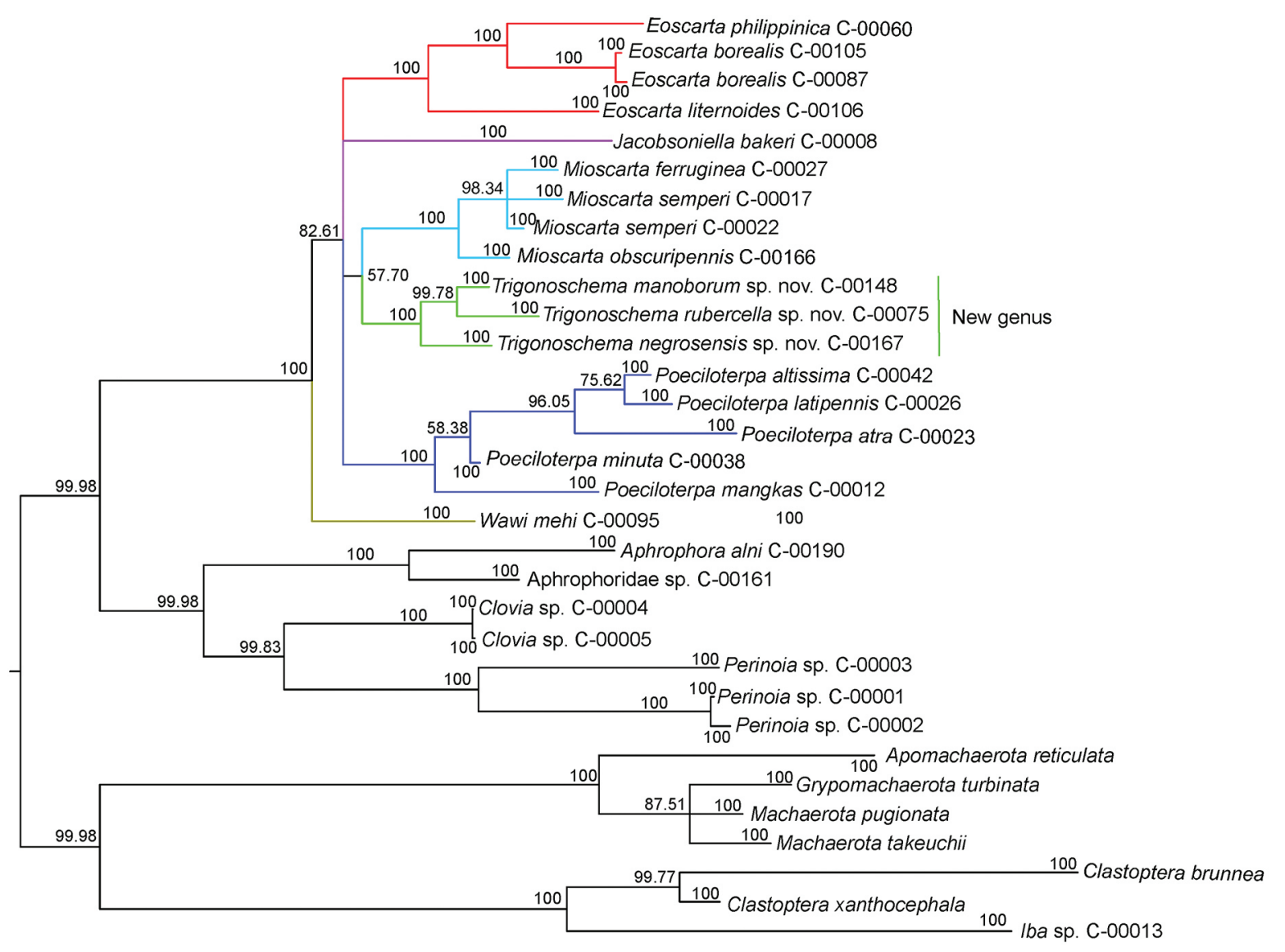

Fig. 17. Bayesian $50 \%$ consensus tree based on partitioned analysis of combined sequences Histone $3+$ CO1 $+18 \mathrm{~S}+28 \mathrm{~S}$ for Eoscarta Breddin, 1902, Jacobsoniella Melichar, 1914, Mioscarta Breddin, 1901, Poeciloterpa Stål, 1870, Trigonoschema Crispolon \& Soulier-Perkins, gen. nov. and Wawi SoulierPerkins \& Le Cesne, 2016 species with Clastopteridae, Machaerotidae and Aphrophoridae as outgroup. Numbers indicated at each resolved nodes are posterior probability values presented in \%. Branches are colored according to genus. 
supported by a probability value $(\mathrm{PPv})$ of $100 \%$. This clade (Eoscarta + Mioscarta + Poeciloterpa + Trigonoschema + Jacobsoniella) is supported PPv of $82.61 \%$ and Wawi appears as sister group to it. However, the clade containing Wawi plus the five other genera is supported with a high PPv of $100 \%$. In the ML analysis (Fig. 18), Poeciloterpa and Trigonoschema gen. nov. are sister groups with a ML bootstrap value (MLBv) of 85.2/21\% (SH-aLRT support/ standard bootstrap support). Then Mioscarta appears as sister group of (Eoscarta + Wawi + (Poeciloterpa + Trigonoschema)). Jacobsoniella is recovered basally as sister of the other ingroup taxa and all together the ingroup clade is supported with a MLBv of $100 / 100 \%$ branch support.

Several key aspects of both topologies are similar. Regardless of the relationships of Eoscarta, Mioscarta, Poeciloterpa, Trigonoschema, Jacobsoniella and Wawi in both topologies the monophyly of each genus is well supported with a PPv of $100 \%$ and MLBv of $98.40 / 91 \%, 97.3 / 96 \%, 99.7 / 99 \%$ and $98.8 / 77 \%$ respectively. In both trees the ingroups are well supported with a PPv of $100 \%$ and MLBv of $100 / 100 \%$.

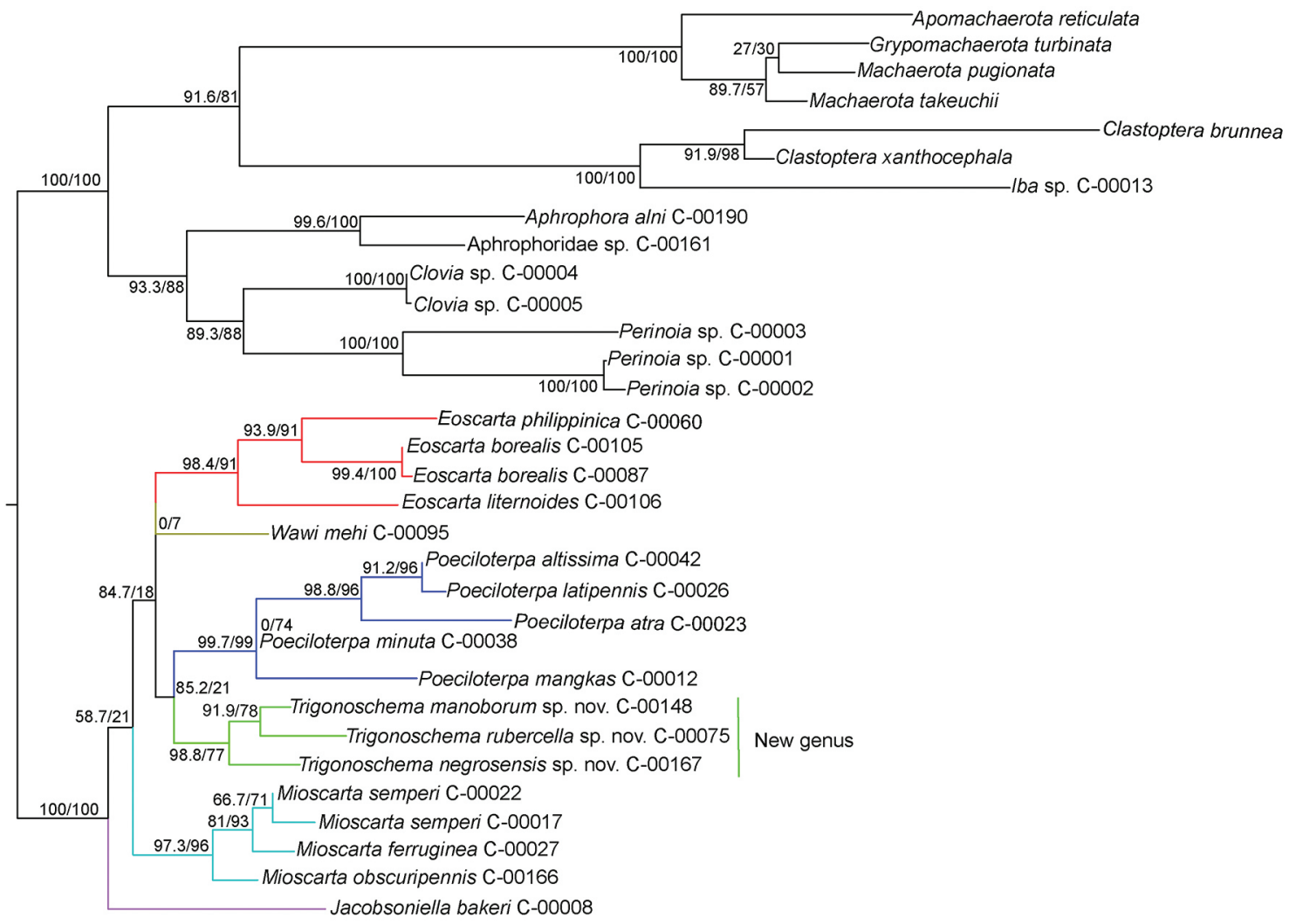

Fig. 18. Maximum likelihood topology (likelihood score of -13870.493) for Eoscarta Breddin, 1902, Jacobsoniella Melichar, 1914, Mioscarta Breddin, 1901, Poeciloterpa Stål, 1870, Trigonoschema Crispolon \& Soulier-Perkins, gen. nov. and Wawi Soulier-Perkins \& Le Cesne, 2016 species with Clastopteridae, Machaerotidae and Aphrophoridae as outgroup based on analyses of combined sequences (CO1, Histone 3, 18S, and 28S). Support statistics from a ML bootstrap value (SH-aLRT support \% / standard bootstrap support \%) are indicated at each resolved node. Branches are colored according to genus. 


\section{Discussion}

\section{Distribution, Biology and Ecology}

Eight species currently belonging to the genus Mioscarta (Fig. 19) and four species of the new genus Trigonoschema (Fig. 20) are known from the Philippines. Of the four species of Trigonoschema gen. nov., only T. pallida cannot be placed precisely since the locality provided by Lallemand (1927) was "Philippine Islands".

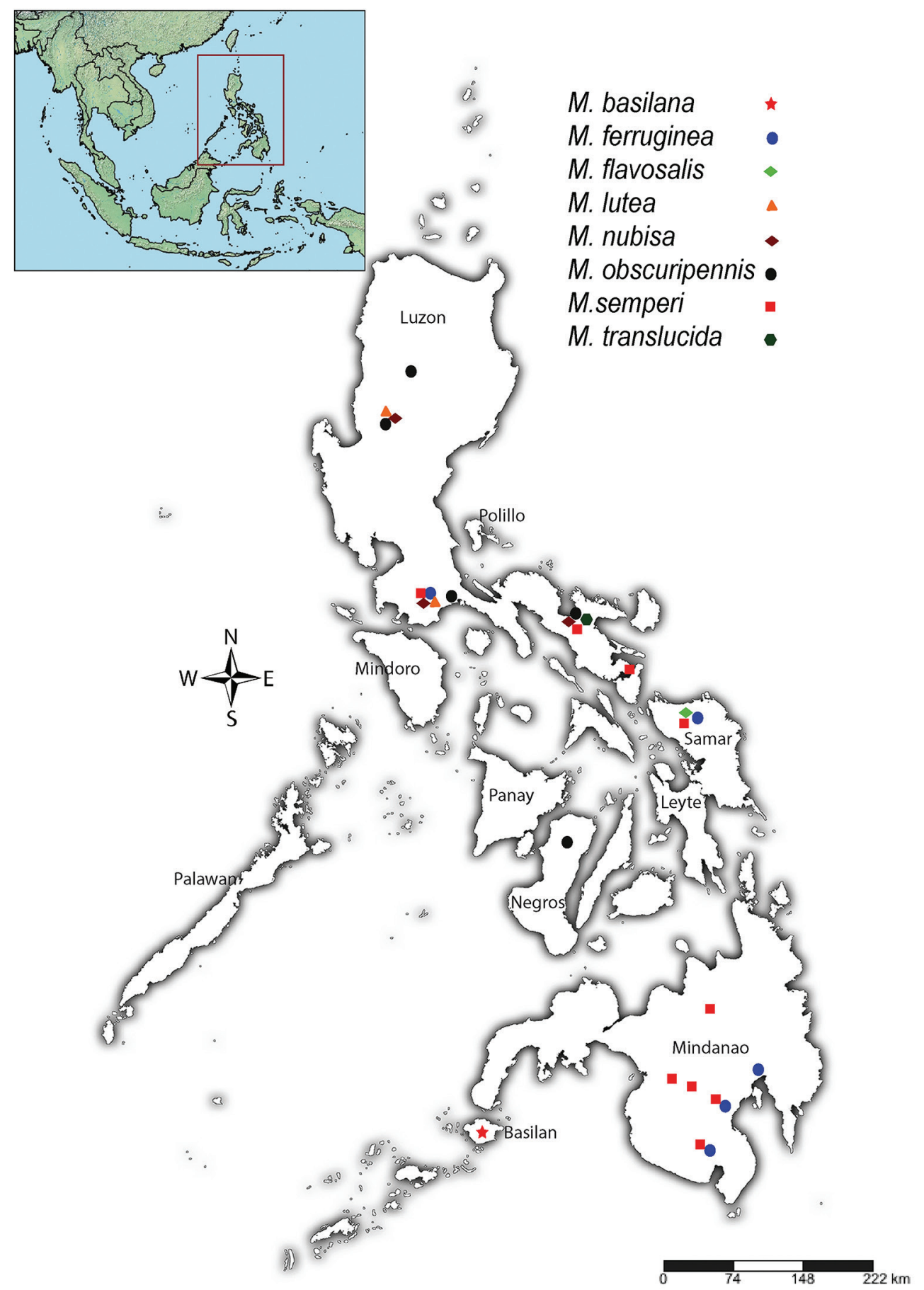

Fig. 19. Philippines Mioscarta Breddin, 1901 distribution map. 
Some specimens were collected using a light trap, thus very little information on their natural history is available to date. Such positive phototaxy was also reported by Soulier-Perkins \& Kunz (2012). Their host-plants remain unknown, even if, some species of both genera were observed alighting on the leaves of different plants of secondary and primary forests. This observation is not enough to conclude the insect's hostplant. Direct observations therefore remain difficult. With the development of next generation sequencing it is possible now to identify plants using their barcoding even when in small

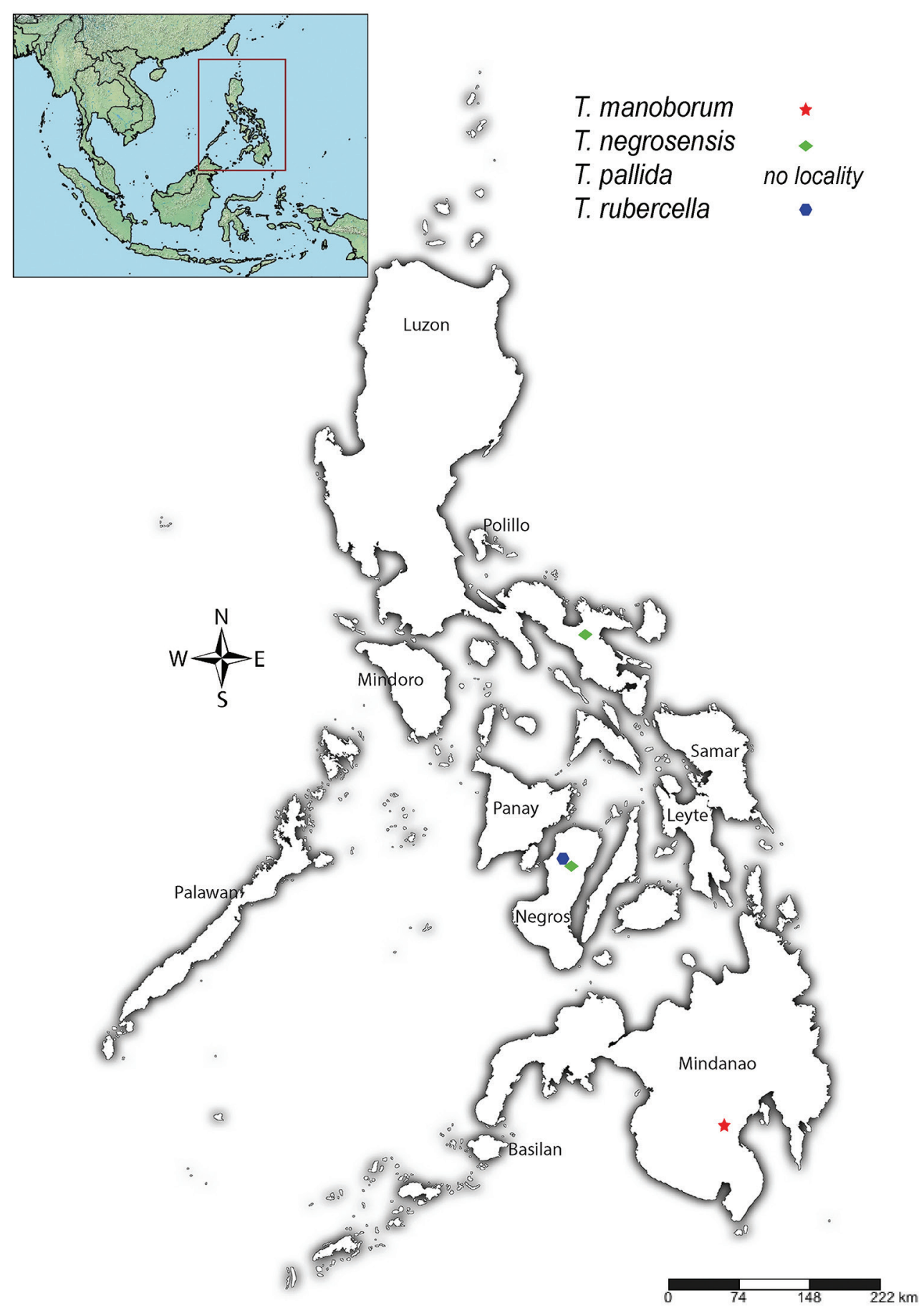

Fig. 20. Trigonoschema Crispolon \& Soulier-Perkins gen. nov. distribution map. 
CRISPOLON E. Jr. S. et al., New genus and new species of spittlebugs from the Philippines

quantity. It is most likely that in a near future, when analysing the content of the insect guts we should find enough genetic material belonging to the ingested host plants that would allow their identification.

\section{Placement of Trigonoschema gen. nov. in the classification}

According to Liang \& Webb (2002) the tribe Rhinaulacini is characterized by a relatively broad head with non-globose eyes and a concave posterior margin of pronotum. It is also usually mentioned that a central longitudinal concave postclypeus and a hind tibia bearing a single strong lateral spine (Lallemand 1949, Liang \& Webb 2002). However, the most obvious character that can separate Rhinaulacini from other Cercopidae is the presence of a sterno-lateral plate between the pygofer and subgenital plate (Liang \& Webb 2002). This sterno-lateral plate is observed in all new described taxa belonging to Eoscarta (Liang \& Webb 2002), Amberana and Bourgoinrana (Soulier-Perkins \& Kunz, 2012), Euryaulax (Liang et al. 2012), Wawi (Soulier-Perkins \& Le Cesne 2016), Poeciloterpa (Crispolon et al. 2019), and Jacobsoniella (based on a specimen we identified), and here in all new Mioscarta and Trigonoschema gen. nov. We are therefore certain in our placement of the new genus Trigonoschema in Rhinaulacini.

Liang \& Webb (2002) formally placed the Eoscartini as a junior synonym of Rhinaulacini without placing the genera in any of the existing subtribes due to the need of further studies on their male terminalia. They emphasized the difficulties in identifying genera and species in this group, separations mainly based on small differences in male genitalia. Trigonoschema gen. nov. possesses characters that allow its clear identification from the other Rhinaulacini genera found in the Philippines, specifically Mioscarta and Poeciloterpa. It appears as well close to those genera, which belong to the Poeciloterpina subtribe. But we have decided for now to follow Liang and Webb's example and leave Trigonoschema gen. nov. as incertae sedis in the Rhinaulacini. The phylogeny shows that Trigonoschema gen. nov. is a distinct clade from Mioscarta. Although the sample is reduced to only six genera with a partial representation for each of them, both resulting topologies (ML and BI) show a well-supported monophyly of Eoscarta, Mioscarta, Poeciloterpa, and Trigonoschema gen. nov., revealing Mioscarta and Trigonoschema gen. nov. as two distinct genera. This justifies the description of the new genus Trigonoschema gen. nov. It is also clear from the differences in topologies between ML and BI, it would be unwise to make assumptions on genera relationships here. All the ingroup genera selected, except Jacobsoniella, belong to the Rhinaulacini and the ingroup support in both analyses is strong but no hasty conclusion should be made on placing Jacobsoniella in the Rhinaulacini or even believing this tribe to be monophyletic. Such hypotheses could only be tested with a larger sampling of Cercopinae genera.

Using the $50 \%$ majority rule topology highlighted the need of a wider analysis including the rest of Cercopidae to reconsider the family's relationships and to build up more robust phylogenetic hypotheses for the family. It is also congruent with the ML analysis, as the relationships between the targeted genera are not well established.

\section{Acknowledgements}

We would like to thank the following persons: Mr Christian Schmidt (SMTD) for lending us type material, Mr Laurent Fauvre (MNHN) Mr Przemysław D. Szymroszczyk (MIZ) and Mr Mick Webb (NHM) for taking most of the photographs presented here, Dr Rhodora Manceras for allowing the first author to join her team for the expedition on Mt. Apo (Mindanao) and Drs Francisco Gil Garcia, Josephine Migalbin, Purificacion Cahatian and Evelyn Esteban (USM) for their support to the first author. Work could not have been done without access to the material kept in the collections of the MNHN and MNH-UPLB and we are in debt to the Local Government Unit of La Carlota for allowing us to conduct our research. We are also grateful to the reviewers for taking their time in improving this paper. Lastly, we thank the CHED-PhilFrance scholarship program for providing the first author the scholarship. 


\section{References}

Astrin J.J. \& Stüben P.E. 2008. Phylogeny in cryptic weevils: molecules, morphology and new genera of western Palaearctic Cryptorhynchinae (Coleoptera: Curculionidae). Invertebrate Systematics 22 (5): 503-522. https://doi.org/10.1071/IS07057

Bartlett C.R., Deitz L.L., Dmitriev D.A., Sanborn A.F., Soulier-Perkins A. \& Wallace M.S. 2018. Chapter 19: The diversity of the true hoppers (Hemiptera: Auchenorrhyncha). In: Foottit R.G. \& Adler P.H. (eds), Insect Biodiversity: Science and Society. II. John Wiley \& Sons Ltd, Chichester: 501-590.

Carvalho G.S. \& Webb M.D. 2005. Cercopid Spittlebugs of new world (Hemiptera: Auchenorrhyncha: Cercopidae). Pensoft Series Faunastica I: 49: 1-271.

Chen X. \& Liang A.-P. 2015. Identification of a self-regulatory pheromone system that controls nymph aggregation behavior of rice spittlebug Callitettix versicolor. Frontiers in Zoology (2015) 12: 10. https://doi.org/10.1186/s12983-015-0102-4

Colgan D.J., McLauchlan A., Wilson G.D.F., Livingston S.P., Edgecombe G.D., Macaranas J., Cassis G. \& Gray M.R. 1998. Histone H3 and U2 snRNA DNA sequences and arthropod molecular evolution. Australian Journal of Zoology 46: 419-437. https://doi.org/10.1071/ZO98048

Crispolon E. S., Yap S. A. \& Soulier-Perkins A. 2019. Revision of the endemic Philippine Poeciloterpa Stål (Hemiptera: Cercopidae) with description of four new species. Zootaxa 4608 (2): 291-328. https://doi.org/10.11646/zootaxa.4608.2.6

Cryan J.R., Wiegmann B.M., Deitz L.L. \& Dietrich C.H. 2000. Phylogeny of the treehoppers (Insecta: Hemiptera: Membracidae): evidence from two nuclear genes. Molecular Phylogenetics and Evolution 17: 317-334. https://doi.org/10.1006/mpev.2000.0832

Deitz L.L., Thompson V., Rakitov R.A., Dietrich C.H., Cryan J.R. \& Alvarez P.A. 2008. DrMetcalf: a resource on cicadas, leafhoppers, planthoppers, spittlebugs, and treehoppers. Available from:

https://www.lib.ncsu.edu/specialcollections/digital/metcalf/auchenorrhyncha.html (accessed 29 June 2015).

Dietrich C.H., Rakitov R.A., Holmes J.L. \& Black W.C. 2001. Phylogeny of the major lineages of Membracoidea (Insecta: Hemiptera: Cicadomorpha) based on 28S rDNA sequences. Molecular Phylogenetics and Evolution 18: 293-305. https://doi.org/10.1006/mpev.2000.0873

Distant W. L. 1908. Rhynchota Malayana. Part 1. Records of the Indian Museum. Calcutta 2: 127-151.

Guindon S., Dufayard J. F., Lefort V., Anisimova M., Hordijk W. \& Gascuel O. 2010. New algorithms and methods to estimate maximum-likelihood phylogenies: assessing the performance of PhyML 3.0. Systematic biology 59 (3): 307-321. https://doi.org/10.1093/sysbio/syq010

Hillis D.M. \& Dixon M.T. 1991. Ribosomal DNA: molecular evolution and phylogenetic inference. The Quarterly Review of Biology 66: 411-453. https://doi.org/10.1086/417338

Hoang D.T., Chernomor O., von Haeseler A., Minh B.Q. \& Vinh L.S. 2018. UFBoot2: Improving the ultrafast bootstrap approximation. Molecular Biology and Evolution 35: 518-522.

https://doi.org/10.1093/molbev/msx281

Holmann F. \& Peck D.C. 2002. Economic Damage Caused by Spittlebugs (Homoptera: Cercopidae) in Colombia: A First Approximation of Impact on Animal Production in Brachiaria decumbens Pastures. Neotropical Entomology 31 (2): 275-284. https://doi.org/10.1590/S1519-566X2002000200016

Huelsenbeck J.P. \& Ronquist F. 2001. MrBayes: Bayesian inference of phylogeny. Bioinformatics 17: 754-755. https://doi.org/10.1093/bioinformatics/17.8.754 
CRISPOLON E. Jr. S. et al., New genus and new species of spittlebugs from the Philippines

Huelsenbeck J.P. \& Imennov N.S. 2002. Geographic origin of human mitochondrial DNA: accommodating phylogenetic uncertainty and model comparison. Systematic Biology 51: 673-688. https://doi.org/10.1080/106351502753475934

Huelsenbeck J.P., Larget B., Miller R.E. \& Ronquist F. 2002. Potential applications and pitfalls of Bayesian inference phylogeny. Systematic Biology 51: 673-688. https://doi.org/10.1080/10635150290102366

Jacobi A. 1905. Studien über die Homopterenfamilie der Cercopiden. I. Gattungen des indoaustralischen Faunengebietes. Mitteilungen aus dem Zoologischen Museum in Berlin. Berlin 3: 5-24.

Jacobi A. 1927. Ueber einige Tomaspidinae (Rhynchota, Homoptera) von den Philippinen. Philippine Journal of Science, Manila 32: 549-551.

Katoh K. \& Standley D.M. 2013. MAFFT multiple sequence alignment software version 7: improvements in performance and usability. Molecular Biology and Evolution 30: 772-780.

https://doi.org/10.1093/molbev/mst010

Lallemand V. 1912. Homoptera Fam. Cercopidae. Genera Insectorum. Diriges par P. Wytsman, Bruxelles 143: $1-167$.

Lallemand V. 1927. Descriptions de Cercopides nouveaux provenant de la collections du British Muséum. Transactions of the Entomological Society of London 1: 99-118.

https://doi.org/10.1111/j.1365-2311.1927.tb00063.x

Lallemand V. 1949. Revision des Cercopinae (Hemiptera Homoptera) Première partie. Mémoires de l'Institut Royal des Sciences Naturelles de Belgique Series 2 (32): 1-193.

Lallemand V. \& Synave H. 1961. Revision des Cercopinae (Hemiptera Homoptera) Deuxième partie. Mémoires de l'Institut Royal des Sciences Naturelles de Belgique, Series 2 (66): 1-153.

Lanfear R., Calcott B., Ho S. Y. \& Guindon S. 2012. PartitionFinder: combined selection of partitioning schemes and substitution models for phylogenetic analyses. Molecular Biology and Evolution 29 (6): 1695-1701. https://doi.org/10.1093/molbev/mss020

Lanfear R., Frandsen P. B., Wright A. M., Senfeld T. \& Calcott B. 2016. PartitionFinder 2: new methods for selecting partitioned models of evolution formolecular and morphological phylogenetic analyses. Molecular Biology and Evolution. https://doi.org/10.1093/molbev/msw260

Le Cesne M., Crispolon E. \& Soulier-Perkins A. 2021. Male terminalia of Cercopidae (Hemiptera, Cicadomorpha): towards a consensus terminology. Scientific Reports 11, 10412 (2021). https://doi.org/10.1038/s41598-021-89759-3

Liang A.-P. \& Webb M.D. 2002. New taxa and revisionary notes in Rhinaulacini spittlebugs from southern Asia (Homoptera: Cercopidae). Journal of Natural History 36 (6): 729-756.

https://doi.org/10.1080/00222930110062336

Liang A.-P., Combéfis É. \& Jiang G.-M. 2012. Revision of the Spittlebug Genus Euryaulax Kirkaldy (Hemiptera:Cercopoidea: Cercopidae) from Australia. Annals of the Entomological Society of America 105 (2): 150-164. https://doi.org/10.1603/AN11088

Mello M. L. S., Faustino De Carvalho H. \& Nogueira De Sa L. A. 1996. Topochemistry and structure of the froth of different spittlebug species. Revista Brasileira de Biologia 56 (1): 177-181.

Miller M.A., Pfeiffer W. \& Schwartz T. 2010. Creating the CIPRES Science Gateway for inference of large phylogenetic trees: in Proceedings of the Gateway Computing Environments Workshop (GCE), 14 Nov. 2010, New Orleans, LA: 1-8. https://doi.org/10.1109/GCE.2010.5676129

Minh B.Q., Nguyen M.A. \& von Haeseler A. 2013. Ultrafast approximation for phylogenetic bootstrap. Molecular Biology and Evolution 30: 1188-1195. https://doi.org/10.1093/molbev/mst024 
Nguyen L.T., Schmidt H.A., von Haeseler A. \& Minh B.Q. 2015. IQ-TREE: a fast and effective stochastic algorithm for estimating maximum-likelihood phylogenies. Molecular Biology and Evolution 32: 268-274. https://doi.org/10.1093/molbev/msu300

Paladini A., Ferrari A. \& Carvalho G.S. 2008. Cladistic Analysis of Kanaima Distant, 1909 (Hemiptera, Cercopidae). Zootaxa 1704: 47-63. https://doi.org/10.11646/zootaxa.1704.1.4

Rambaut A. 2016. FigTree v1.4.3: Tree figure drawing tool. http://ree.bio.ed.ac.uk/software/figtree/ [accessed in 4 November 2020].

Ranwez V., Harispe S., Delsuc F. \& Douzery E.J.P. 2011. MACSE: Multiple Alignment of Coding SEquences accounting for frameshifts and stop codons. PLoS One 20116 (9): e22594.

https://doi.org/10.1371/journal.pone.0022594

Ranwez V., Douzery E.J.P., Chantret N. \& Delsuc F. 2018. MACSE v2: Toolkit for the Alignment of Coding Sequences Accounting for Frameshifts and Stop Codons. Molecular Biology and Evolution 35 (10): 2582-2584. https://doi.org/10.1093/molbev/msy159

Ronquist F. \& Huelsenbeck J.P. 2003. MrBayes 3: Bayesian phylogenetic inference under mixed models. Bioinformatics 19: 1572-1574. https://doi.org/10.1093/bioinformatics/btg180

Schmidt E. 1909. Neue und bekannte Gattungen und Arten der Subfamilie Cercopinae Stål des indoaustralischen Faunengebi- etes, ein Beitrag zur Kenntnis der Cercopiden. (Hemiptera-Homoptera). In: Entomologische Zeitung. Vol. 70. Herausgegeben von dem entomologischen Vereine zu Stettin, Stettin: 146-187.

Schmidt E. 1920. Neue Zikaden von den Philippinen, Sumatra und Java. (Rhynchota-Homoptera). In: Entomologische Zeitung. Vol. 81. Herausgegeben von dem entomologischen Vereine zu Stettin, Stettin: 43-56.

Schmidt E. 1925. Fünf neue Zikadenarten (Forts f.). Societas Entomologica. Organ für den internationalen Entomologenverein 40: 35-36.

Soulier-Perkins A. \& Kunz G. 2012. Revision of the malagassy endemic genus Amberana Distant (Hemiptera, Cercopidae) with description of one new genus. Zootaxa 3156: 1-42.

https://doi.org/10.11646/zootaxa.3156.1.1

Soulier-Perkins A. \& Le Cesne M. 2016. A new Papuan cercopid genus (Hemiptera: Cicadomorpha: Cercopidae) and checklist of the species found on the Island of New Guinea. In: Robillard T., Legendre F., Villemant C. \& Leponce M. (eds), Insects of Mount Wilhelm, Papua New Guinea. Muséum national d'Histoire naturelle, Paris: 117-128.

Soulier-Perkins A. 2020. COOL - Cercopoidea Organised On Line. Available from: http://hemiptera-databases.org/cool/ [accessed in September 2020].

Stål C. 1870. Hemiptera insularum Philippinarum. Bidrag till Philippinska öarnes Hemipter-fauna. Ofversigt af Kongliga Svenska Vetenskaps-Akademiens Förhandlingar. Stockholm 27: 607-776. https://doi.org/10.5962/bhl.title.61898

Su T., He B., Li K. \& Liang A.P. 2018. Comparative analysis of the mitochondrial genomes of oriental spittlebug tribe Cosmoscartini: insights into the relationships among closely related taxa. BMC Genomics (2018) 19: 961. https://doi.org/10.1186/s12864-018-5365-7

Thompson V. 2004. Associative nitrogen fixation, C4 photosynthesis, and the evolution of spittlebugs (Hemiptera: Cercopidae) as major pests of Neotropical sugarcane and forage grasses. Bulletin of Entomological Research 94: 189-200. https://doi.org/10.1079/BER2004293 
CRISPOLON E. Jr. S. et al., New genus and new species of spittlebugs from the Philippines

Thompson V., Halbert S. E. \& Rothschild M. 2020. A new species of the spittlebug genus Clastoptera Germar (Hemiptera: Cercopoidea: Clastopteridae) on Florida oaks. Insecta Mundi 0796: 1-16.

Vallejo Jr. B. 2014. The Biogeography of Luzon Island. In: Telnov, D. (Ed.), Biodiversity, Biogeography and Nature Conservation in Wallacea and New Guinea. Vol. 2. The Entomological Society of Latvia, Rîga: 47-59. Available from:

https://www.researchgate.net/publication/267212078_The_Biogeography_of_Luzon_Island

Walker F. 1851. List of the specimens of Homopterous insects in the collection of the British Museum 3: 637-907. https://www.biodiversitylibrary.org/page/9619677

Whiting M.F. 2002. Phylogeny of the holometabolous insect orders based on 18S ribosomal DNA: when bad things happen to good data. In: DeSalle, R., Giribet, G., Wheeler, W. (ed.), Molecular Systematics and Evolution: Theory and Practice, Birkhäuser, Basel: 69-83.https://doi.org/10.1007/978-3-0348-8114-2_5

Whiting M.F., Carpenter J.C., Wheeler Q.D. \& Wheeler W.C. 1997. The Strepsiptera problem: phylogeny of the holometabolous insect orders inferred from $18 \mathrm{~S}$ and $28 \mathrm{~S}$ ribosomal DNA sequences and morphology. Systematic Biology 46: 1-68. https://doi.org/10.1093/sysbio/46.1.1

Zhang D., Gao F., Jakovlić I., Zou H., Zhang J., Li W.X. \& Wang G.T. 2020. PhyloSuite: An integrated and scalable desktop platform for streamlined molecular sequence data management and evolutionary phylogenetics studies. Molecular Ecology Resources 202020 (1): 348-355.

https://doi.org/10.1111/1755-0998.13096

Manuscript received: 11 March 2021

Manuscript accepted: 9 September 2021

Published on: 16 November 2021

Topic editor: Nesrine Akkari

Desk editor: Fariza Sissi

Printed versions of all papers are also deposited in the libraries of the institutes that are members of the EJT consortium: Muséum national d'histoire naturelle, Paris, France; Meise Botanic Garden, Belgium; Royal Museum for Central Africa, Tervuren, Belgium; Royal Belgian Institute of Natural Sciences, Brussels, Belgium; Natural History Museum of Denmark, Copenhagen, Denmark; Naturalis Biodiversity Center, Leiden, the Netherlands; Museo Nacional de Ciencias Naturales-CSIC, Madrid, Spain; Real Jardín Botánico de Madrid CSIC, Spain; Zoological Research Museum Alexander Koenig, Bonn, Germany; National Museum, Prague, Czech Republic. 